Effects of Micro-Ramps on a Shock Wave/Turbulent Boundary Layer Interaction

P.L. Blinde

October 2008 


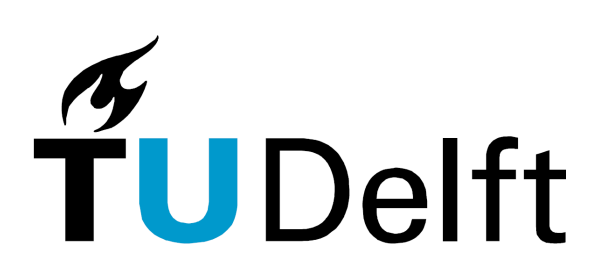

\section{Delft University of Technology}

Copyright (C) Aerospace Engineering, Delft University of Technology All rights reserved. 


\title{
Effects of Micro-Ramps on a Shock Wave/Turbulent Boundary Layer Interaction
}

\author{
Master of Science Thesis \\ For obtaining the degree of Master of Science in Aerospace \\ Engineering at Delft University of Technology
}

P.L. Blinde

October 13, 2008

Faculty of Aerospace Engineering · Delft University of Technology 



\section{DELFT UNIVERSITY OF TECHNOLOGY \\ DEPARTMENT OF AERODYNAMICS}

The undersigned hereby certify that they have read and recommend to the Faculty of Aerospace Engineering for acceptance the thesis entitled "Effects of Micro-Ramps on a Shock Wave/Turbulent Boundary Layer Interaction" by P.L. Blinde in fulfillment of the requirements for the degree of Master of Science.

Dated: October 27, 2008

Supervisors:

Prof.dr. F. Scarano

Dr. ir. B.W. van Oudheusden

R.A. Humble, MEng

Ir. L.J. Souverein 

Shock wave/turbulent boundary layer interactions (SWTBLIs) are a class of fluid dynamic phenomena that are prevalent in many applications, e.g. helicopter blades, supersonic inlets, missile and aircraft after bodies, etc. The interactions are an important source of drag and can cause turbulent and unsteady separation of the boundary layer, leading to increased heat fluxes and fluctuating pressure loads, which can be severe enough to cause structural damage and premature fatigue of aero-structures. By placing vortex generators (VGs) upstream of a SWTBLI, the detrimental effects of the shock induced separation can be diminished. One particular type of VG is the micro-ramp. Because of their wedge-like shape, micro-ramps are very robust and easy to apply compared to most other VGs.

Recently, studies that made use of velocity data in wall-parallel planes have led to renewed insight in the role of variations in the incoming boundary layer on the interaction's phenomenology. For this reason, stereo-particle image velocimetry (stereo-PIV) measurements at two wall-parallel have been performed to investigate the effect of two configurations of micro-ramps, a single row and a staggered array, on an oblique shock reflection on a flat plate at a freestream Mach number of 1.84. The micro-ramps had a height of $20 \%$ of the unperturbed boundary layer thickness and the measurement planes were located 0.1 and 0.6 boundary layer thicknesses away from the wall. Additionally, oil-flow patterns at the surface were obtained.

The micro-ramps were observed to generate individual vortex pair packets downstream of their vertices that on the mean look like longitudinal streamwise vortex pairs. These structures perturb the incoming boundary layer in such a way, that on the mean low-speed regions exist downstream of the vertices and high-speed regions at intermediate locations. Downstream of low-speed regions in the incoming boundary layer the probability of reversed flow occurrence in the interaction region was found to be higher and the subsonic region to be longer in streamwise direction. These and other results have been used to construct a tentative conceptual model of the effect of micro-ramps on the boundary layer's and interaction's topology.

Overall, the probability of the occurrence of reversed flow in the interaction region decreased by $20 \%$ and $30 \%$ downstream of the single row and the staggered configuration respectively. Both configurations of micro-ramps were found to stabilize the shock motion by reducing the length of its motion by about $20 \%$ in the lower measurement plane. Farther away from the wall only the single row configuration was found to be effective, leading to a spanwise averaged reduction of $30 \%$ in the upper measurement plane. Because of all these benefits, it is recommended to further develop micro-ramp control, whereby the conceptual flow model proposed in the present investigation can provide (part of) an initial framework in which this can be done. 
EFFECTS OF MICRO-RAMPS ON A SHOCK WAVE BOUNDARY LAYER INTERACTION 


\section{Acknowledgments}

First of all I would like to thank prof. dr. F. Scarano and dr. ir. B.W. van Oudheusden for their supervision. Furthermore I would like to thank the technical staff, consisting of ing. E. de Keizer, ing. F. Donker Duyvis, N. van Beek and P.J. Duijndam for helping out with the wind tunnel operation and experiment preparation when needed. Special thanks go out to Ray Humble, whose advices and insights were indispensible and without whom the quality of my work and the experience of conducting fluid dynamical research would not have been the same.

Above all, I would like to thank my parents and grandmother, who have supported me throughout my student career. Thanks also go out to my girlfriend Marijke, although -as she would undoubtedly agree- this is not for any fluid dynamical insight. Last but not least, I would like to thank all of my friends.

Paul Blinde

Delft, October 2008 
EFFECTS OF MICRO-RAMPS ON A SHOCK WAVE BOUNDARY LAYER INTERACTION 


\section{Table of Contents}

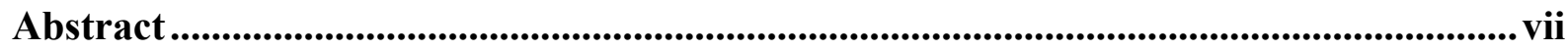

Acknowledgments..................................................................................................................... ix

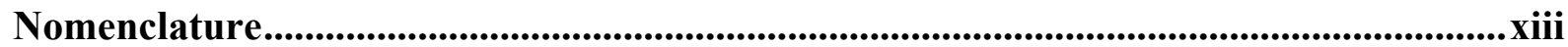

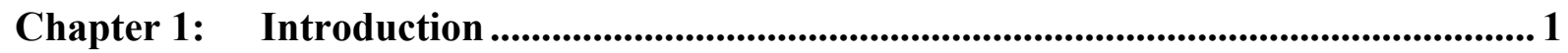

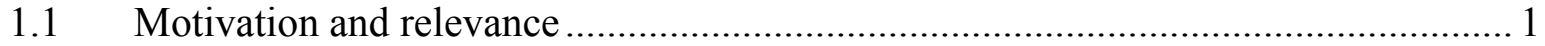

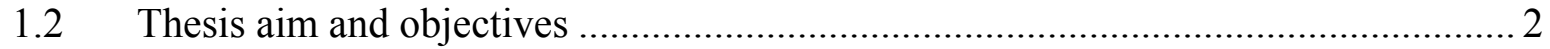

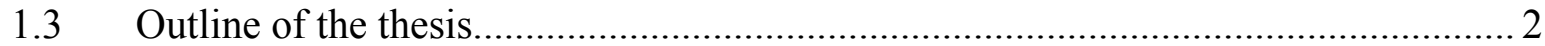

Chapter 2: Background of SWTBLIs and their Control by Micro-Ramps ................... 3

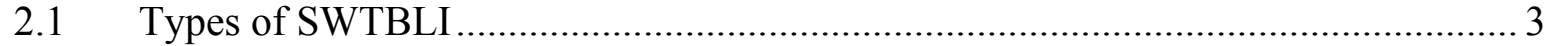

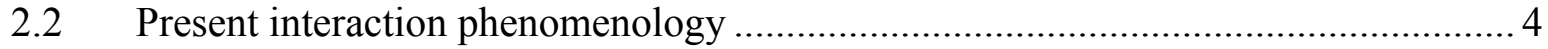

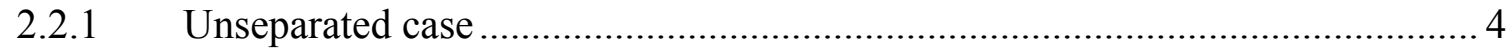

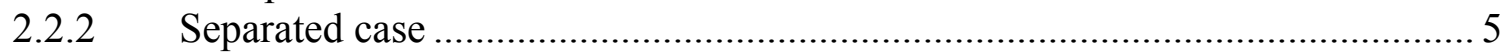

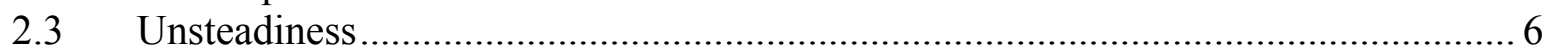

2.4 Source(s) of low-frequency unsteadiness..................................................... 8

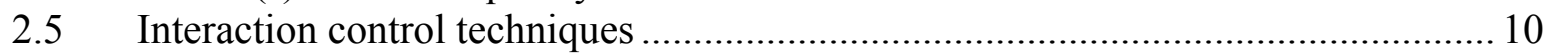

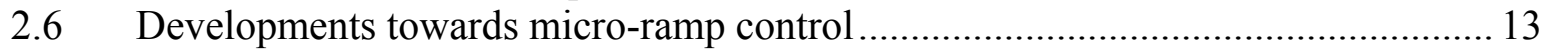

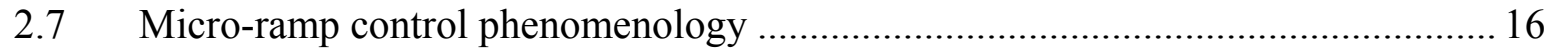

Chapter 3: $\quad$ Experimental Techniques and Arrangement .............................................. 19

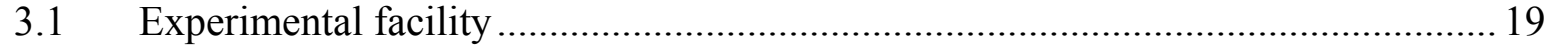

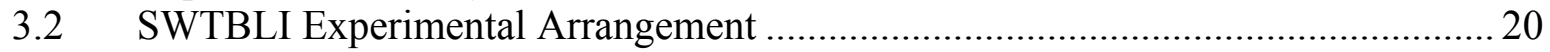

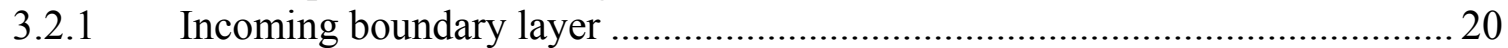

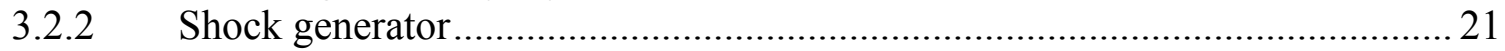

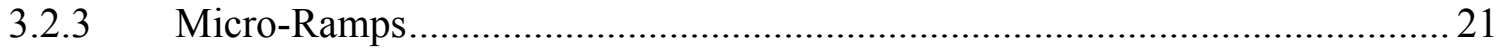

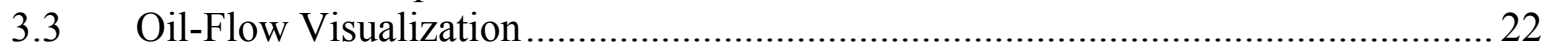

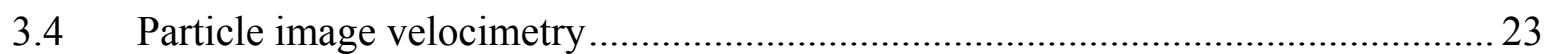

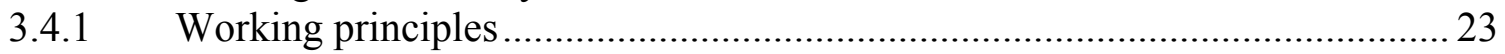

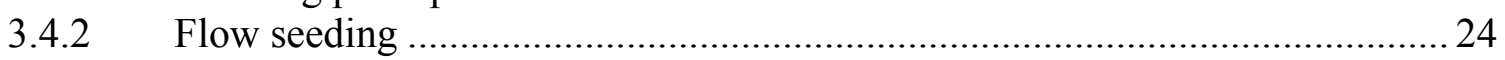

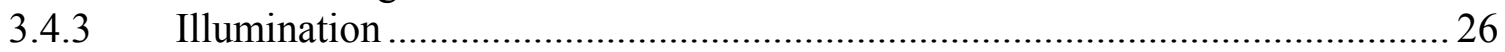

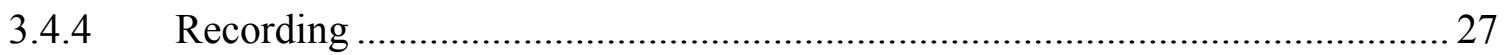

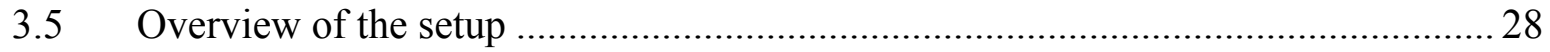

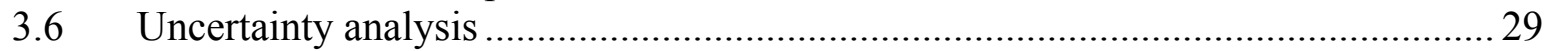

Chapter 4: Oil-Flow Visualization Results and Discussion .........................................33

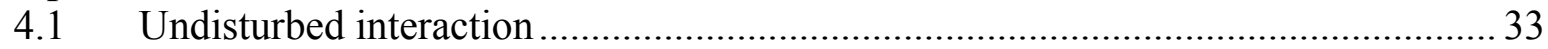

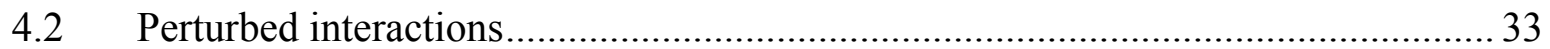

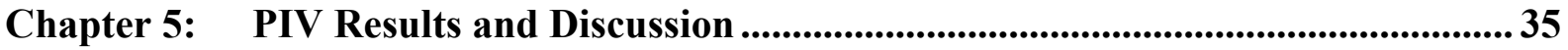

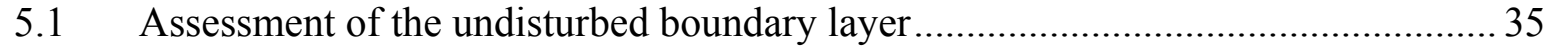

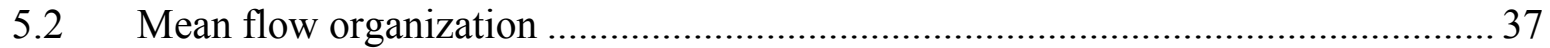

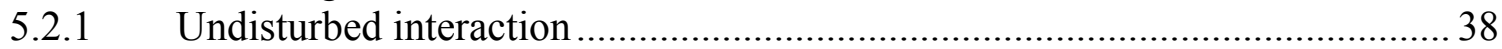

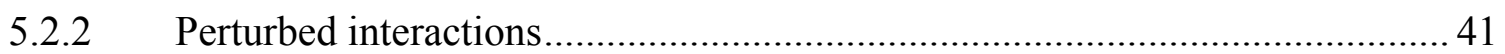




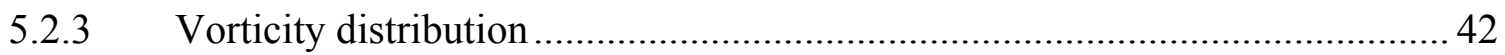

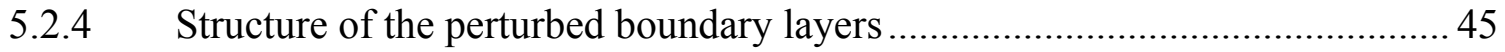

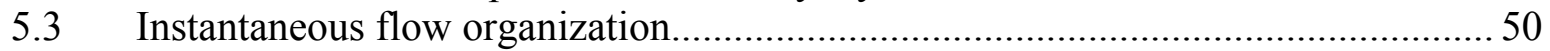

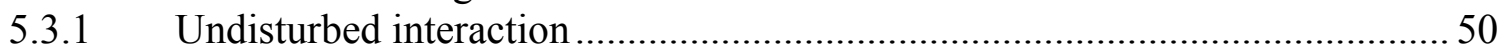

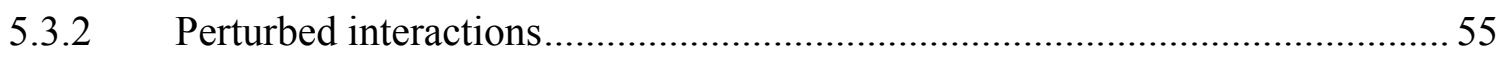

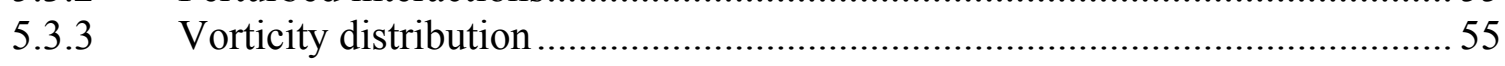

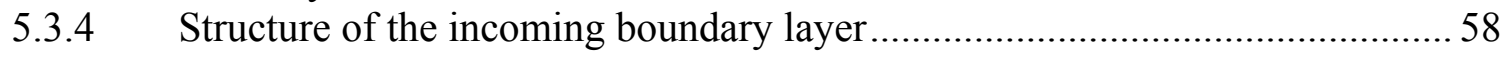

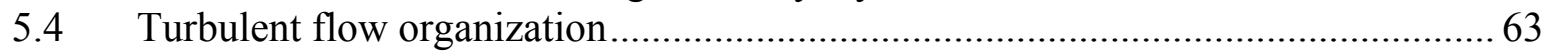

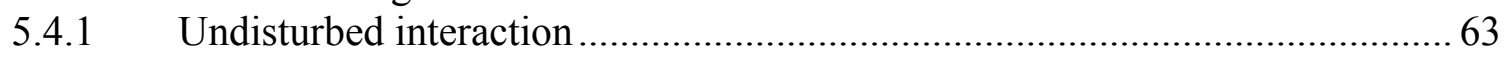

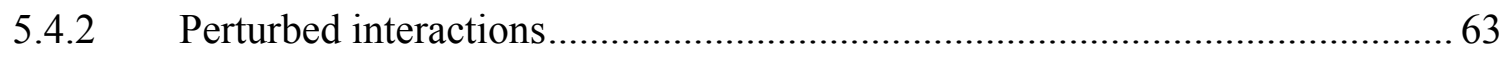

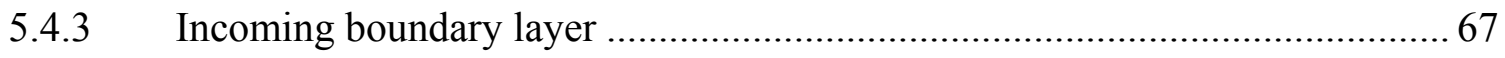

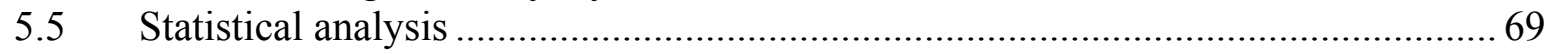

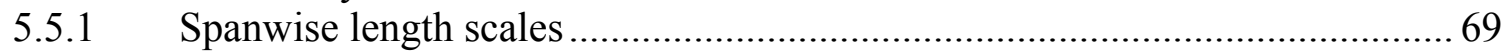

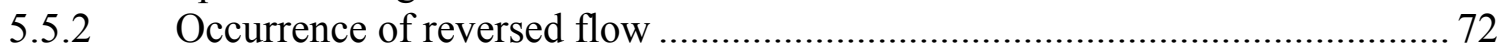

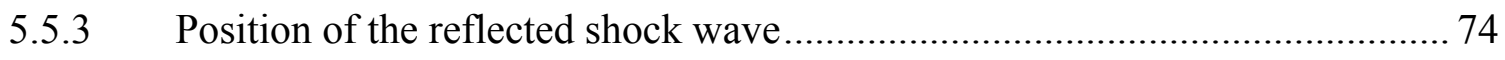

5.5.4 Relation between velocity fluctuations and shock position ........................... 76

Chapter 6: Conceptual Model ................................................................................... 81

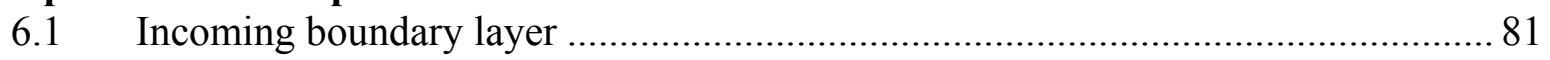

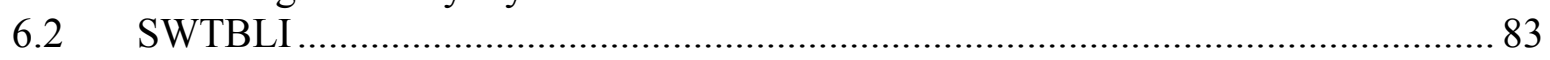

Chapter 7: Conclusions and Recommendations .......................................................... 85

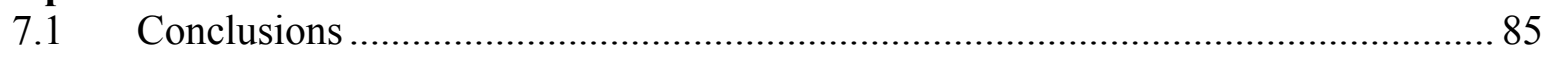

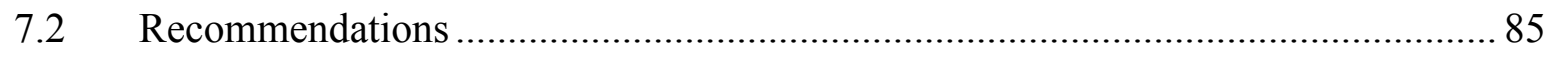

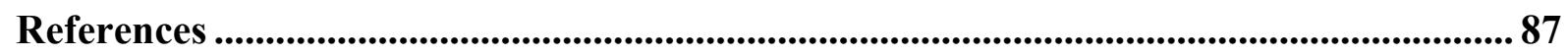




\section{Nomenclature}

D micro-ramp angle of incidence

angle between CCD chip and measurement plane

ratio of specific heats

boundary layer thickness

displacement thickness

root mean square uncertainty

momentum thickness

spatial resolution

representative length

static fluid viscosity

dynamic viscosity

relaxation length

density

standard deviation

fluid time scale

particle relaxation time

nomalized vorticity

speed of sound

micro-ramp chord length

drag coefficient

friction coefficient

particle diameter

distance between to counter-rotating vortices

with a common flow down

focal length

focal number

particle frequency responds

micro-ramp height

distance between calibration plate dots corresponding to $n_{c}$ pixels number of pixels on calibration plate corresponding to $l_{c}$ recovery factor

distance between centre-lines of two adjacent micro-ramps

displacement

streamwise velocity component

friction velocity

wall-normal velocity component

spanwise velocity component

Cartesian coordinate in streamwise direction

mean position of reflected shock proxy

at a given spanwise position

Cartesian coordinate in wall-normal direction

Cartesian coordinate in spanwise direction $\left[{ }^{\mathrm{o}}\right]$

$\left[{ }^{\mathrm{o}}\right]$

$[-]$

[mm]

[mm]

$[-]$

[mm]

[mm/pix.]

[ - ]

$\left[\mathrm{kg} \mathrm{m}^{-1} \mathrm{~s}^{-1}\right]$

$\left[\mathrm{m}^{2} \mathrm{~s}^{-1}\right]$

[mm]

$\left[\mathrm{kg} \mathrm{m}^{-3}\right]$

[- ]

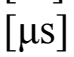

[ $\mu \mathrm{s}]$

[-]

$\left[\mathrm{m} \mathrm{s}^{-1}\right]$

[mm]

[- ]

[-]

[mm]

[mm]

[mm]

[-]

$\left[\mathrm{s}^{-1}\right]$

[mm]

[mm]

[ - ]

[- ]

[mm]

[mm]

$\left[\mathrm{m} \mathrm{s}^{-1}\right]$

$\left[\mathrm{m} \mathrm{s}^{-1}\right]$

$\left[\mathrm{m} \mathrm{s}^{-1}\right]$

$\left[\mathrm{m} \mathrm{s}^{-1}\right]$

[mm]

[mm]

[mm]

[mm]

[mm] 


$\begin{array}{llc}\mathrm{L} & \text { length } & {[\mathrm{mm}]} \\ \mathrm{M} & \text { Mach number } & {[-]} \\ \mathrm{N} & \text { number of recordings } & {[-]} \\ \mathrm{P} & \text { pressure } & {\left[\mathrm{N} \mathrm{m}^{-2}\right]} \\ \mathrm{R} & \text { universal gasconstant } & {[-]} \\ \mathrm{R}_{\mathrm{uu}, \mathrm{vv}, \mathrm{ww}} & \text { spanwise autocorrelations of streamwise, } & \\ & \text { wall-normal and spanwise velocity component } & {[-]} \\ \mathrm{Re} & \text { Reynolds number } & {[-]} \\ \mathrm{St} & \text { Stokes number } & {[-]} \\ \mathrm{T} & \text { temperature } & {\left[\mathrm{K}^{-1}\right.} \\ \mathrm{U} & \text { velocity } & {\left[\mathrm{m} \mathrm{s}^{-1}\right]} \\ \mathrm{X}^{*} & \text { distance from mean position of the reflected shock normalized by } \\ & \text { interaction length } & {[-]} \\ \mathrm{W}_{\mathrm{s}} & \text { correlation window size } & {[\mathrm{pix} .]}\end{array}$

\section{Operators}

$\begin{array}{ll}<\bullet & \text { root mean square } \\ \bullet & \text { mean } \\ \bullet & \text { fluctuating value }\end{array}$

\section{Subscripts}

$\begin{array}{ll}\mathrm{e} & \text { static } \\ \mathrm{i} & \text { incompressible } \\ \mathrm{p} & \text { particle } \\ \mathrm{t} & \text { total } \\ \mathrm{W} & \text { wall } \\ \infty & \text { freestream }\end{array}$

\section{Abbreviations}

$\begin{array}{ll}\text { CCD } & \text { charged-coupled device } \\ \text { FOV } & \text { field-of-view } \\ \text { OF } & \text { overlap factor } \\ \text { PIV } & \text { particle image velocimetry } \\ \text { RMS } & \text { root mean square } \\ \text { SWTBLI } & \text { shock wave/turbulent boundary layer interaction } \\ \text { SBVG } & \text { sub-boundary vortex generator } \\ \text { VG } & \text { vortex generator }\end{array}$




\section{Chapter 1: Introduction}

\subsection{Motivation and relevance}

Shock wave/turbulent boundary layer interactions (SWTBLIs) are a class of fluid dynamic phenomena that are prevalent in many applications, e.g. helicopter blades, supersonic inlets, missile and aircraft after bodies, etc (Dolling 2001). The interactions are an important source of drag and can cause turbulent and unsteady separation of the boundary layer, leading to increased heat fluxes and fluctuating pressure loads, which can be severe enough to cause structural damage and premature fatigue of aero-structures (Dolling 2001; Smits and Dussauge 2006).

The dynamics of the unsteadiness exhibit a wide range of spatial and temporal scales including unsteady motions of the reflected shock foot with a frequency that is about two orders of magnitude lower than the incoming boundary frequency $\mathrm{U}_{\infty} / \delta$ where $\mathrm{U}_{\infty}$ is the freestream velocity and $\delta$ is the boundary layer thickness (e.g. Dussauge et al. 2006; Dupont et al. 2006; Souverein et al. 2008). To date much attention has focussed on documenting the characteristics of this low-frequency unsteadiness of the reflected shock and identifying its causes (Délery and Marvin 1986; Dolling 2001; Smits and Dussauge 2006). Despite all research there are currently no known models that can accurately predict every property of the phenomenon and even some fundamental aspects are not fully understood.

There is a growing number of studies that provide evidence for a model in which the lowfrequency component of the unsteadiness is driven by large-scale variations in the streamwise velocity of the incoming boundary layer (e.g. Beresh et al. 2002; Ganapathisubramani et al. 2007; Humble et al 2008). In particular, studies that made use of velocity data in wall parallel planes have led to renewed insight in the role of variations in the incoming boundary layer on the interaction's phenomenology (Ganapathisubramani et al. 2007; Humble et al 2008). These studies were assisted by the advent of advances in particle image velocimetry (PIV), which made it possible to probe certain aspects of the flow that could not be quantitatively explored before.

In order to minimize the detriments of SWTBLIs, a variety of flow control stratagies have been developed (Délery 1985). One of such strategy is to change the characteristics of the incoming boundary layer in a suitable way in order to diminish the detrimental effects of the shock induced separation (Délery 1985). A way to do this is by placing vortex generators (VGs) upstream of the SWTBLI. Conventional VGs with a height in the order of the boundary layer thickness have been used for some time to provide control in supersonic applications (Pearcey 1961).

Recently attention has focused on sub-boundary layer VGs (SBVGs), which have a height of about $10-40 \%$ of the undisturbed boundary-layer thickness and thereby cause less drag than conventional VGs (Lin 2002). One particular type of SBVGs is the micro-ramp. Micro-ramps are very robust and easy to apply compared to other SBVGs. Furthermore, they have been shown to reduce the length of the separated region, causing a more abrupt pressure rise (e.g. McCormick 1993; Barter and Dolling 1995; Pitt Ford and Babinsky 2007) and to stabilize the interaction (Anderson et al. 2006). 


\subsection{Thesis aim and objectives}

The aim of this thesis is to investigate the effects of micro-ramps on the flow organization of a SWTBLI. This aim will be met through the following objectives:

- to apply of oil-flow visualization and stereo-PIV in planes parallel to the surface to the SWTBLI problem in cases with and without micro-ramps.

- to make comparisons between the mean flowfields, instantaneous flow organization and turbulence properties,

- to perform a statistical analysis to further investigate the effects micro-ramps on the flow structure.

- and finally, to develop a conceptual model of the effects of the micro-ramps on the flow structure.

\subsection{Outline of the thesis}

In the next section an introduction to SWTBLIs and their control by micro-ramps will be given. Subsequently, in the chapter 3 the experimental techniques and set-ups used are described. Thereafter in chapter 4 the results of the experiments will be presented and discussed. The major observations made in chapter 4 will be consolidated to formulate a conceptual model in chapter 5. Finally, a summary of the conclusions and recommendations is given in chapter 6 . 


\section{Chapter 2: Background of SWTBLIs and their Control by Micro-Ramps}

\subsection{Types of SWTBLI}

In order to provide the reader with some examples of SWTBLIs in practical flows of interest, figure 1 and figure 2 show cross-sectional schematics of a transonic airfoil at cruise (incident normal shock interaction) and an intake to supersonic jet engine (compression surface interaction, incident oblique and incident normal shock interaction). SWTBLIs in practical flows are often three-dimensional on the mean (Smits and Dussauge 2006): the shock wave is swept with respect to the boundary layer and/or the boundary layer itself might be threedimensional.

SWTBLIs are usually classified according to how the shock is generated (Smits and Dussauge 2006). The shock may impinge on the boundary layer from an external source (an incident shock interaction) or the shock may be generated by the same surface on which the boundary layer develops (a compression surface interaction). This paper will focus on interactions that belong to the first type of interactions: oblique shock reflections on a flat plate.

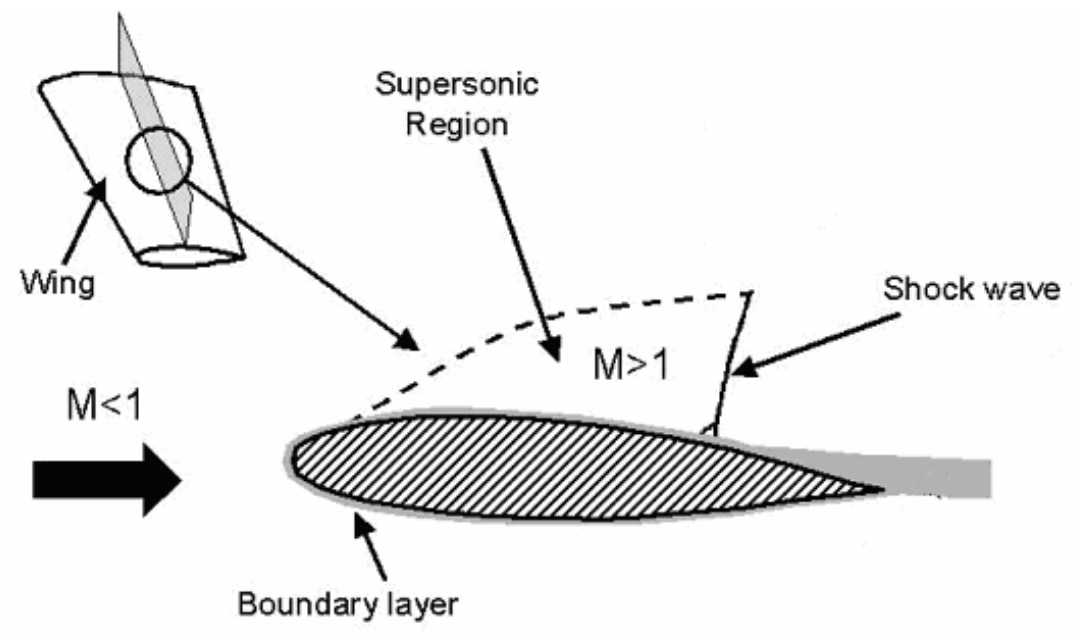

Figure 1: SWTBLI on a transonic airfoil at cruise (Holden and Babinsky 2007)

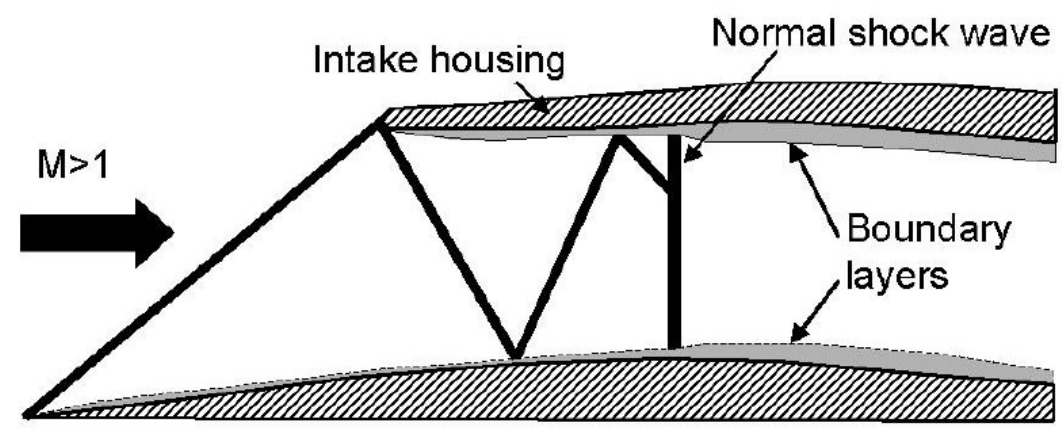

Figure 2: SWTBLI in a supersonic jet intake (Holden and Babinsky 2007) 


\subsection{Present interaction phenomenology}

According to the inviscid flow model, an oblique shock reflection constitutes of an incident shock that usually reflects of the wall as another oblique shock, such that the flow becomes parallel to the wall. In reality however, boundary layers are present which prohibit the shock from reaching the surface. For weak shocks the flow closely resembles the inviscid flow solution: the boundary layer remains attached and the interaction is well embedded within the boundary layer. Stronger shocks however can cause the boundary layer to separate leading to great deviations from inviscid model. If the incident shock strength further increases, a Mach stem will form and a quasi-normal shock will appear in the flow field.

Studies of the influence of shock strength, Mach number, Reynolds number and the state of the incoming boundary layer on the mean flow organization have been conducted (reviews can be found in Green 1970, Adamson and Messiter (1980) and Délery and Marvin (1986)). In this section, first the unseparated case will be discussed. Thereafter follows a discussion on the separated case.

\subsubsection{Unseparated case}

An interaction without separation of the boundary layer is depicted in figure 3 . The shock impinges on the boundary layer and then bends towards the wall because of the variation in the Mach number across the layer. Because the pressure rise caused by the impinging shock is transmitted upstream through the subsonic part of the boundary layer, the presence of the shock in the boundary layer is felt upstream of the point where the shock would impinge in the inviscid case. The rise in pressure results in thickening of the subsonic part of the boundary layer, which induces compression waves in the adjacent supersonic layer. These waves first weaken the incident shock wave and then coalesce to form the reflected shock. After thickening the subsonic part contracts, due to acceleration of the inner region of the boundary layer by viscous forces which progressively become predominant again (Délery and Marvin 1986). The refraction of the incoming shock as it propagates through the supersonic part of the boundary layer induces secondary waves. These waves are reflected by the sonic line into expansion waves.

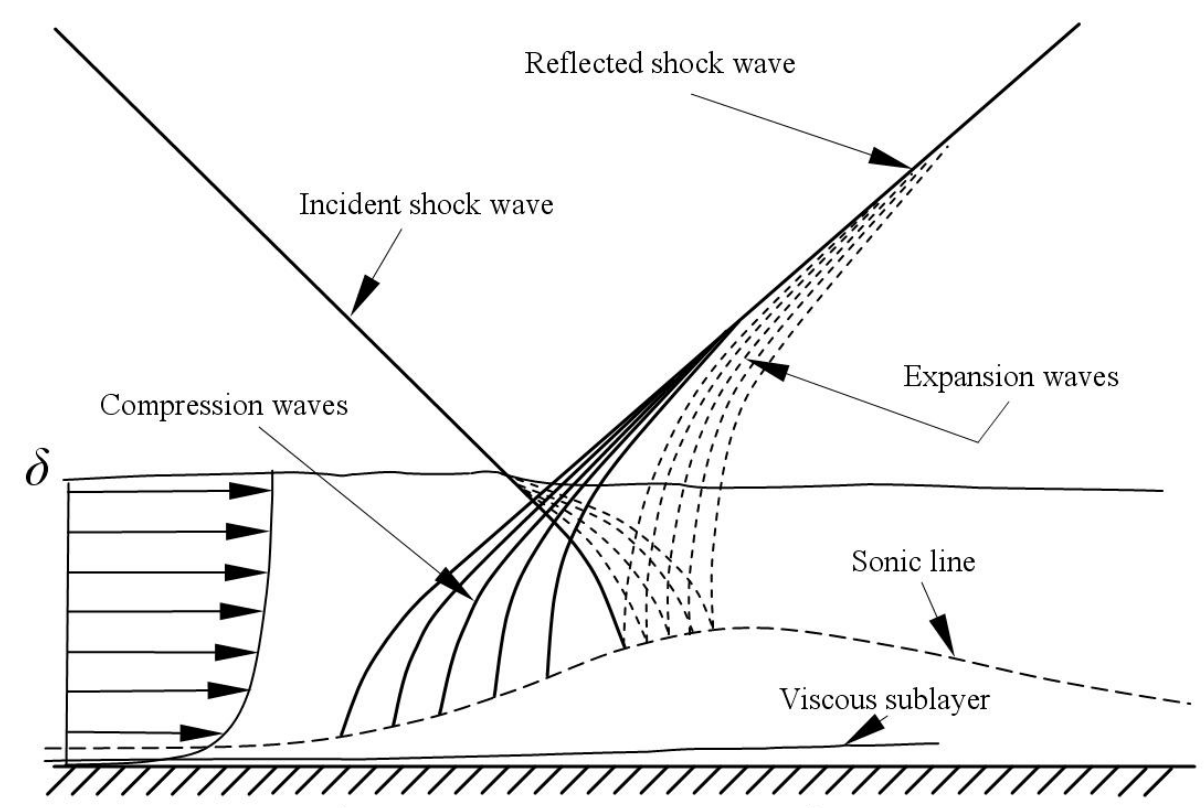

Figure 3: schematic of a shock reflection without separation (Humble 2008) 
For the unseparated case, the interaction length is the streamwise distance between the most upstream point where the pressure rise caused by the shock is felt and the location where the incident shock would impinge on the wall in absence of a boundary layer (Délery 1985; Délery and Marvin 1986). This distance is also called the steady upstream propagation length.

\subsubsection{Separated case}

An interaction with separation of the boundary layer is depicted in figure 4 . The location where the streamline leaves the surface is denoted as point S. Note that this point is located upstream of the location where the incident shock would meet the wall in absence of the boundary layer. Like in the unseparated case, the upstream propagation of the pressure rise induces compression waves in the supersonic part of the field, which coalesce to form the reflected shock. The reflected shock meets the incident shock which leads to a shock-shock interaction. The entropy rise through the shocks above this interaction is generally not the same as the entropy rise through the shocks below the interaction. This difference in entropy results in a slip-line (not depicted here).

A shock resulting from the shock-shock interaction impinges into the boundary layer in which it curves due to the change in local Mach number. The shock penetrates the layer up to the sonic line from which it is reflected as a system of expansion waves. The expansion fan results in a deflection of the flow towards the surface on which reattachment takes place at a point $\mathrm{R}$. The deflection and compression associated with reattachment is more spread out than at separation (Délery and Marvin 1986).

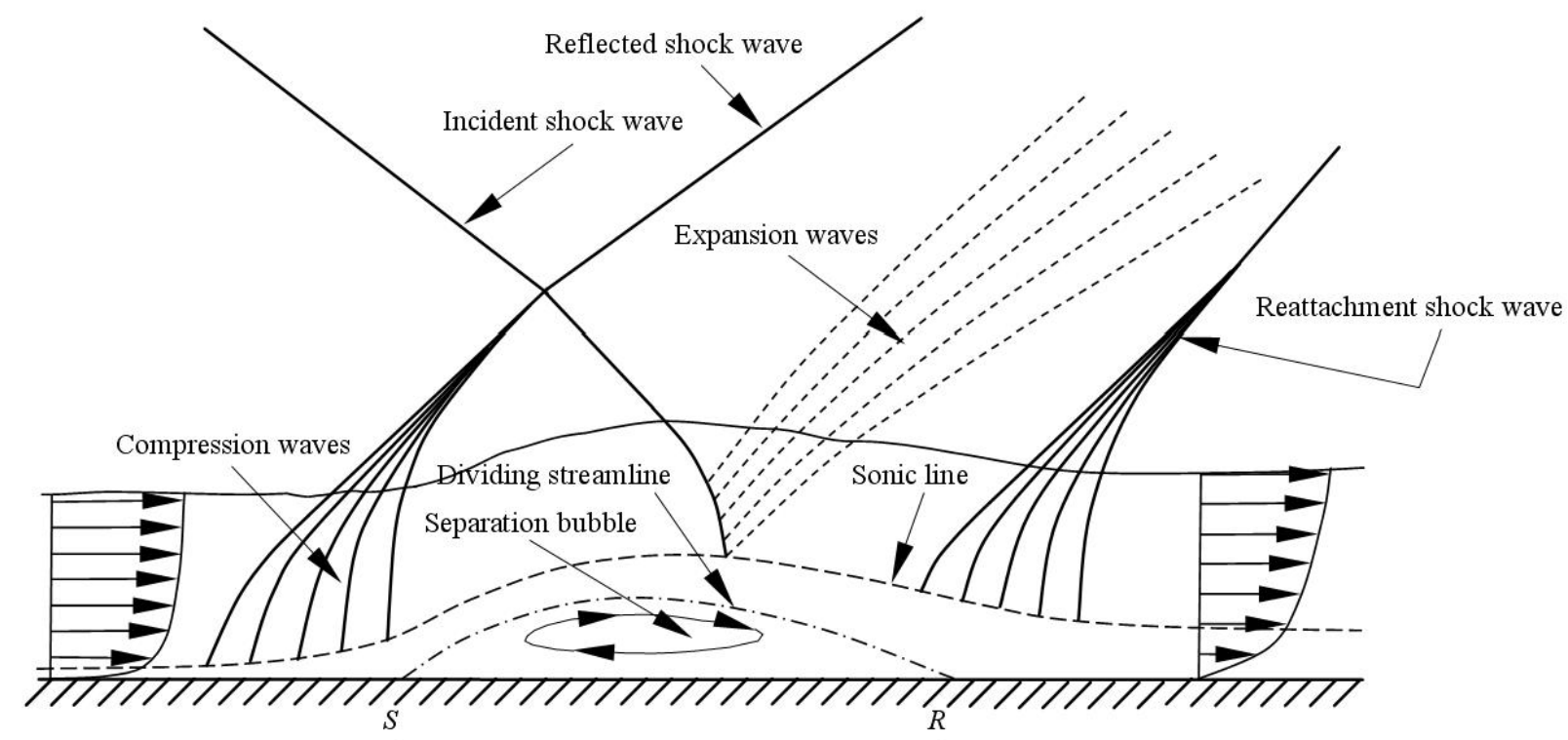

Figure 4: schematic of a shock reflection with separation (Humble 2008).

For the separated case the length of an interaction is defined as the streamwise distance between the mean position of the reflected shock and the point where the incident shock would impinge the wall in absence of a boundary layer (Dussauge et al. 2006; Dupont et al. 2006). A distinction is made between mean flow separation and instantaneous separation. Mean flow separation occurs on the point where the flow is separated $50 \%$ of the time (Simpson 1981) or in other words, where the intermittency is $50 \%$, where the term intermittency $(\gamma)$ is used to denote the fraction of the time that the separation shock is located upstream of a given point. It should be noted that there are interactions in which instantaneous 
separation can be observed, while no mean separation occurs (Humble et al 2007). In addition there is the concept of incipient separation, which can be seen as the onset of mean flow separation: the mean skin friction becomes zero at some point, but the flow does not actually separate.

In a steady two-dimensional model of the flow, separation can be defined relatively clearly: the instantaneous separation point is located at the point where the skin friction is zero and the stream surface leaves the wall. In three-dimensional flows the concept of separation is less clear. Separation occurs only over a finite spanwise length and three-dimensional separated region may only have two points where the skin friction is zero (Smits and Dussauge, 2006).

\subsection{Unsteadiness}

While in the above steady phenomenology description is relatively clear, in reality the interaction is unsteadyand its physical description is much more complicated. In interactions without boundary layer separation, unsteadiness only plays a small role (Smits and Dussauge 2006). Experimental observations of compression ramp interactions suggest that the reflected shock is rather steady apart from some minor oscillations due to incoming small-scale turbulence (Smits and Dussauge 2006). The length of the shocks motion remains smaller than about $0.1 \delta$ (Dolling 2001). In case separation occurs however, studies have shown that the interaction is accompanied by unsteady motions that exhibit a wide range of spatial and temporal scales (Dolling 2001).

The unsteadiness properties of the incident shock wave have less well been documented than those of the compression ramp interaction. Review papers that deal with the unsteadiness of the compression ramp interaction have been written by Dolling (2001) and Smits and Dussauge (2006). A comprehensive study of an oblique shock reflection for different shock intensities at Mach 2.3 has been performed by Dupont et al. (2006), who found similarity in the characteristic space and time scales of the two interaction types.

A result of their study is shown in figure 5 where the wall pressure power spectral density along an interaction is plotted. The shock was sufficiently strong to separate the boundary layer. The dimensionless coordinate in this figure $\mathrm{X}^{*}$ is the distance from the mean position of the reflected shock normalized the interaction length. The frequency ranges of interest can be arbitrarily distributed in into four distinct zones (Dupont et al. 2006):

The most upstream zone $\left(\mathrm{X}^{*}<-0.2\right.$ in figure 5) contains energetic frequencies larger than 10 $\mathrm{kHz}$ and can be associated with the incoming turbulent boundary layer. Dupont et al. (2006) found no significant energy in the range $100 \mathrm{~Hz}-1 \mathrm{kHz}$.

The second region $\left(-0.15<\mathrm{X}^{*}<0.15\right.$ in figure 5$)$ is associated with the intermittent region, which is defined as the region between the two extreme positions of the reflected shock. The length of the intermittent region is of the order of the undisturbed boundary layer thickness (Dolling 2001; Smits and Dussauge, 2006; Dupont et al. 2006) and becomes smaller for larger distances from the wall (Dupont et al. 2006; Humble et al. 2008). The motion of the reflected shock foot has a mean frequency that is about two orders of magnitude below the incoming boundary frequency $\left(\mathrm{U}_{\infty} / \delta\right)$ (Dolling 2001; Smits and Dussauge 2006; Dupont et al. 2006). The amplitude of the shock foot's movement increases almost linearly with shock intensity, while its frequency decreases for stronger shocks (Dupont et al. 2006). Dupont et al. (2006) 
found that the mean velocity of the shock foot is two orders of a magnitude lower than the freestream velocity and concluded that this low value suggests that it is unlikely that particular events could be produced by the shock motion.

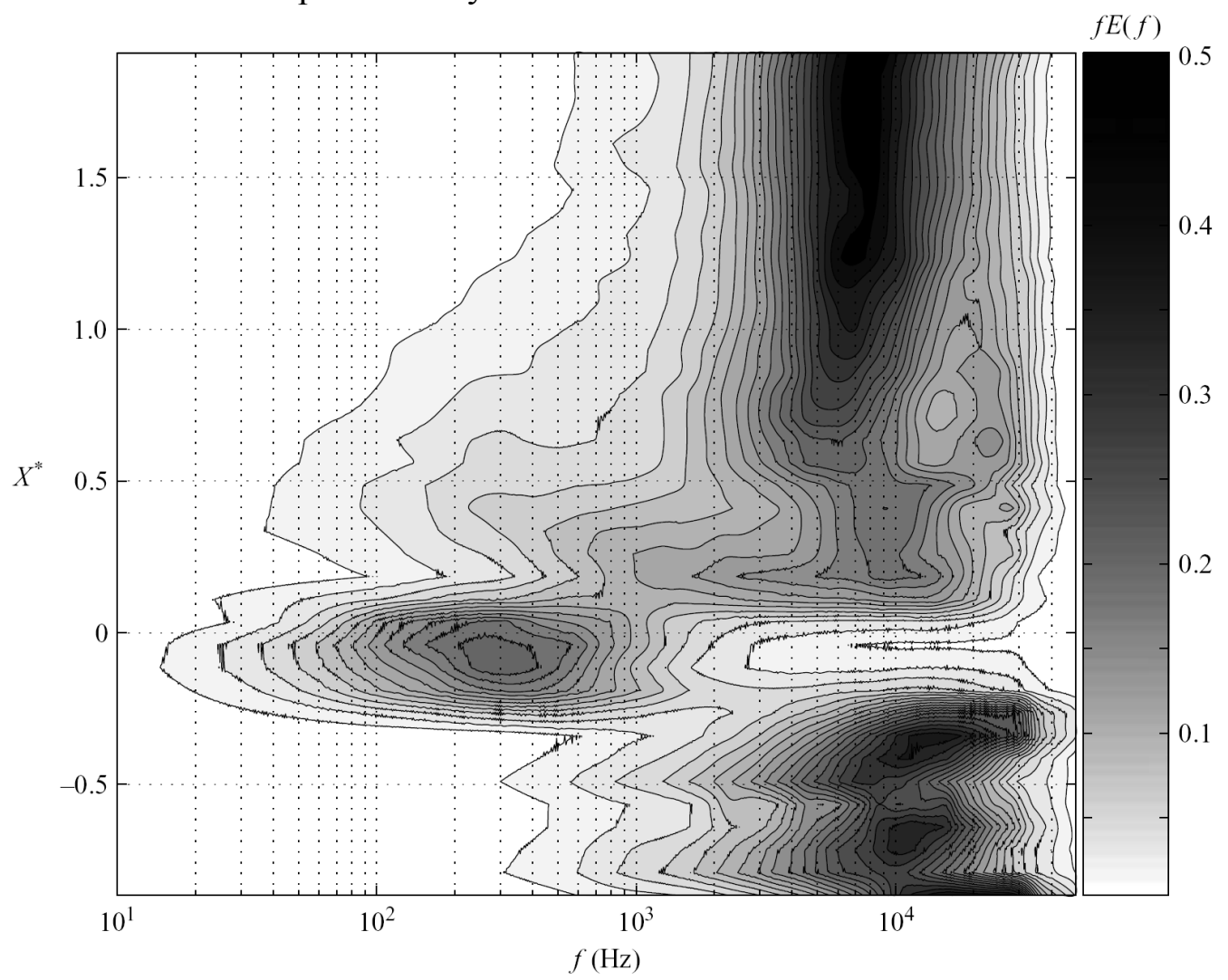

Figure 5: wall pressure power spectral density along the interaction; shock generator angle $\theta=8^{\circ}$ Due to limitations of the used wall pressure transducers, the maximum frequency is strongly underestimated (Dupont et al. 2006).

The third zone $\left(0.2<\mathrm{X}^{*}<1\right.$ in figure 5$)$ is associated with the separated region and characterized by intermediate scales developed in the frequency range $1 \mathrm{kHz}-10 \mathrm{kHz}$. Lower frequencies are still significant and represent about $20 \%$ of the signals total energy in this region (Dupont et al. 2006). The pressure fluctuations in this zone were found to have features in common with a mixing layer. Selig et al. (1989) (as reported by Smits and Dussauge (2006)) found that the high turbulence levels near the edge of the separation bubble match the high levels of turbulence found in mixing layers. Dupont et al. (2008) performed PIV measurements of incident shock interactions under similar conditions as Dupont et al. (2006) and reported that the velocity profiles in separated flow are similar to those encountered in mixing layer. Further evidence of the development of a mixing layer zone was found after processing of the velocity profiles by means of a statistical vortex detector.

The fourth and final zone in figure $5\left(X^{*}>1\right)$, was described by Dupont et al. (2006) as a relaxation zone where both medium and high frequencies are present. The medium frequencies that where developed inside the third zone are dominant up to sections far downstream of the reattachment point (Dupont et al. 2006). 


\subsection{Source(s) of low-frequency unsteadiness}

The low-frequency motion of the shock motion has received much attention in literature. Despite all research the driving mechanisms involved in the low-frequency dynamics of the interaction are still not fully understood. Some researchers related the shock motion to the downstream conditions. Erengil and Dolling (1991) performed wall pressure measurements upstream of a compression ramp in a Mach 5 flow and proposed a model in which fluctuations in the incoming boundary layer are responsible for the high-frequency component of the shock motion, whereas the low-frequency component is related to some large-scale instability of the separated bubble. Supportive of this last reported relation are the results of Thomas et al. (1994), who found a close-to-one coherence between the shock motion and the dynamics of the separated region in a compression ramp interaction at Mach 1.5 based on wall pressure measurements. However, In contrast to results of Erengil and Dolling (1991), they found no discernable statistical relationship between burst-events in the incoming boundary layer and the shock motion

Dussauge et al. (2006) reviewed and analysed an incident shock interaction by evaluating results of various experiments. Based on a scaling of the characteristic frequency of the shock motion, they stated that eddies in the separated region may be the source of excitation of the shock. Furthermore, they put in evidence that three-dimensional structures encountered in the separated bubble have characteristic time scales in the same order as the low-frequency component of the shock motion and suggested that these structures may be the origin of the low-frequency unsteadiness.

As mentioned in the previous section, Dupont et al. (2008) found the development of a mixing layer in the separated region that is accompanied by large amplitude vertical motions in the order of one boundary layer thickness. This was associated with flapping of the boundary layer, which appeared to have significant correlation with the reflected shock movements, suggesting that the mixing layer dynamics is related to the low-frequency shock motions.

Recently, a relation between the shock motion and the dynamics of the downstream flow has also been found by numerical studies of the interaction (see Edwards 2008 for a recent overview). After performing direct numerical simulations (DNS) of an incident shock interaction, Pirozzoli and Grasso (2006) proposed an acoustic feedback mechanism in the interaction region. A study of DNS data of a compression ramp interaction by $\mathrm{Wu}$ and Martin (2008) showed correlations among the spanwise mean separation point, the reattachment point and the shock location that indicate that the low-frequency shock unsteadiness is influenced by the downstream flow. Moreover, by means of conditional statistics they found no significantly change in the properties of the incoming boundary layer for different shock positions.

Other researchers sought for a dependency of the low-frequency shock motion on velocity fluctuations in the incoming boundary layer. Plotkin (1975) proposed a mathematical model for this dependency. He assumed that a stable location exists for the shock. This is the position where the shock would be located if no disturbances were present in the incoming flow. As an organized structure convects into through the interaction, it changes the jump conditions across the shock, and causes it to move away from the equilibrium position. After the structure passes, the stability of the mean flow moves the shock back towards its equilibrium position through a linear restoring mechanism. 
Poggie and Smits (2001) evaluated Plotkin's model with measurements in a reattaching shear layer and showed that there where the shock motion is dominated by the low-frequency component, the model was found to give an excellent fit. Smits and Dussauge (2006) however point out that although the model predicts a respond that is consistent with measured data, it does not provide proof for the assumptions used by Plotkin (1975). Neither does the model provide a clear physical cause for the large-scale unsteady dynamics of the interaction.

McClure (1992) and Ünalmis and Dolling (1998) both combined the pressure signal of a Pitot-tube upstream of a compression interaction with wall pressure signals under the shock induced separation region. Their results suggested that the low-frequency shock motion is induce by variations in the incoming boundary layer thickness. Like Erengil and Dolling (1991), Beresh et al. (2002) investigated a compression ramp interaction at Mach 5. They combined wall pressure measurements under the intermittent region with PIV measurements of the incoming boundary layer and found no measurable difference in boundary layer thickness for different shock positions.

They did however find results suggesting that continuous variations in the upstream velocity fluctuations cause an unsteady shock foot motion according to the following mechanism (see figure 6): when positive velocity fluctuations occur in the incoming boundary layer, the velocity profile is momentarily fuller, which imparts an increased resistance to separation that yields a downstream motion of the separation shock. Conversely, a negative velocity fluctuation results in an upstream shock motion as the interaction responds to the less-full shape of the velocity profile. The results of Beresh et al. (2002) are in general agreement with numerical results of Hunt and Nixon (1995), who showed an approximately a one-to-one relationship between the fluctuations in the incoming boundary layer and the shock motion.
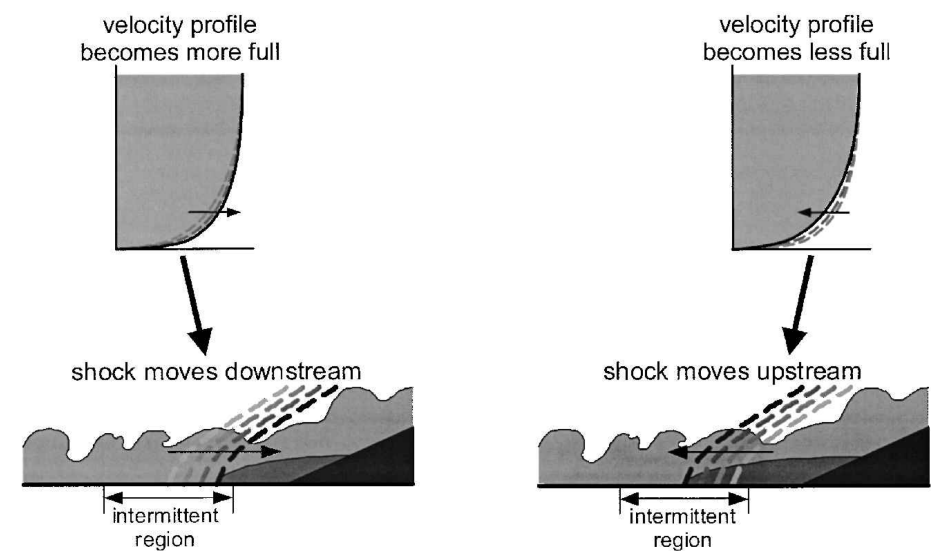

Figure 6: relationship between the incoming turbulent boundary layer and the separation shock foot unsteadiness (Beresh et al. 2002).

In order for structures in the incoming boundary layer to be the source of the low-frequency dynamics of the interaction, they need to be more than an order of a magnitude larger than the boundary layer thickness. In search for such large structures Ganapathisubramani et al. (2006) investigated a supersonic boundary layer in a Mach 2 flow by means of PIV in steamwisespanwise planes. They found elongated regions of high- and low-speed fluid with streamwise lengths greater than $8 \delta$. Using planar laser scattering (PLS) and Taylor's hypothesis Ganapathisubramani et al. (2007) even found regions up to $40 \delta$ long, meandering about 1-2 $\delta$ in spanwise direction. Supportive to these findings are the results of Elsinga (2008), who performed tomographic PIV measurements of a supersonic boundary layer at the same 
freestream Mach number and showed the existence of elongated three-dimensional low-speed regions. Further evidence for the existence of large structures in supersonic boundary layers comes from Ringuette et al. (2008) who performed direct numerical simulations of turbulent boundary layers at Mach 3 and found low-speed structures with streamwise lengths in the order of a $100 \delta$. The presence of these elongated structures is consistent with results of studies on incompressible boundary layers (Kim and Adrian 1999; Hutchins and Marusic 2007; Tomkins and Adrian 2003 and Ganapathisubramani et al. 2003).

In a continuation of the work of Ganapathisubramani et al. (2006), Ganapathisubramani et al. (2007) investigated the effect of these elongated regions on a compression ramp interaction and observed the following relation between the instantaneous position of the separation line and the size of the separated region: if the incoming boundary layer is relatively fast, the separated region is pushed back downstream leading to a smaller separation region. Conversely, if the incoming boundary layer is slow, the separated region is pulled upstream, leading to a larger separated region. Note that this behaviour is in complete agreement with the earlier discussed model proposed by Beresh et al. (2002). The same behaviour was also found in an incident shock interaction at Mach 2 by Humble et al. (2008), who investigated its instantaneous three-dimensional flow organization by means of tomographic PIV. Furthermore, they reported that the rippling of the reflected shock foot seems to follow the spanwise periodicity of the coherent motions in the incoming boundary layer.

\subsection{Interaction control techniques}

As mentioned earlier, SWTBLIs can be an important source of drag and of other unfavourable effects such as high heat fluxes and fluctuating pressure loads. In order to minimize these detriments, different flow control techniques have been developed. Typically, the aim of SWTBLI control is either to reduce the losses due to drag, to suppress the separation in order to obtain a steadier flow field or a combination of both.

Reduction of drag losses is generally achieved by smearing of the shock. This approach requires some subtleness, because devices that accomplish shock smearing tend to increase the friction drag (Délery 1985). The intended result of shock smearing is therefore to attain a new balance between these two, such that the total drag becomes less than in the uncontrolled configuration.

Suppression of separation can be achieved either by locally changing the organization of interaction or by changing the properties of the incoming boundary layer such that it becomes less susceptible to separation. The latter is typically done by increasing the shape factor of the boundary layer (Délery 1985). The shape factor is a measure for the fullness of the velocity profile or in other words for the amount of kinetic energy in the boundary layer. A boundary layer with fuller velocity profile has a lower value of the shape factor. Increasing the shape factor will often result in an intensification of the shock, hence an increase of the wave drag (Délery 1985).

Below follows a summation of both active and passive control techniques that can commonly be found in literature. Also combinations of these techniques are possible. The most efficient method in terms of weight and costs depends on the application. Review papers that deal with control of SWTBLIs have been written by Pearcey (1961), Délery (1985), Raghunathan (1988), Délery (2000) and Délery and Bur (2000). 
Control techniques aimed at reduction of drag losses:

\section{Passive cavity}

The principle of the passive cavity consists of establishing a natural circulation between the downstream high-pressure face of a shock and its upstream low-pressure face. This circulation spreads the shock system while reducing boundary layer thickness. It is achieved by placing a cavity underneath the shock foot region. The face in contact with the outer flow is typically covered by a perforated plate (Bur et al. 2000; McCormick 1993), although the use of mesoflaps has also been investigated (Gefroh et al. 2002).

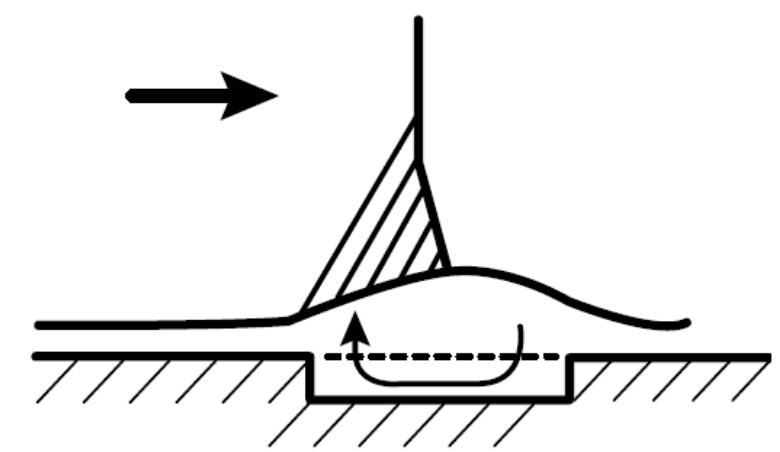

Figure 7: concept of SWTBLI control by a passive cavity (McCormick 1993)

\section{Local deformation of the surface}

Local deformation of the surface which materializes the viscous separated fluid of the original interaction appears to be very effective to spread the compression and does not have a large effect on the boundary layer. Thus friction losses are limited, while there is a gain due to wave drag reduction (Délery and Bur, 2000; Holden and Babinsky 2005).

\section{Three-dimensional surface modification}

Smearing of the shock has also been achieved by a variety of three-dimensional surface deformations at the shock location: bumps, slots, and grooves. These control devices act according to the same principle as the two-dimensional cavity and/or deformation. They were reported to provide beneficial shock smearing over a wide area, while confining viscous losses to narrow regions (Smith et al. 2002; Holden and Babinsky 2003; Holden and Babinsky 2005).

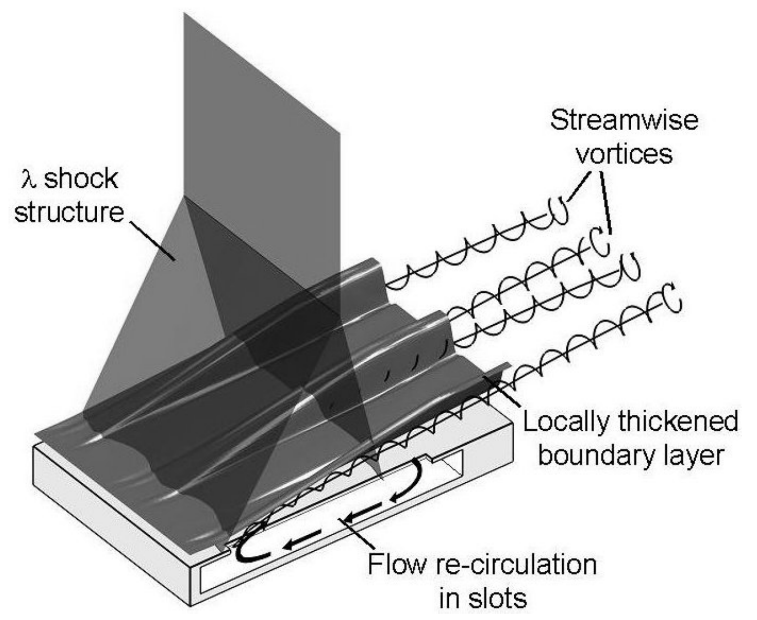

Figure 8: concept of SWTBLI control by streamwise slots (Smith et al. 2002) 
Control techniques aimed at suppression or reduction of separation by changing the properties of the incoming boundary layer:

\section{Mass injection by wall jets}

These jets can be placed tangential, adding momentum to the flow as was done by Peake 1966) or placed pitched/skewed. Positioned this way the jets are also called vortex generator jets (VGJs) as they work according to the same principle as vortex generators, which is described below (Pearcey 1961; Bueno 2006).

\section{Suction/bleeding}

Strong suction at a well chosen location upstream of the interaction can eliminate the low speed part of the boundary layer. Also distributed suction applied over a certain part of the boundary layer upstream of the interaction leads to a fuller velocity profile. Distributed suction or bleeding is often used in supersonic inlets, even though for this particular application it gives cause to a drag penalty by reducing the engine mass flow, (Délery 2000).

\section{Wall temperature control}

A decrease in wall temperature increases the local Mach number and as a result reduces the extent of the subsonic layer by decreasing the speed of sound. The upstream propagation of the shock influence will be diminished by this effect. In addition, a change in walltemperature changes the density of the gas in the boundary layer resulting in a change of boundary layer momentum: favourable in the case of cooling, unfavourable in the case of heating. (Délery and Bur, 2000)

Vortex generators (VGs)

VGs have been used for more than 50 years to provide control in supersonic applications (Pearcey 1961). VGs come in many shapes and sizes, but they all aim to create vortex structures that operate a forced mixing of the boundary layer in order to energise the near wall flow. This reduces the shape factor of the boundary layer making it less susceptible to separation. Compared to other mentioned SWBTLI control techniques that change the properties of the incoming boundary layer, VGs are very cheap and usually easy to apply.

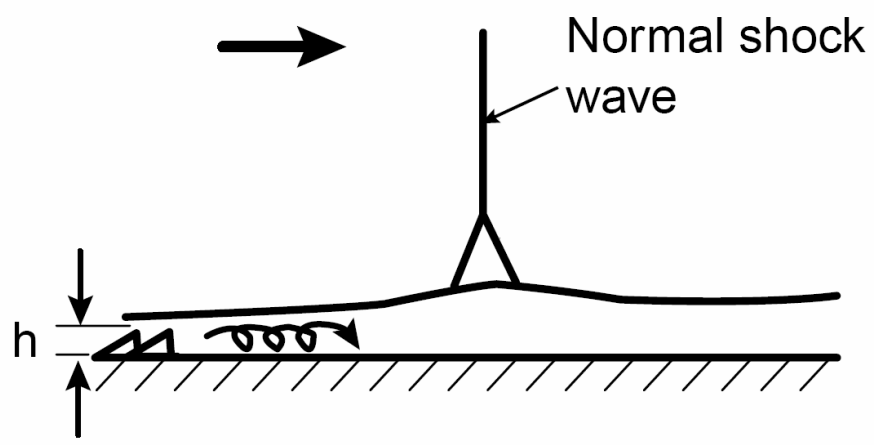

Figure 9: concept of SWTBLI control by low-profile vortex generators (McCormick 1993)

\section{Modification of wall contour}

Any modification of the wall contour leading to an acceleration of the flow, will stabilize the boundary layer by making its velocity profile fuller (Dolling 2001). 
Control techniques that aim to suppress or reduce separation by locally changing the organization of the interaction:

\section{Blowing/suction}

Blowing or suction through one or several slots near the shock location can increase the velocity on the separation streamline. Hereby, the ability of the flow to withstand an adverse pressure gradient without separating is augmented (Bur et al. 2000; Délery 2000).
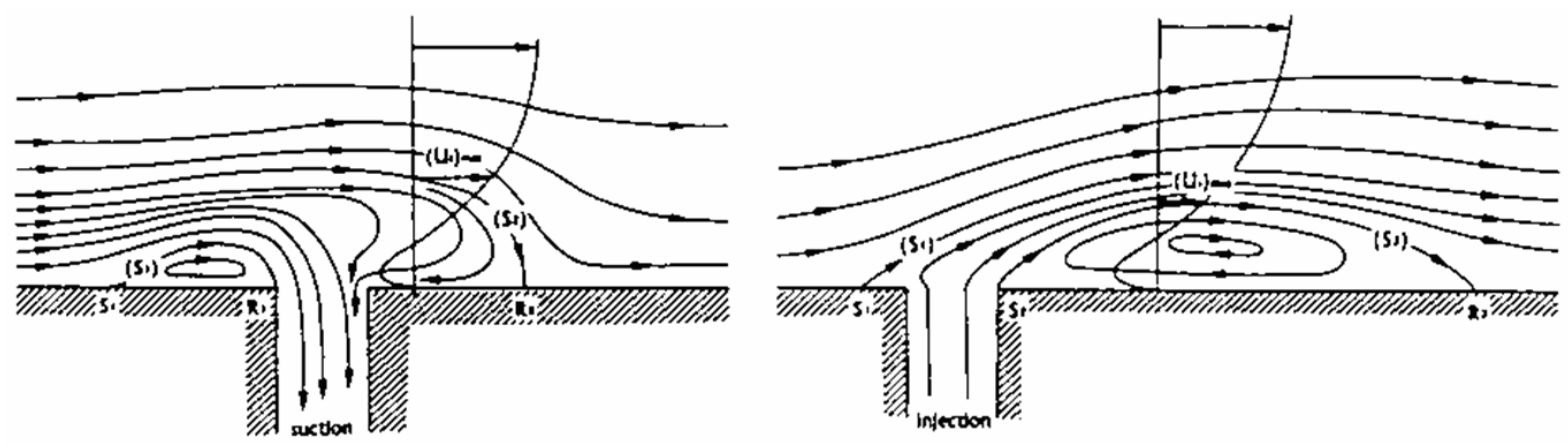

Figure 10: concept of SWTBLI control by blowing/suction near the shock location (Délery 2000)

\subsection{Developments towards micro-ramp control}

Since VGs were first introduced in the late 1940's, they have been used on numerous aircraft. They have been shown to reduce the extent of boundary layer separation in subsonic flow, but also in SWTBLIs (Pearcey 1961; Lin 2002), which has led to an increase in lift, improved stability and control characteristics, improved buffet margins and reductions in drag (Freestone 1995, Lin 2002). Conventional VGs have a device height in the order of the boundary layer thickness and commonly consist of vanes fixed at right angles to the main surface (see figure 11). Reviews on the use and design of conventional VGs are given by Pearcey (1961) and Freestone (1995).

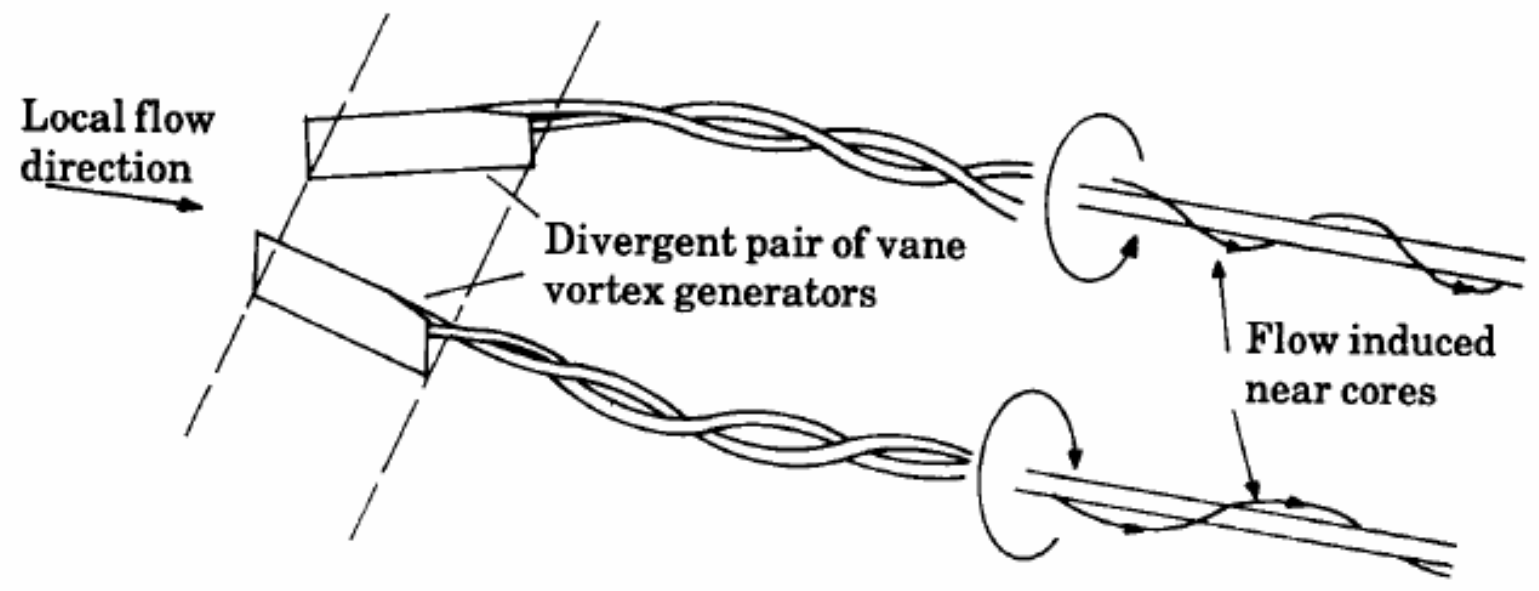

Figure 11: vane-type VGs placed in counter-rotating configuration (Freestone 1995)

Other shapes were proposed as well (see figure 12), many of which ceased to attract attention, such as the wedge, ramp, plough, scoop and dome, after it was found that they induce unnecessary drag by generating the vortices in a more inefficient way than the vane-type (Freestone 1995). 

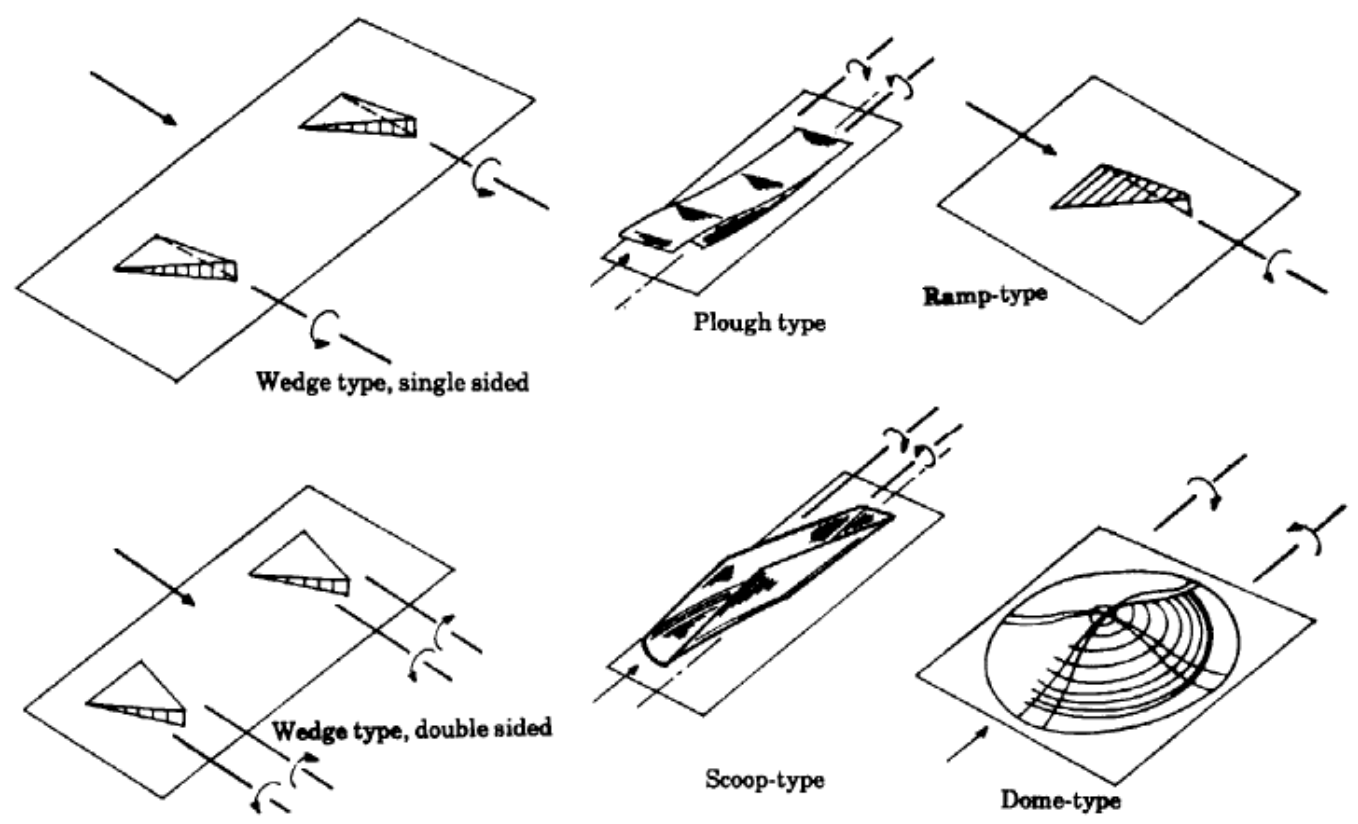

Figure 12: various early types of VGs (Freestone 1995)

An obvious disadvantage of the use of VGs is their inherent parasitic drag. For this reason VGs with lower profiles and therefore reduced drag were developed since the early 1970's (Lin 2002). These VGs had a device height of about $0.1 \delta-0.4 \delta$ and will from hereon be called sub-boundary layer VGs (SBVGs) following Ashill et al. (2001). Despite their low device height, SBVGs have been shown to significantly reduce shock induced separation (Ashill et al. 2001; McCormick 1993; Barter and Dolling 1995; Holden and Babinsky 2007; Pitt Ford and Babinsky 2007).

Non-conventional shapes of SBVGs were proposed by Wheeler (see figure 13) and Kuethe (not depicted here) (Freestone 1995). The former shape was used in an experiments by McCormick (1993) and Barter and Dolling (1995). McCormick (1993) placed Wheeler doublet SBVGs with a height of $0.36 \delta$ upstream of a normal shock interaction at $M=1.6$ and found that the devices reduced the interaction length and the reversed flow region by a factor 2-2.5. This is in agreement with results of an accompanying numerical study of Mounts and Barber (1992), which also showed a reduction in the extent of the reversed flow region.
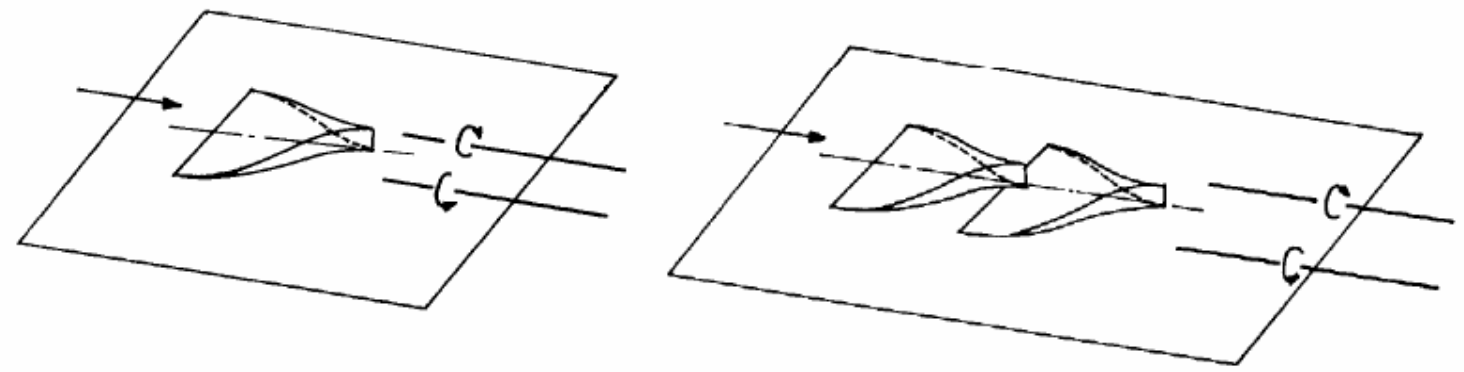

Figure 13: Wheeler singlet (left) and Wheeler doublet (right) (Freestone 1995)

Barter and Dolling (1995) found similar reductions as McCormick (1993) after placing Wheeler doublet SBVGs with a height of $0.33 \delta$ upstream of a compression ramp interaction at Mach 5. Another important result of Barter and Dolling (1995) was that the SBVGs decreased 
the amount of energy in the structural resonance frequency band decreased by about $50 \%$. To the author's knowledge this is the first and only experimental result that directly shows the effect of wedge-type SBVGs on the unsteadiness of the interaction.

Ashill et al. (2001) compared the effects of vane-type and wedge-type SBVGs of the same height on a transonic airfoil for different angles of attack and found that the vane-type SBVGs reduce the extent of separated flow relative to that of the wedge-type SBVGs.

Interest in the wedge-type SBVGs was renewed by Anderson et al (2006), who performed a parametric study on the control of an incident shock wave interaction using vane-type and wedge-type SBVGs at a freestream Mach number of 2.0 by means of RANS calculations. Anderson et al. (2006) used the terms micro-vanes and micro-ramps to denote vane-type and wedge-type SBVGs respectively; a convention that will also be used in the present investigation.

Anderson et al (2006) optimized the shape of the SBVGs for minimum shape factor downstream of the interaction and for maximum pressure difference over the interaction. After optimization it was found that both types of SBVGs could produce approximately the same reductions in the area averaged shape factor downstream of the interaction with comparable pressure recovery. However, Anderson et al (2006) implicitly showed that for a micro-ramp to be as effective as a micro-vane it has to have a larger height; a result that is in agreement with the former mentioned study of Ashill et al. (2001) and with results of Holden and Babinsky (2007). The latter placed micro-vanes and micro-ramps with heights of $0.23 \delta$ and $0.38 \delta$ respectively upstream of a normal shock interaction in a Mach 1.5 flow and found that the micro-vanes were more effective since they completely eliminated separation, while the micro-ramps only reduced the extent of the separated region. Using time accurate CFD solutions, Anderson et al. (2006) found that arrays of micro-ramps stabilize the SWTBLI which is in line with earlier described results of Barter and Dolling (1995).

Despite studies that indicate a larger effectiveness of the micro-vanes, interest in micro-ramps did not cease to exist, because micro-ramps are structurally highly robust and easier to apply than micro-vanes. These properties are particularly important in engine applications since manufacturers are unwilling to use fragile SBVGs that may break during service and cause damage to the engine (Pitt Ford and Babinsky 2007).

Having acknowledged these advantages of micro-ramp control, Pitt Ford and Babinsky (2007) experimentally investigated the effect micro-ramps, fabricated according to scalings of Anderson et al. (2006), on an impinging shock wave at Mach 2.5. It was found that the microramps lead to a smaller spanwise averaged incompressible shape factor, a smaller interaction length and shorter separated region. The conclusions of Pitt Ford and Babinsky (2007) are supported by results of a large eddy simulations (LES) performed by Lee et al. (2007). In this simulation, micro-ramps, again defined according to scalings of Anderson (2006), were located upstream of an impinging shock wave in a Mach 3 flow. Like in all former mentioned control studies it was found that the micro-ramps reduced the size of the separated flow region with respect to the undisturbed interaction. 


\subsection{Micro-ramp control phenomenology}

Experimental studies have put in evidence that like conventional VGs, micro-ramps generate pairs of counter-rotating vortices (Holden and Babinsky 2007; Pitt Ford and Babinsky 2007). The vortices are rotating such that in the region between them the induced flow is moving away the surface, sweeping away low-momentum fluid from the lower part of the boundary layer. At these spanwise locations the boundary layer was seen to be thicker (Barter and Dolling 1995; Holden and Babinsky 2007; Pitt Ford and Babinsky 2007) and have the largest momentum and displacement thickness (McCormick 1993; Pitt Ford and Babinsky 2007). Elsewhere higher-momentum fluid coming from regions farther away from the wall is induced downwards, replacing the swept away low-momentum fluid and locally thinning the boundary layer. Results of computational studies are in agreement with this flow organization (Mounts and Barber 1993; Anderson et al. 2006; Lee et al. 2007)

The flow organization described above is visualized in figure 14. The contours show the total pressure contours downstream of a micro-ramp array as computed by Anderson et al. (2006). The semi-transparent rectangular shapes represent the micro-ramps and the transparent arrows illustrate the streamwise vortices. The size of both the micro-ramps and the vortices is chosen arbitrarily.

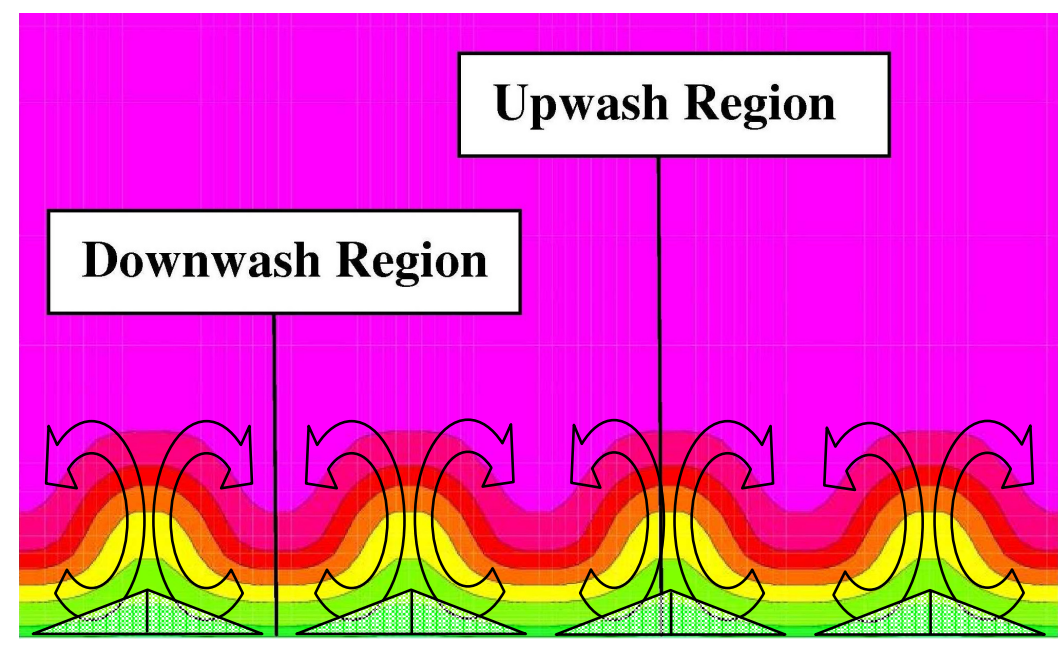

Figure 14: spanwise total pressure contours for a micro-ramp array (Anderson et al. 2006); drawn in semi-transparent rectangles depict the micro-ramps and the transparent arrows the streamwise vortices; size of micro-ramps and vortices is chosen arbitrarily.

The spanwise organization of the boundary layer has consequences for the control of the SWTBLI. Barter and Dolling (1995) found that downstream of their apices the SBVGs were least effective, leading to the longest interaction lengths at these spanwise locations. Pitt Ford and Babinsky (2007) found that the micro-ramps break up the two-dimensional separation zone of the undisturbed interaction into periodic three-dimensional zones that exist downstream of the vertices.

The degree and streamwise extent of micro-ramp control effectiveness is dependent on the size, shape and configuration of the micro-ramps. A theoretical investigation of this dependency was performed by Smith (1994) in order to develop a tool for their design. The developed model predicted experimental data well and envisaged that higher benefits in terms of vortex strength should come from increased spanwise packing, increased volume, and nonsmooth SBVG shapes (Smith 1994). The first two recommendations are in agreement with 
Anderson et al. (2006), who as mentioned earlier performed a parametric study in the field of micro-ramp design by means of numerical techniques. They found that the micro-ramps that minimized the shape factor downstream of the interaction were the largest simulated microramps with the highest simulated degree of spanwise packing. This result is in line with findings of Pitt Ford and Babinsky (2007), who experimentally investigated micro-ramps with heights varying from $25 \%$ to $75 \%$ of the boundary layer thickness, and showed that larger micro-ramps lead to a smaller spanwise averaged incompressible shape factor, a smaller interaction length and shorter separated region.

Another important design parameter is the distance between the micro-ramps and the SWTBLI. This distance should be such that the energization of the boundary layer is optimal at the SWTBLI (Pearcey 1961; Freestone et al 1995). Placed too close to the interaction, the vortices have not had sufficient time to energize the boundary layer (Mounts and Barber 1993). This was also implicitly shown by Anderson et al. (2006) who found that the most effective generator-to-shock spacing was the largest that was simulated.

Several researchers have reported a range of generator-to-shock spacings for which the benefits to the boundary layer are nearly constant McCormick 1993; Barter and Dolling 1995; Pitt Ford and Babinsky 2007). The existence of this range was already described and explained for conventional VGs by Pearcey (1961), who referred to it as the range of effectiveness.

Pearcey (1961) found that pairs of counter-rotating vortices such as found downstream of micro-ramps induce each other away from the surface. Furthermore, he stated that the mixing effect of the vortex pairs is primary related to the spacing of the vortices relative to their height above the wall and that therefore the streamwise extent of the effectiveness length is primarily related to the movement of the vortices in the cross-flow plane. Apart from the vortex migration away from the wall, the effectiveness of the micro-ramps is limited by growth of the viscous vortex cores and reduction of vortex strength by dissipation, which has its major causes in surface friction and eventual viscous interaction between two neighbouring vortex cores (Freestone 1995).

Throughout the description of vortex motion, size and strength we have relied on literature on conventional VGs. Generalization of this description of vortex motion to vortices generated by SBVGs can be done based on laser Doppler anemometry (LDA) measurements performed in subsonic flow (Ashill et al. 2002), oil-flow visualizations in supersonic flow (Pitt Ford and Babinsky 2007) and on numerical simulations of supersonic flow (Mounts and Barber 1992, Lee et al. 2007). 
EFFECTS OF MICRO-RAMPS ON A SHOCK WAVE BOUNDARY LAYER INTERACTION 


\section{Chapter 3: Experimental Techniques and Arrangement}

\subsection{Experimental facility}

Experiments were performed in the transonic-supersonic wind tunnel (TST-27) of the Delft University of Technology. This is a blowdown wind tunnel that can generate flows in the test section within the Mach number range 0.5 to 4.2. A schematic overview of the tunnel is depicted in figure 15 . The test section is $280 \mathrm{~mm}$ in width and $250 \mathrm{~mm}$ to $270 \mathrm{~mm}$ in height depending on the operated Mach number. The Mach number can be set by means of a continuous variable throat and flexible upper and lower walls. The facility automatically corrects small variations in the Mach number by fine adjustment of the choke. The maximum unit Reynolds number varies from $30 \times 10^{6} \mathrm{~m}^{-1}$ in the transonic range to $130 \times 10^{6} \mathrm{~m}^{-1}$ at Mach 4. Hot-wire anemometry measurements performed in the test section found a turbulence intensity of approximately $0.01 \mathrm{U}_{\infty}$ (Humble et al. 2008).

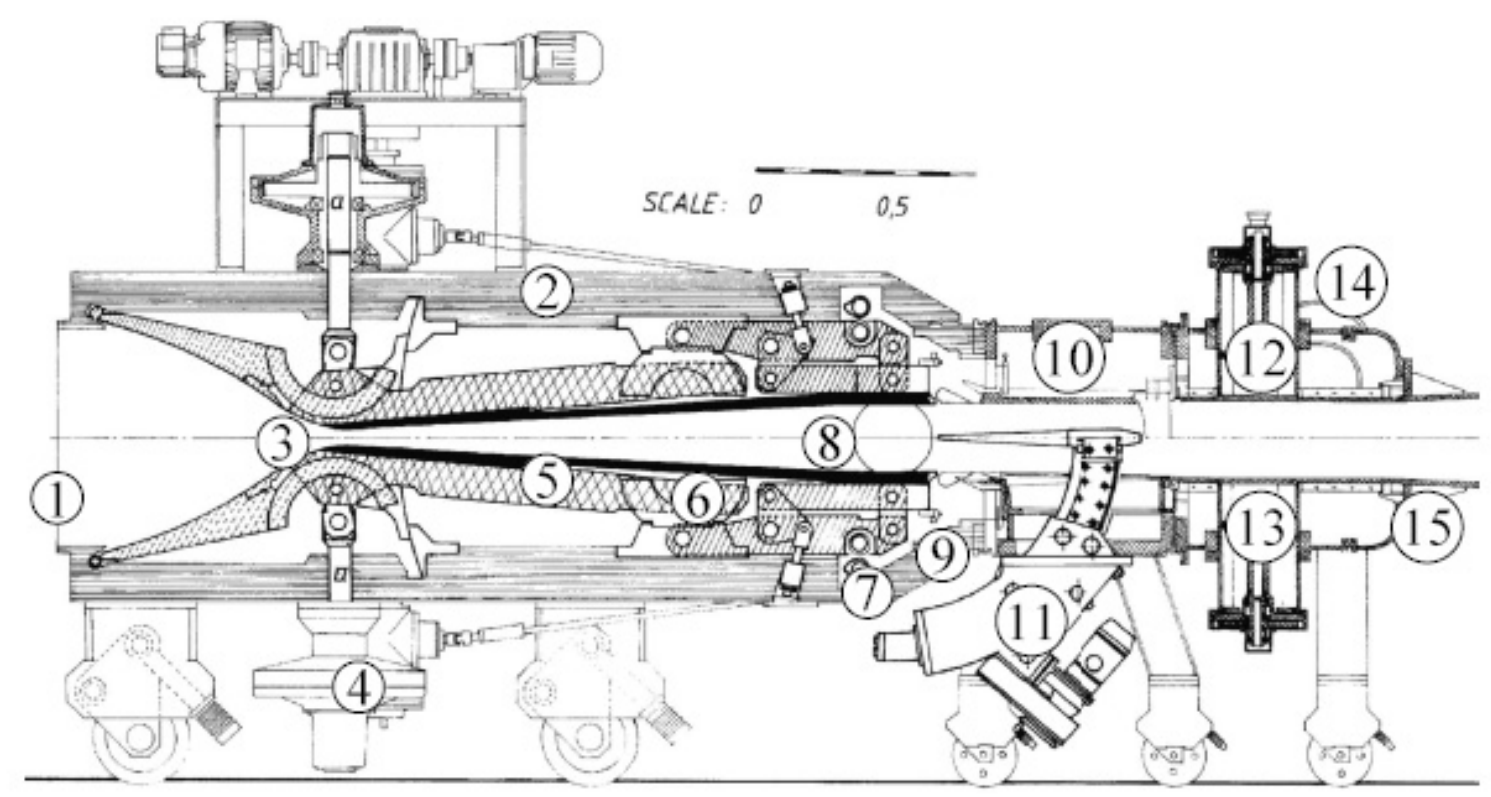

Figure 15: Schematic overview of TST-27

1. Settling chamber

2. Nozzle section

3. Variable nozzle

4. Adjust. screw mech.

5. Semi-flexible wall
6. Node bearing

7. Boundary layer mech.

8. Supersonic test section

9. Rapid clamp coupling

10. Model support section
11. Angle of attack mech.

12. Choke section

13. Choke body

14. Bleed off device

15. Outlet diffuser

To power the tunnel, a $230 \mathrm{~kW}$ computer controlled compressor plant pressurizes a $300 \mathrm{~m}^{3}$ air vessel to approximately $40 \mathrm{bar}$, which corresponds to $14,300 \mathrm{~kg}$ of air. Before the air enters the vessel, it is dried by a high pressure air dryer, resulting in a water content of less than 10 ppm. The wind tunnel can be operated until the pressure in the air tank reaches 20 bar. At 
Mach 2 this results in a measurement time of about 5 minutes. A micron dust filter is installed between the vessel and the tunnel to take out small particles that could damage the measurement equipment.

Downstream of the nozzle and the test section, the tunnel consists of separated modules. These modules are supported on wheels and connected by means of quick-lock couplings. The modules can slide away in downstream direction allowing easy access to the test section. Different modules can be installed, thus enabling the wind tunnel to be operated in different configurations.

\subsection{SWTBLI Experimental Arrangement}

\subsubsection{Incoming boundary layer}

The boundary layer on the side-wall of the wind tunnel was used for the present investigation.

The tunnel operated at a freestream Mach number $\left(\mathrm{M}_{\infty}\right)$ of 1.84 , a total pressure $\left(\mathrm{P}_{0}\right)$ of $2.5 \mathrm{x}$ $10^{5} \mathrm{~N} \cdot \mathrm{m}^{-2}$ and a total temperature $\left(\mathrm{T}_{0}\right)$ of $278 \mathrm{~K}$. These values have been obtained from the tunnels measurement system for supersonic flows which performs temperature measurements in the settling chamber and pressure measurements in both the settling chamber and the test section. The derived static pressure $\left(\mathrm{P}_{\mathrm{e}}\right)$ and static temperature $\left(\mathrm{T}_{\mathrm{e}}\right)$ in the test section were $4.16 \times 104 \mathrm{~N} \cdot \mathrm{m}^{-2}$ and $166 \mathrm{~K}$ respectively. A freestream velocity of $U_{\infty}=483 \mathrm{~m} \cdot \mathrm{s}^{-1}$ was obtained by preliminary PIV measurements. Experimental conditions and boundary layer parameters are listed in table 1.

\begin{tabular}{l|l}
\hline \multicolumn{2}{c}{ Incoming boundary layer properties } \\
\hline $\mathrm{M}_{\infty}$ & $1.84^{\mathrm{a}}$ \\
$\mathrm{U}_{\infty}\left[\mathrm{m} \mathrm{s}^{-1}\right]$ & $483^{\mathrm{b}}$ \\
$\mathrm{P}_{0}\left[\mathrm{Nm}^{-2}\right]$ & $2.52 \times 10^{5 \mathrm{a}}$ \\
$\mathrm{T}_{0}[\mathrm{~K}]$ & $278^{\mathrm{a}}$ \\
$\mathrm{P}_{\mathrm{e}}\left[\mathrm{Nm}^{-2}\right]$ & $4.16 \times 10$ \\
$\mathrm{~T}_{\mathrm{e}}[\mathrm{K}]$ & 166 \\
$\delta_{99}[\mathrm{~mm}]$ & $19^{\mathrm{c}}$ \\
$\delta^{*}[\mathrm{~mm}]$ & $3.8^{\mathrm{c}}$ \\
$\theta[\mathrm{mm}]$ & $1.4^{\mathrm{c}}$ \\
$\mathrm{u}_{\tau}[\mathrm{m} / \mathrm{s}]$ & $17.7^{\mathrm{c}}$ \\
$\mathrm{c}_{\mathrm{f}}$ & $1.6 \times 10^{-3 \mathrm{c}}$ \\
$\mathrm{Re}_{\mathrm{e}} / \mathrm{m}$ & $36.6 \times 10^{6}$ \\
$\operatorname{Re}_{\theta}$ & $5.12 \times 10^{4}$ \\
\hline
\end{tabular}

Table 1: Experimental conditions and incoming boundary layer properties; ${ }^{a}$ measured by the tunnels measurement system; ${ }^{b}$ obtained from preliminary PIV measurements; and ${ }^{c}$ taken from Tuinstra (2005)

Integral boundary layer parameters were taken from Tuinstra (2005), who performed a high resolution planar PIV investigation of a boundary layer in the same facility at a freestream Mach number of 1.86, which is only 0.02 higher than the Mach number in the current experiment. He found that the boundary layer's thickness $\left(99 \%\right.$ of $\left.U_{\infty}\right)$ in the test section was $19 \mathrm{~mm}$, its momentum thickness $(\theta) 1.4 \mathrm{~mm}$ and its displacement thickness $\left(\delta^{*}\right) 3.8 \mathrm{~mm}$. 
Using the van Driest transformation, Tuinstra (2005) determined a skin friction coefficient $\left(\mathrm{c}_{\mathrm{f}}\right)$ of $1.6 \times 10^{-3}$, corresponding to friction velocity $\left(\mathrm{u}_{\tau}\right)$ of $17.7 \mathrm{~m} \cdot \mathrm{s}^{-1}$.

The Reynolds number per meter, $\mathrm{Re}=\mathrm{U}_{\infty} / v_{\infty}$ (where $v_{\infty}$ is the kinematic viscosity in the freestream) was computed to be $36.6 \times 10^{6}$ and that based on the momentum thickness, $\mathrm{Re}=$ $\mathrm{U}_{\infty} \theta / \mathrm{v}_{\infty}=5.12 \times 10^{4}$.

\subsubsection{Shock generator}

A half-wedge shaped model that deflects the flow by $10^{\circ}$ degrees was used as a shock generator. It had a length of $70 \mathrm{~mm}$ and a width of $180 \mathrm{~mm}$, spanning approximately two thirds of the test section. The model was placed in the middle of the tunnel by means of a sting. Using charts of the Ames research staff (1953), a shock wave angle of $43^{\circ}$ was predicted. The expansion fan resulting from the shoulder of the model does not influence the interaction within the present measurement domain.

\subsubsection{Micro-Ramps}

Micro-ramps were used in the experiments to perturb the incoming boundary layer. These SBVGs consisted of small triangular ramps machined out of aluminium and were attached to the wall using double-sided tape. Each micro-ramps had a hole with screw thread $(\varnothing=3 \mathrm{~mm})$ close to its highest point to facilitate the removal of the ramp from the wall.

The dimensions of the micro-ramps and their spanwise packing were scaled to their height according recommendations by Anderson et al. (2006) for minimizing the shape factor downstream of the interaction. The height was chosen to be $4 \mathrm{~mm}(\mathrm{~h} / \delta=0.2)$ and such that the effect of multiple micro-ramps could be seen within the field of view. The resulting size and shape of the micro-ramps is shown in (figure 16)

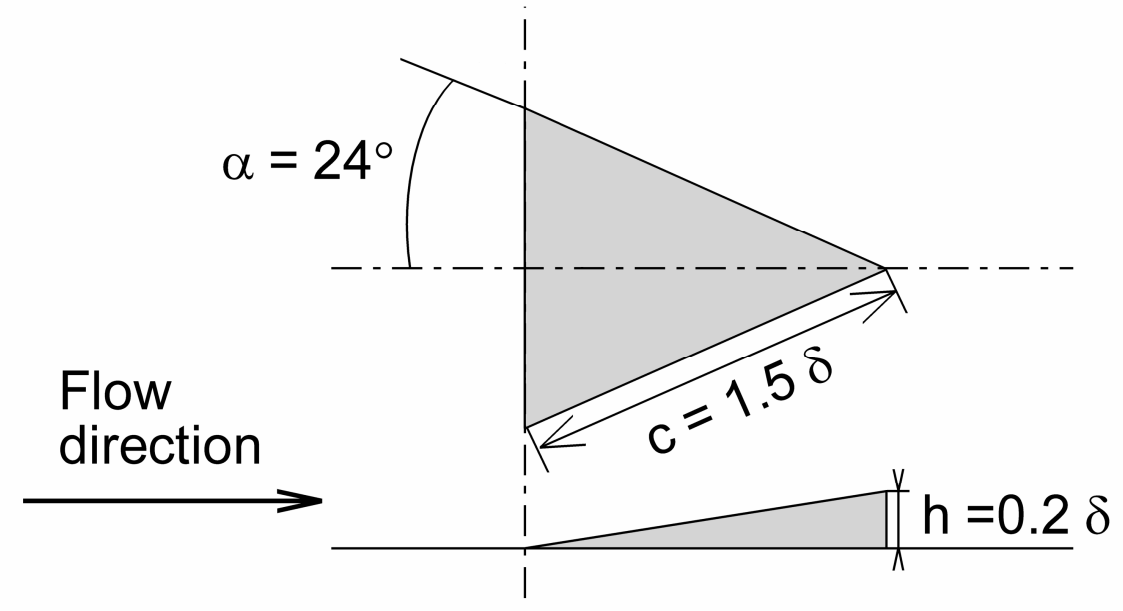

Figure 16: dimensions of the micro-ramp

The ramps were placed $20 \mathrm{~cm}(10 \delta)$ upstream of the origin of the interaction, which was defined on the cross-section of the centreline of the tunnel wall and the line where the incident shock would impinge on the wall in absence of a boundary layer. This generator-to-shock spacing was chosen based on a range of effectiveness (see section 2.7) reported by Pitt Ford and Babinsky (2007) for similarly shaped micro-ramps with heights ranging from $\mathrm{h} / \delta=0.25$ 0.75 for a freestream Mach number of 2.5 and a Reynolds number of $2.46 \times 10^{6}$ based on the boundary layer's momentum thickness. The location of the micro-ramps in the present investigation is located approximately in the middle of this range of effectiveness whether it is 
scaled by the boundary layer thickness $\delta$, the incompressible displacement thickness $\delta^{*}{ }_{\mathrm{i}}$ or the incompressible momentum thickness $\theta_{\mathrm{i}}$.

Two configurations of micro-ramps were used: a single row of five micro-ramps (figure 17) and staggered configuration (see figure 18).

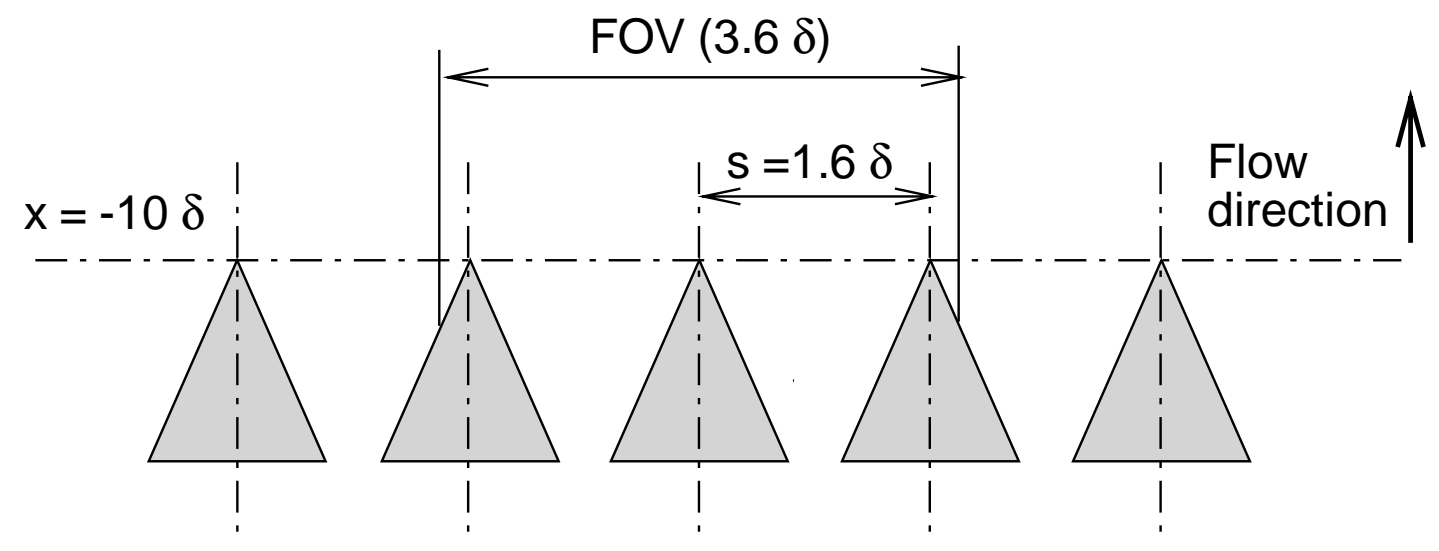

Figure 17: schematic of the single row of micro-ramps

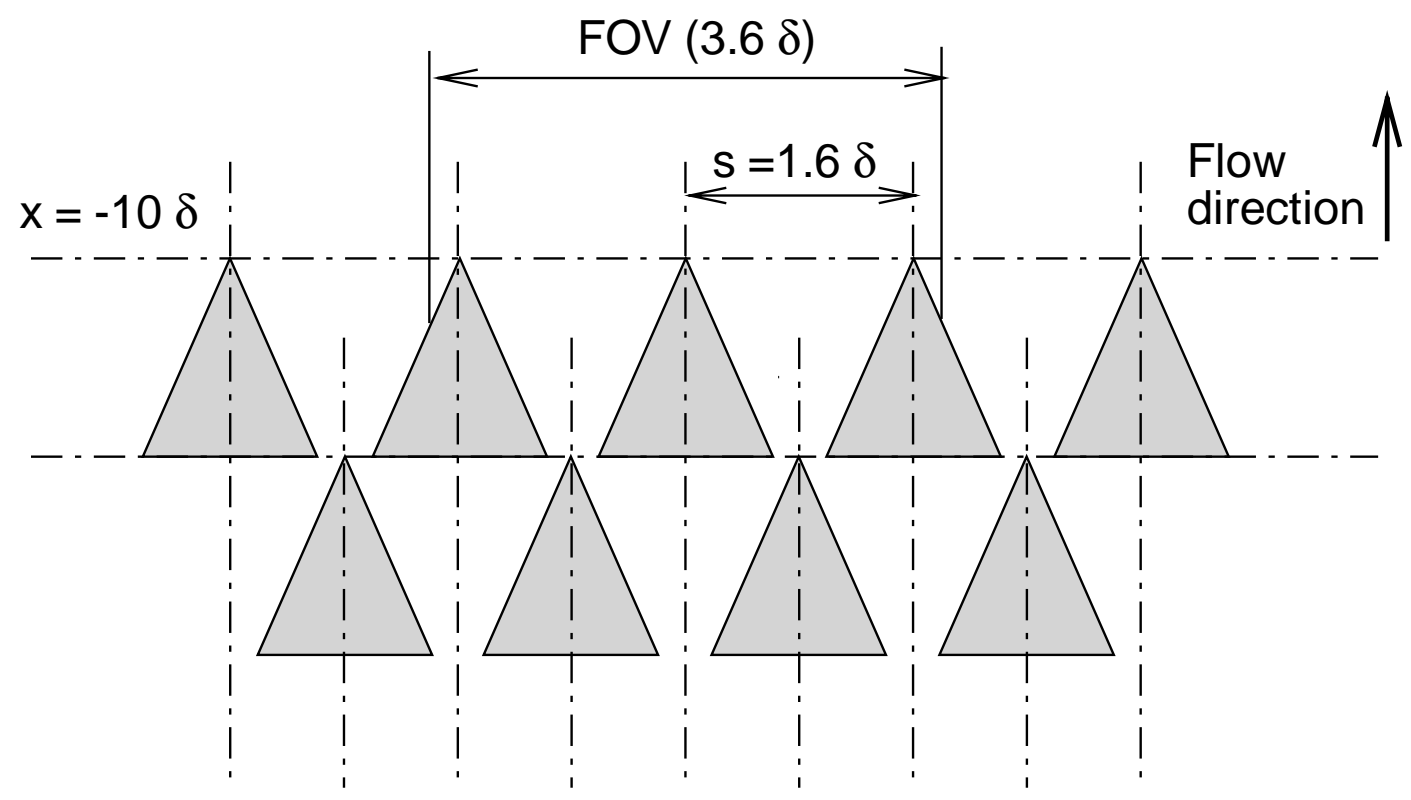

Figure 18: schematic of the staggered array of micro-ramps

\subsection{Oil-Flow Visualization}

Oil-flow visualization was used to investigate the flow organization at the wall. The technique is widely used surface flow visualization method. It employs a dye dispersed in oil that is smeared on a surface. Due to the viscous action of the flow close to the surface, the oil moves slowly in the local flow direction, allowing global acquisition of near-wall velocity directional information. In the present experiment, the following mixture was used for the visualization (in volumetric parts): 18 Titanium-dioxide, 18 Kaoline, 1 Tween 80 and 18 Petroleum. The thickness of the oil-film is an important experimental parameter: if the thickness of the film is too thick, oil patterns will be hard to identify. Also the viscosity of the oil is essential for 
obtaining distinct oil patterns: a mixture that has a very low viscosity will not be carried away by the flow, while a mixture with too high a viscosity will be blown away during the start-up of the tunnel or the pattern may become obscured during shutdown. From the above it follows that the quality of the acquired oil pattern is very dependent on run time. This has to be long enough to allow the oil to form a steady state pattern, excluding start-up effects, and at the same time short enough to preclude excessive oil-accumulation. In the present experiment the viscosity of the flow was increased by adding a few drops of oleic acid. In order to improve the visibility of the flow pattern, black adhesive foil was put on the bottom of the test-section, where the oil was applied.

\subsection{Particle image velocimetry}

Stereo-PIV was used to obtain velocity fields at $0.1 \delta$ and $0.6 \delta$ away from the wall. In this section the basic principles of this technique will be discussed together with a description of the used setup for the present experiment.

\subsubsection{Working principles}

In order to perform PIV measurements tracer particles need to be added to the flow. The flow seeded with tracer particles is illuminated twice within a short interval by laser pulses that have been shaped into a sheet. The interval between the two pulses needs to be relatively short compared to the relevant time scales in the flow. The two exposures are recorded by one or more cameras. From the known time difference between the two illuminations and the measured displacement of the particles a velocity field can subsequently be calculated. A schematic overview of a typical PIV setup is shown in figure 19. In the present experiment recording is performed by two cameras placed at angles to the laser sheet. This variant of PIV is denoted as stereo-PIV and allows the measurement of particle displacement perpendicular to the laser sheet and hence out-of-plane velocity measurements.

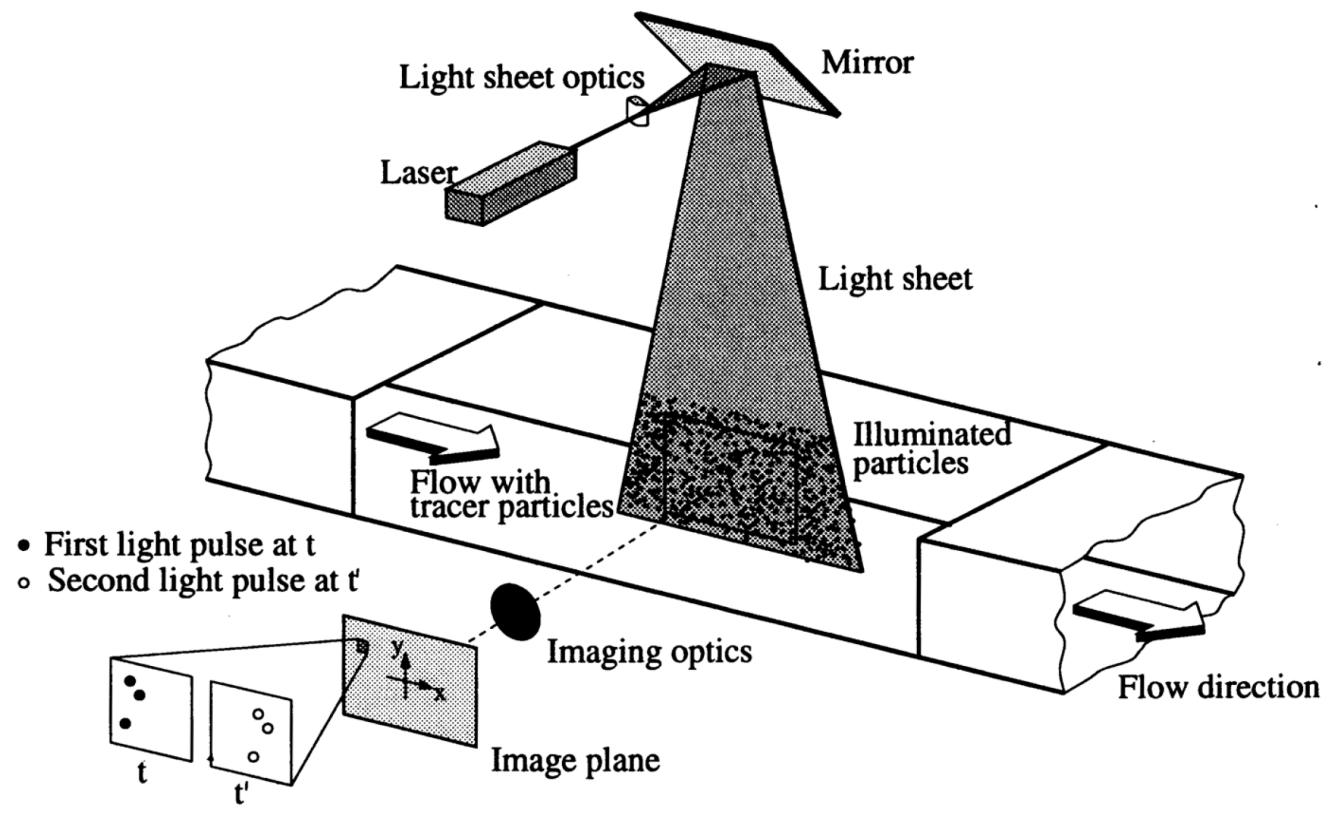

Figure 19: schematic overview of a PIV setup (Raffel et al. 1998)

The processing of the recorded images involves the use of advance analysis software. The procedure that is carried out to obtain vector fields generally consist of the following steps 
which are illustrated in figure 20: first the images are divided in small regions called interrogation windows. The size of these windows should be such that all particles in it move homogenously. For each interrogation window the displacement of the particles is now calculated by means of a correlation function. Knowing the displacement of the particles, the magnification factor and the time separation, a velocity vector can be determined for each window.
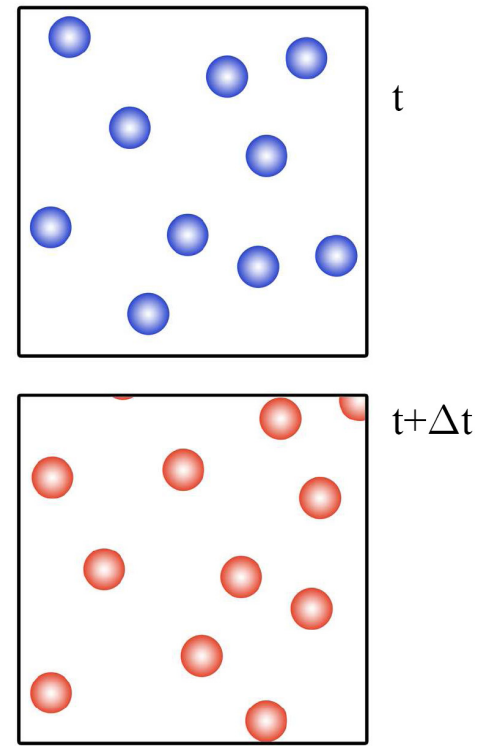

One window out of the recorded images
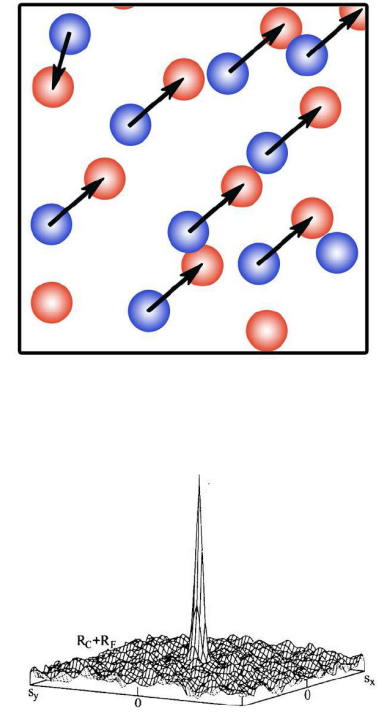

Cross-correlation and correlation map

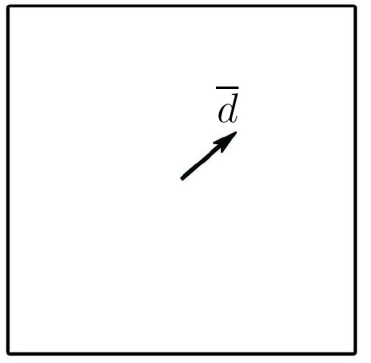

$$
\bar{V}=\frac{\bar{d}}{\Delta t}
$$

Velocity vector

Figure 20: basic analysis principle (Kuik 2007)

Since PIV is an optical technique, no probes that may disturb the flow need to be used in the flow region under investigation. This gives PIV a clear advantage over intrusive techniques that for example use pressure tubes or hot-wires. Another advantage of PIV is that it allows instantaneous velocity measurements of a large part of the flow. Although the spatial resolution of PIV is large, the temporal resolution in terms of frame rate of the recordings is limited by the repetition rate of lasers and the recording rate of cameras. Another drawback of PIV is that it only performs indirect measurements. Similar to laser Doppler velocimetry, PIV does not directly measure the velocity of the flow. Instead it measures the velocity of particles that are assumed to follow the flow faithfully. Because of particle inertial lag significant errors may however occur if the flow contains large velocity gradients and strong shocks (Scarano and Van Oudheusden 2003).

\subsubsection{Flow seeding}

The assessment particle motion relative to the fluid motion is an important issue in PIV investigations of high-speed compressible flows. With the following assumptions:

- particles are spherical

- diameter is much smaller than the turbulence length scale of interest

- low particle concentration

- particle density is much higher than surrounding fluid density

- forces like buoyancy, added mass and Basset history integral are negligible 
- Stokes' law can be applied

The equation of motion of a particle entrained in a steady flow is reduced to (Melling 1997):

$$
\frac{d \vec{U}_{p}}{d t}=\frac{\vec{U}_{p}-\vec{U}}{\tau_{p}}
$$

Where $U_{p}$ is the particle velocity and $U$ is the fluid velocity. The particle relaxation time $\tau_{\mathrm{p}}$ is a measure for how well the particles follow the flow and can be obtained from (Schrijer and Scarano 2007):

$$
\tau_{p}=\frac{4}{3} \frac{\rho_{p} d_{p}^{2}}{c_{d} \operatorname{Re}_{d} \mu_{f}}
$$

Where $\rho_{p}$ is the particle density, $d_{p}$ is the particle diameter, $c_{d}$ is the particle drag coefficient, $\mathrm{Re}_{\mathrm{d}}$ is the particle Reynolds number based on the slip velocity and the particle diameter and $\mu_{\mathrm{f}}$ is the fluid viscosity. From the expression it can be seen that smaller particles follow the flow more faithfully than larger particles. The particle relaxation time can experimentally be determined by measuring the responds of a particle to a step-input. This is achieved by measuring the particle motion through a shock. A measure for the fidelity of flow tracers in turbulent flow is the Stokes number St, which is defined as the ratio between the particle relaxation time $\tau_{\mathrm{p}}$ and a time scale of the flow $\tau_{\mathrm{f}}$. For accurate tracking at the time scale $\tau_{\mathrm{f}}$, it is necessary that $\mathrm{St}<<1$.

It is remarked that although from a fluid mechanical point of view small particles are needed, large particles are desired to obtain sufficient light scattering. In order to keep the particles as small as possible, particles with proper scattering properties are used in combination with high-power lasers. Apart from particle size and light intensity, the amount of light scattering that can observed, depends on the shape and orientation of the particle, the ratio of the refractive index of the particle to that of its environment, the observation angle and the polarization of the light (Raffel et al. 1998).

In the present experiment titanium dioxide $\left(\mathrm{TiO}_{2}\right)$ particles of the type Dupont R-931 with nominal diameter of $0.55 \mu \mathrm{m}$ were used as tracer particles. The density of the seeding as reported by the manufacturer is $3.6 \times 10^{3} \mathrm{~kg} / \mathrm{m}^{3}$. It should be noted that the effective particle size can differ significantly from the nominal particle size due to particle agglomeration. Schrijer et al (2006) quantified this effect by performing an electron scan of titanium dioxide particles with a nominal particle size of $50 \mathrm{~nm}$ and found an effective particle size that was 8 times larger. The extent of the agglomeration is dependent of many factors such as storage conditions and the distribution procedure.

Schrijer and Scarano (2007) performed particle response assessments for different types of titanium dioxide powder. For the present seeding they inferred a particle relaxation length $\left(\xi_{\mathrm{p}}\right)$ of $0.95 \mathrm{~mm}$ and a particle relaxation time $\left(\tau_{\mathrm{p}}\right)$ of $3.5 \mu \mathrm{s}$, corresponding to a frequency responds $\left(f_{p}\right)$ of $286 \mathrm{kHz}$. Based on an outer time scale of $\delta / \mathrm{U}_{\infty}, \tau_{\mathrm{f}}$ is $42 \mu \mathrm{s}$. This gives a Stokes number of 0.08 , which is of the same order as reported by Humble et al. (2007)

A high pressure cyclone aerosol generator was used to disperse the powder in air and to control the amount of seeded flow that was injected in the tunnel (see figure 21). This device works in the following way: the air that enters through the primary air inlet creates a cyclone 
in the pod causing turbulent mixing of the air with the seeding. The formed aerosol leaves the pod and is transported to the wind tunnel. Air can be added through the secondary air inlet to further increase the mass and velocity of the seeded air entering the tunnel.

Air pressurized at $10 \times 10^{5} \mathrm{~Pa}$ was connected to the primary and secondary inlets. The amount of air that entered through the inlets and the outlet could be controlled by valves A, B and C. In order to obtain adequate seeding, valves $\mathrm{B}$ and $\mathrm{C}$ were both fully opened. Valve A was opened so far that the manometer directly behind it indicated $6 \times 10^{5} \mathrm{~Pa}$. The valve tuned online after the start-up of the wind tunnel. Recordings were not started before adequate seeding was obtained.

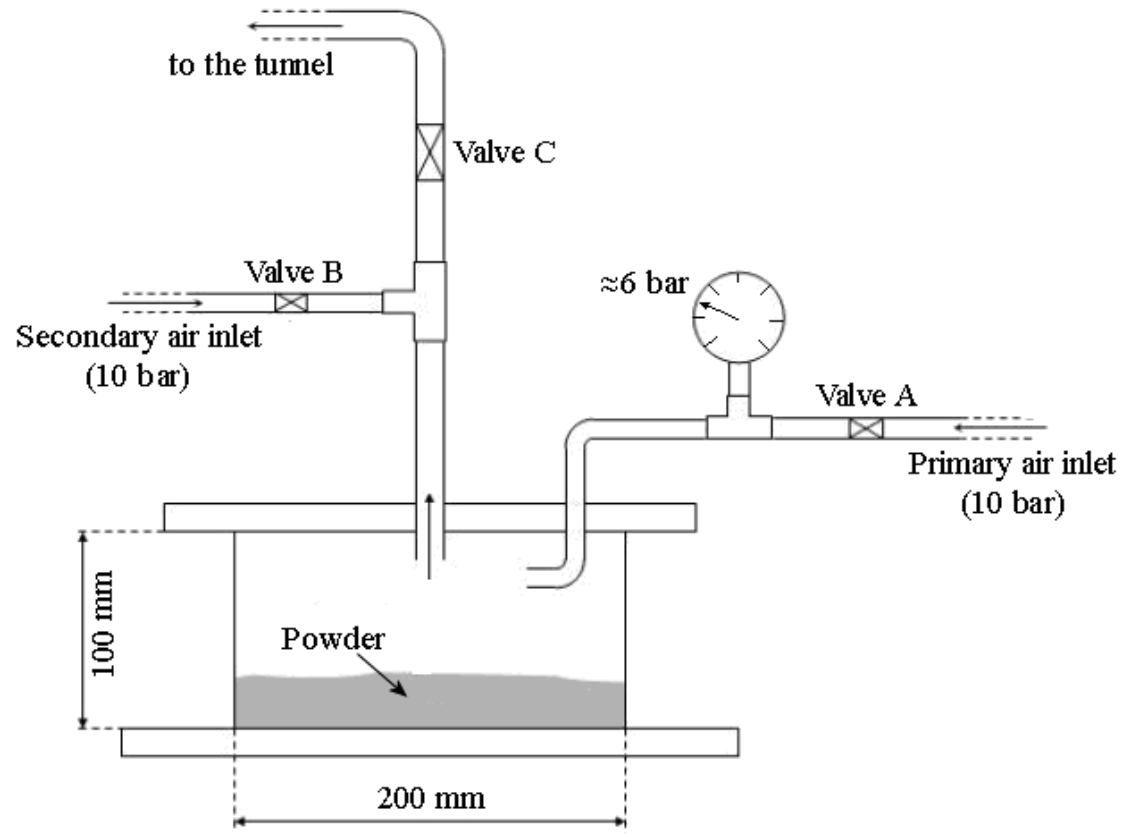

Figure 21: schematic of the seeding generator (adjusted from Tuinstra 2005)

The seeded flow was brought into the tunnel by a probe that was put in the side of the settling chamber. At the test section seeded flow was present within a slightly meandering streamtube spanning approximately $4 \delta$ at $y / \delta=0.6$, where y denotes the distance from the wall. Although the concentration of the seeding near the sides of the streamtube could vary considerably because of the meandering motion, it was in general found to be significantly lower than in the middle part At $y / \delta=0.1$ the seeding was more spread out presumably because of the higher turbulence levels.

\subsubsection{Illumination}

The seeded flow was illuminated by a Spectra-Physics Quanta Ray PIV-400 double-cavity Nd:Yag laser. This laser is capable of firing sets of two pulses with a wavelength of $532 \mathrm{~nm}$ at $10 \mathrm{~Hz}$. Each individual pulse has a maximum energy of $400 \mathrm{~mJ}$ and a duration of $6 \mathrm{~ns}$. The pulses leave the laser and enter a probe in which it is deflected and shaped into a sheet. The probe was turned $6^{\circ}$ along its length axis in order to completely illuminate the field of view (see figure 22). The edges of the laser sheet in reality are not as sharply defined as in figure 22 . 


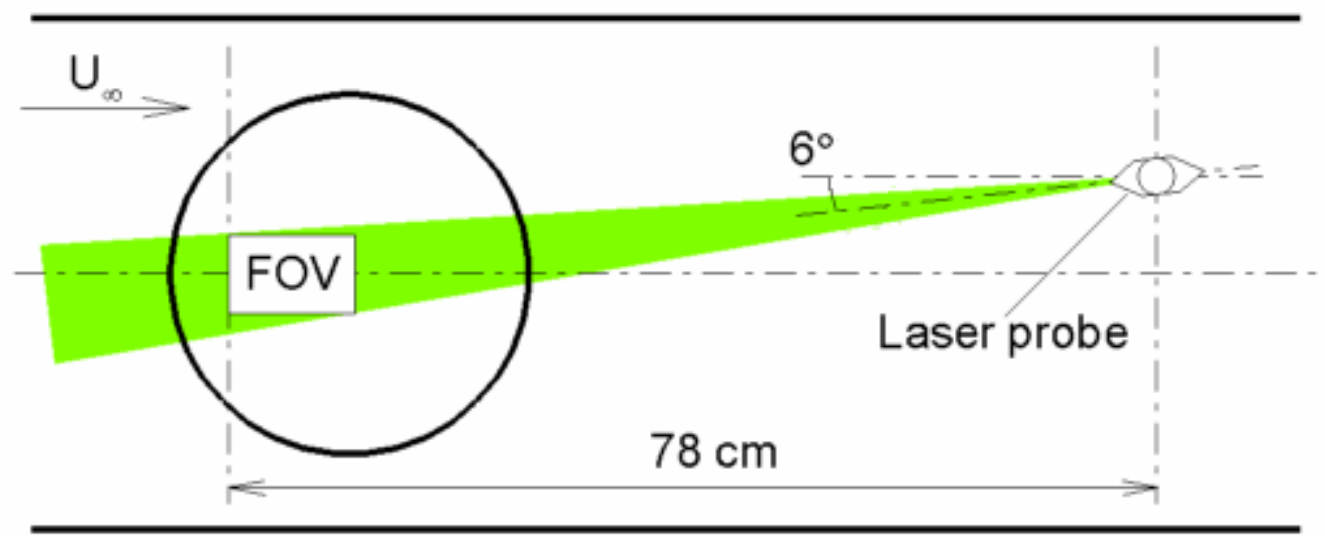

Figure 22: laser sheet position (side view)

Because of size and placement of the probe, the sheet needed to be at a slight angle to illuminate the seeding close to the wall. In order to perform measurements as close to the wall as possible, this angle has been chosen such that the sheet hits the window just upstream of the field of view for measurements of the lower boundary level $(\mathrm{y} / \delta=0.1)$. For the measurements further away from the wall $(\mathrm{y} / \delta=0.6)$, the laser probe was translated $10 \mathrm{~mm}$.

In order to minimize the thickness of the laser sheet, it was set to converge towards the centre of the field of view. Because the laser caused too many reflections on the window, the sides of the beam were cut of before entering the probe. The cutting was done in such a way, that the side of the laser sheet closest to the window touches the window just upstream of the field of view. The combined effect of the optics inside probe and the cutting of the beam resulted in a laser sheet thickness of 1.0-1.5 mm, depending on the streamwise location.

\subsubsection{Recording}

Images were recorded by a PCO Sensicam QE CCD camera and a LaVision Imager Intense CCD camera which have both have a dynamic range of 12 bits and a 1376x1040 pixel-sized sensor with a pixel pitch of $6.45 \mu \mathrm{m}$. The cameras have a frame straddling architecture allowing frame straddling for laser pulse separations down to $0.5 \mu \mathrm{s}$. Each camera was equipped with a Nikon $60 \mathrm{~mm}$ focal objective and a daylight filter transmitting only light with a wavelength of $532 \mathrm{~nm}$. It was found that by choosing an $f$-number of 8 , both light intensity and diffraction spot size were satisfactory.

The cameras were placed in a stereo configuration to also be able to measure the out-of-plane velocity component and to allow self-calibration using recorded images. This refines the manual calibration which is needed to define the viewing plane such that it coincides with the laser sheet. The angle between both cameras was approximately $35^{\circ}$ and the recording distance was $70 \mathrm{~cm}$. This resulted in a field of view of $101 \times 73 \mathrm{~mm}(5.6 \delta \times 3.8 \delta)$ and a digital resolution of $13.6 \mathrm{pix} / \mathrm{mm}$. The field of view was positioned such that the upsteam boundary layer, the interaction and part of the recovery are visible both at $y / \delta=0.1$ and $y / \delta=0.6$.

When a camera is placed at an angle with respect to the subject plane, it may become impossible to get the complete field of view within the focal depth. In the current experiment, this problem was avoided by placing Scheimpflug adapters between the lenses and the cameras. A Scheimpflug adapter makes it possible to rotate the lens plane with respect to the image plane. When this is done correctly, the focal plane becomes parallel to the subject plane. 
Each camera was mounted on a holder that was used to accurately control the sideway position of the camera. This holder was attached to a slide with which the camera's back and forth position could be controlled. The slide was screwed on a accurately levelled heavy tripod, which was used to control the height of the camera.

Both the cameras and the laser were connected to a computer with a LaVision programmable timing unit (PTU) to provide for the digital synchronization. DaVis 7.1 software was used to control the PTU. The image acquisition system was set to record at a frequency of $5 \mathrm{~Hz}$ with a time separation between two subsequent exposures of $2 \mu \mathrm{s}$, which resulted in pixel displacement of about 12 pixels for a typical particle in the undisturbed boundary layer.

\begin{tabular}{l|l}
\hline \multicolumn{2}{c}{ PIV recording parameters } \\
\hline Field of view, W x H & $101 \mathrm{~mm} \times 73 \mathrm{~mm}$ \\
Final interrogation & $(5.4 \delta \times 4.0 \delta)$ \\
window size & $32 \times 32$ pixels \\
Overlap factor & $75 \%$ \\
Digital resolution & 13.6 pixels $/ \mathrm{mm}$ \\
Vector spacing & $0.6 \mathrm{~mm}$ \\
Recording lens & $\mathrm{f}=60 \mathrm{~mm}$ \\
f-number & $\mathrm{f}_{\#}=8$ \\
Laser pulse separation & $2 \mu \mathrm{s}$ \\
Ensemble size & 340 \\
\hline
\end{tabular}

Table 2: PIV recording parameters

PIV recordings were processed with DaVis 7.4 software. To remove the effect of broken pixels on the CCD and to minimize unwanted reflections, the minimum value for every pixel in a set of recordings was determined and subtracted from each image in the set. Velocity vector fields were obtained by cross-correlation of the images based on a fast Fourier transform-based algorithm using a multi-grid approach, with a final window size of $32 \times 32$ pixels with a $75 \%$ overlap. This leaded to one vector per $8 \times 8$ pixels or $0.6 \times 0.6 \mathrm{~mm}$. Spurious vectors were removed using the median test. The average signal-to-noise, defined as the ratio between the first and second correlation peak typically had a value in the range 1.5-2.0. The final dataset consists of 340 instantaneous vector fields for each configuration. Vectors were corrected for a laser sheet angle of $1^{\circ}$ with respect to the wall. This angle was visually inferred from photos of the laser sheet.

\subsection{Overview of the setup}

In order to summarize the discussion on the experimental arrangement, the complete setup is shown in figure 23.

The PIV setup used to perform measurements at $y / \delta=0.1$ is depicted in this figure as well. Micro-ramps can be seen to be placed inside the boundary layer, which interacts with the shock reflection more downstream. Experiments have been performed with and without the shock generator and with and without the micro-ramps in order to investigate both the SWTBLIs and the boundary layers. 


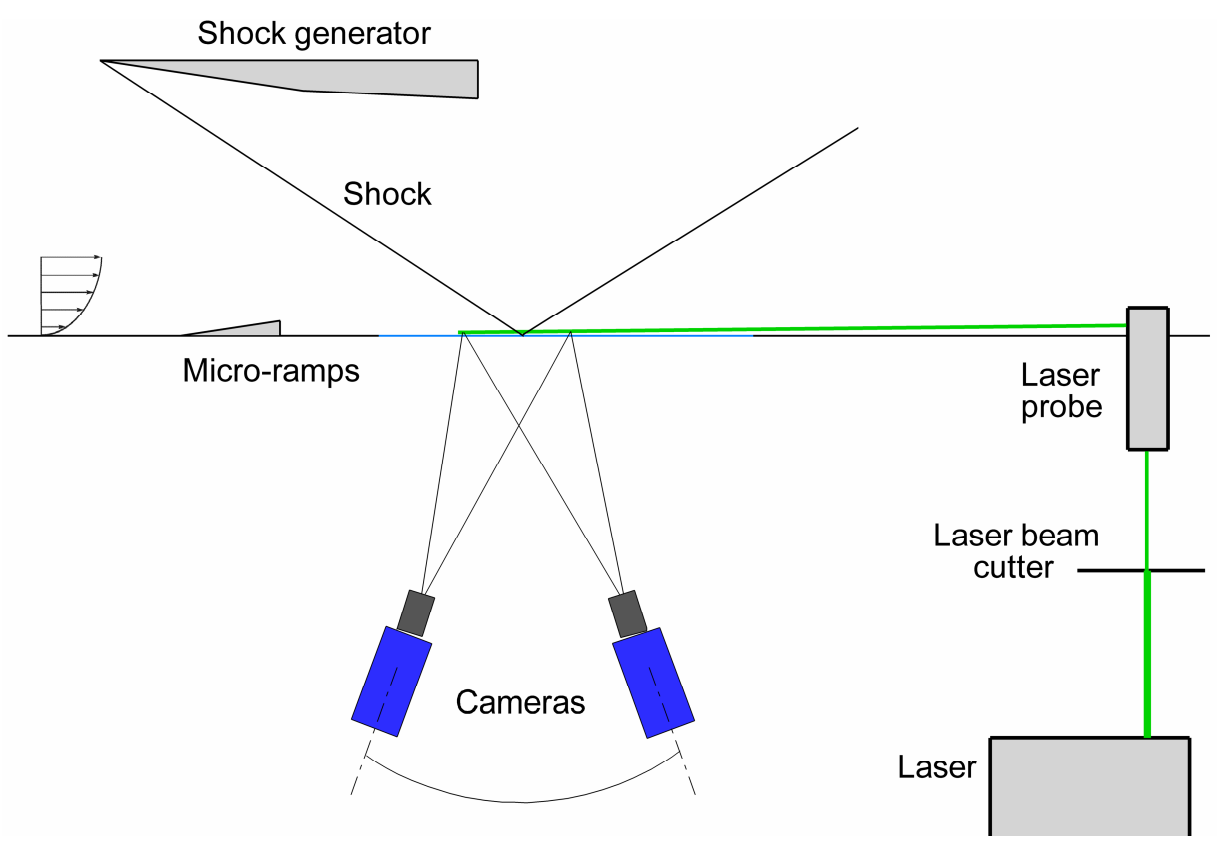

Figure 23: schematic of the experimental setup (top view); not drawn on scale.

\subsection{Uncertainty analysis}

Now the principles of the experimental techniques and the used arrangements have been discussed, the uncertainty involved in the measurements can be looked upon. Throughout the discussion, the streamwise velocity component $(\mathrm{u})$ will be taken as example.

Mean and root-mean-square (RMS) velocities were determined using the following well known expressions:

$$
\begin{gathered}
\bar{u}(\mathbf{x})=\frac{1}{N} \sum_{n=1}^{N} u\left(\mathbf{x}, t_{n}\right) \\
\overline{u^{\prime 2}}(\mathbf{x})=\frac{1}{N} \sum_{n=1}^{N}\left[u\left(\mathbf{x}, t_{n}\right)-\bar{u}(\mathbf{x})\right]
\end{gathered}
$$

where $\mathrm{N}$ is the ensemble size. The RMS uncertainty $\varepsilon$ induced by the finite number of samples for the mean and RMS velocities can be quantified with the following expressions (Humble 2008):

$$
\begin{gathered}
\varepsilon_{\bar{u}}=\frac{\sqrt{\overline{u^{\prime 2}}}}{\sqrt{N}} \\
\varepsilon_{\sqrt{u^{\prime 2}}}=\varepsilon_{<u^{\prime}>}=\frac{\sqrt{\overline{u^{\prime 2}}}}{\sqrt{2 N}}
\end{gathered}
$$

Apart from these uncertainties, each instantaneous velocity and velocity gradient has an inherent RMS uncertainty; $\varepsilon_{\mathrm{u}}$ and $\varepsilon_{\partial \mathrm{u} / \partial \mathrm{x}}$ respectively, which depend on a wide variety of parameters such as seeding quality, laser time-separation, window interrogation size etc. 
(Raffel et al. 2007). First the RMS uncertainty of the (reciprocal) spatial resolution will be considered. Since stereo-PIV is used, both the in-plane and out-of-plane velocity component must be looked upon. For this reason two different spatial resolutions are defined, the in-plane spatial resolution and the projected spatial resolution that is appropriate for the out-of-plane velocity component:

$$
\begin{gathered}
\kappa_{\text {in-plane }}=\frac{l_{c}}{n_{c}}=\frac{L_{x}}{n_{x}} \\
\kappa_{\text {out-of-plane }}=\kappa_{\text {in-plane }} \sin (\beta)
\end{gathered}
$$

where $1_{c}$ is the distance between the calibration plate dots corresponding to $n_{c}$ pixels, $L_{x}$ is the length of the field of view corresponding to $\mathrm{n}_{\mathrm{x}}$ pixels and $\beta$ is the angle between the CCD-chip and the measurement plane. With the assumption that different parameters that give rise to uncertainty are uncorrelated, Humble (2008) derived the following expression for the uncertainty of the appropriate (reciprocal) spatial resolution $\varepsilon_{\mathrm{\kappa}}$ for self-calibrated PIV recordings:

$$
\varepsilon_{\kappa}=\sqrt{\left(\frac{l_{c}}{n_{c}^{2}} \varepsilon_{n_{c}}\right)^{2}}=\sqrt{\left(\frac{\kappa}{n_{c}} \varepsilon_{n_{c}}\right)^{2}}
$$

where $\varepsilon_{n_{c}}$ is the RMS uncertainty of $\mathrm{n}_{\mathrm{c}}$ which can be based on the self-calibration disparity map and $\kappa$ is the appropriate spatial resolution. Following Humble (2008) the RMS uncertainty of the particle displacement $\varepsilon_{\Delta \mathrm{s}}$ and of the vector spacing $\varepsilon_{\Delta \mathrm{x}}$ can now be estimated using:

and,

$$
\varepsilon_{\Delta s}=\sqrt{\left(\kappa \varepsilon_{c c}\right)^{2}+\left(\frac{\Delta s}{\kappa} \varepsilon_{\kappa}\right)^{2}}
$$

$$
\varepsilon_{\Delta x}=W_{s} \frac{(100-O F)}{100} \varepsilon_{\kappa_{\text {in-plane }}}
$$

where $\varepsilon_{\mathrm{cc}}$ is the RMS uncertainty in the digital cross-correlation, $\mathrm{W}_{\mathrm{s}}$ is the size of the interrogation window size in pixels and OF is the overlap factor in percentage. Note that $\Delta \mathrm{s}$ can denote both the in-plane and the out-of-plane component of the particle displacement. Assuming that $\varepsilon_{\mathrm{u}}$ is uniform throughout the domain, the RMS uncertainties of the instantaneous velocity $\varepsilon_{\mathrm{u}}$ and velocity gradient $\varepsilon_{\partial \mathrm{u} / \partial \mathrm{x}}$ can now be approximately be determined with the following expressions (Humble 2008):

$$
\begin{aligned}
\varepsilon_{u} & =\sqrt{\left(\frac{1}{\Delta t} \varepsilon_{\Delta s}\right)^{2}+\left(\frac{\Delta s}{\Delta t^{2}} \varepsilon_{\Delta t}\right)^{2}} \\
\varepsilon_{\partial u / \partial x} & =\sqrt{2\left(\frac{1}{2 \Delta x} \varepsilon_{u}\right)^{2}+\left(\frac{\partial u}{\partial x} \frac{\varepsilon_{\Delta x}}{\Delta x}\right)^{2}}
\end{aligned}
$$

where $\Delta \mathrm{t}$ and $\varepsilon_{\Delta \mathrm{t}}$ are the laser pulse time separation and its associated RMS uncertainty. 
All RMS uncertainties considered above have been calculated or taken from literature and are summarized on the next page in table 3 .

\begin{tabular}{|c|c|}
\hline \multicolumn{2}{|c|}{ Summary of measurement uncertainties } \\
\hline $\mathrm{U}_{\infty}$ & $483 \mathrm{~ms}^{-1}$ \\
\hline ensemble size $\mathrm{N}$ & 340 \\
\hline $\mathrm{L}_{\mathrm{x}}$ & $101 \mathrm{~mm}$ \\
\hline $\mathrm{n}_{\mathrm{x}}$ & 1376 pixels \\
\hline$\beta$ & $17.5^{\circ}$ \\
\hline$\kappa_{\text {in-plane }}$ & $0.073 \mathrm{~mm} /$ pixel \\
\hline$\kappa_{\text {out-of-plane }}$ & $0.022^{\mathrm{a}} \mathrm{mm} / \mathrm{pixel}$ \\
\hline $1_{c}$ & $10 \mathrm{~mm}$ \\
\hline $\mathrm{n}_{\mathrm{c}}$ & 137 pixels \\
\hline $\mathrm{W}_{\mathrm{s}}$ & 32 pixels \\
\hline OF & $75 \%$ \\
\hline$\varepsilon_{\mathrm{cc}}$ & 0.1 pixels $^{\mathrm{b}}$ \\
\hline$\varepsilon_{\mathrm{nc}}$ & 0.1 pixels $^{c}$ \\
\hline$\varepsilon_{\mathrm{K}} / \kappa($ in- and out-of-plane) & $<0.1 \%$ \\
\hline$\Delta \mathrm{s}_{\text {in-plane }}$ & $0.9 \mathrm{~mm}^{d}$ \\
\hline$\Delta \mathrm{s}_{\text {out-of-plane }}$ & $0.1 \mathrm{~mm}^{e}$ \\
\hline$\Delta \mathrm{t}$ & $2 \mu \mathrm{s}$ \\
\hline$\Delta \mathrm{x}$ & $0.6 \mathrm{~mm}$ \\
\hline$\varepsilon_{\Delta s} / \Delta \mathrm{s}$ (in-plane) & $0.8 \%$ \\
\hline$\varepsilon_{\Delta s} / \Delta \mathrm{s}$ (out-of-plane) & $1.4 \%$ \\
\hline$\varepsilon_{\Delta t} / \Delta \mathrm{t}$ & $0.4 \%{ }^{\mathrm{f}}$ \\
\hline$\varepsilon_{\Delta x} / \Delta \mathrm{x}$ & $<0.1 \%$ \\
\hline$\varepsilon_{\overline{\mathrm{u}}} / \mathrm{U}_{\infty}$ & $1.1 \%^{\mathrm{g}}$ \\
\hline$\varepsilon_{\left\langle u^{\prime}>\right.} / U_{\infty}$ & $0.8 \%{ }^{\mathrm{h}}$ \\
\hline$\varepsilon_{\mathrm{u}} / \mathrm{U}_{\infty}$ & $0.9 \%$ \\
\hline$\varepsilon_{\mathrm{v}} / \mathrm{U}_{\infty}$ & $0.3 \%$ \\
\hline$\varepsilon_{\partial \mathrm{u} / \partial \mathrm{x}} /\left(\mathrm{U}_{\infty} / \delta\right)$ & $20 \%{ }^{\mathrm{i}}$ \\
\hline
\end{tabular}

Table 3: summary of measurement uncertainties

\footnotetext{
${ }^{a}$ based on maximum flow angle w.r.t. measurement plane

${ }^{\mathrm{b}}$ see Raffel et al. (2007)

${ }^{\mathrm{c}}$ based on self-calibration disparity map (Raffel et al. 2007)

${ }^{\mathrm{d}}$ based on maximum in-plane velocity

${ }^{\mathrm{e}}$ based on maximum out-of-plane velocity

${ }^{\mathrm{f}}$ based on laser pulse width

${ }^{\mathrm{g}}$ based on maximum RMS

${ }^{\mathrm{h}}$ based on maximum RMS

${ }^{\mathrm{i}}$ based on maximum $\partial \mathrm{u} / \partial \mathrm{x}$
} 
EFFECTS OF MICRO-RAMPS ON A SHOCK WAVE BOUNDARY LAYER INTERACTION 


\section{Chapter 4: Oil-Flow Visualization Results and Discussion}

\subsection{Undisturbed interaction}

An oil-flow pattern of the undisturbed interaction is depicted in figure 24, in order to show the basic features of flow at the wall's surface. The flow direction is from top till bottom and is shown in perspective view. The PIV measurement domain is enclosed by the solid white line.

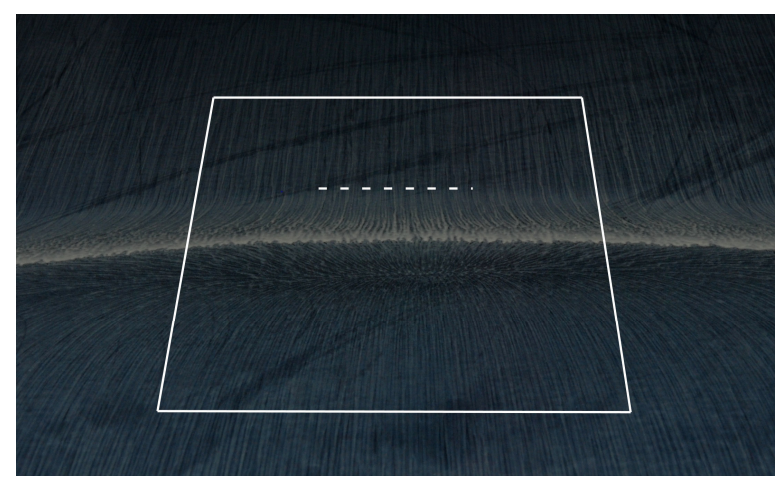

Figure 24: oil-flow visualizations of undisturbed interaction; the (projected) PIV measurement domain $(5.4 \delta \times 3.6 \delta)$ is enclosed by the solid line; the dashed line indicates onset of the interaction

The location of impinging shock wave is indicated by the most upstream position of oilaccumulation (Doerffer and Swaba 2004). In order to facilitate interpreting this position, it marked by the dashed line in the middle of the field of view. The most downstream position of oil-accumulation could indicate the position of flow separation (Green 1970; Doerffer and Swaba 2004). Farther downstream a region is apparent from which surface streamlines flow out on either side, possibly indicating a reattachment line (Green 1970). Downstream of the interaction region the flow seems to recover to the streamwise aligned flow pattern that was encountered upstream flow pattern.

Note that the shock is slightly curved and that some divergence of the oil-streaks can be observed downstream of the shock. These three-dimensional effects are attributed to the fact that the shock generator did not span the complete test section. For distances smaller than $\approx \delta$ from the centreline however the interaction appears two-dimensional. A similar spanwise uniformity within the present PIV measurement domain was demonstrated by a spanwise survey of wall-normal PIV measurements with a freestream Mach number $\mathrm{M}=2.1$ (see Humble et al. 2007).

\subsection{Perturbed interactions}

Figure 25a and $\mathrm{b}$ show oil-flow patterns of the interaction perturbed by a single row of microramps and by a staggered array of micro-ramps. The oil-flow patterns are shown in the same way as that of the undisturbed interaction in figure 24. Additionally, dashed-dotted lines are depicted to indicate the location of the micro-ramps with respect to the PIV field of view. 
Looking at the boundary layer perturbed by a single row of micro-ramps (figure 25a), it can be seen that downstream of each micro-ramp, oil accumulation initially occurs in two streamwise streaks, indicating lower wall shear stress at these locations. This is in agreement with oil-flow patterns found by Pitt Ford and Babinsky (2007) and suggests the existence of the vortex structure depicted on the right in figure 26. More downstream the oil-flow pattern disappears, indicating migration of the vortices as described in section 2.7.
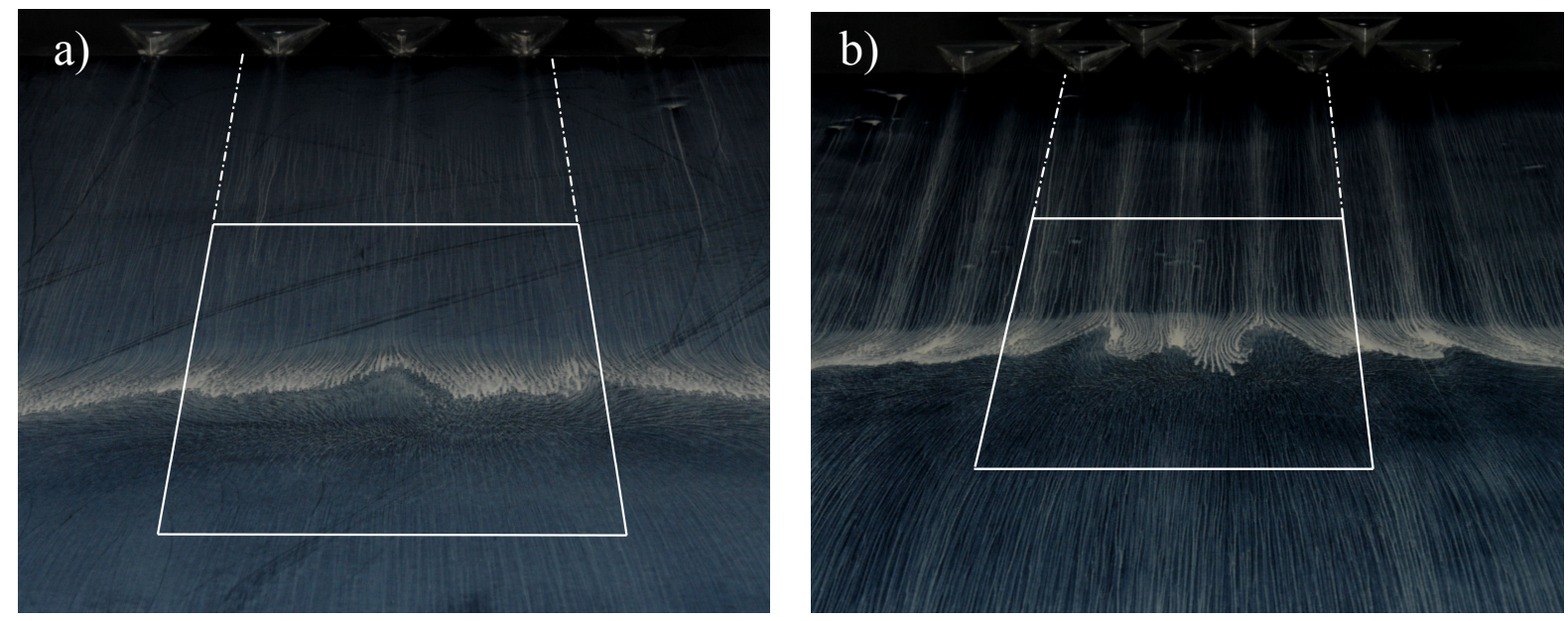

Figure 25: oil-flow visualizations of interaction perturbed by a single row of micro-ramps (left) and interaction perturbed by a staggered array of micro-ramps (right); the (projected) PIV measurement domain $(5.4 \delta \times 3.6 \delta)$ is enclosed by the solid line; dash-dot lines indicate the location of the micro-ramps w.r.t. the PIV field of view.

In figure $25 \mathrm{~b}$, the same two streamwise streaks of accumulated oil can be seen downstream of the vertices of the most downstream micro-ramps. Downstream of the most upstream microramps however only a single streak can be discerned, suggesting the vortex structure depicted on the left in figure 26. Note that in case of the staggered array the oil-flow patterns do not indicate a vortex migration away from the surface.

At the interaction region, the influence of the micro-ramps on the most upstream location of oil accumulation does not appear to be significantly large whereas their effect on the most downstream location of oil accumulation clearly is. This indicates that the micro-ramps have only a weak effect on the location of the onset of the interaction, but a much larger effect on the location of the separation line. Downstream of the vertex of a micro-ramp the separation line is located more upstream, which is consistent with (Barter and Dolling 1995; Doerffer and Swaba 2004). Note that this effect is strongest in case of the staggered array. Also observe that in that configuration the most upstream row of micro-ramps appears to have dominant influence on the organization of the flow close to the wall.

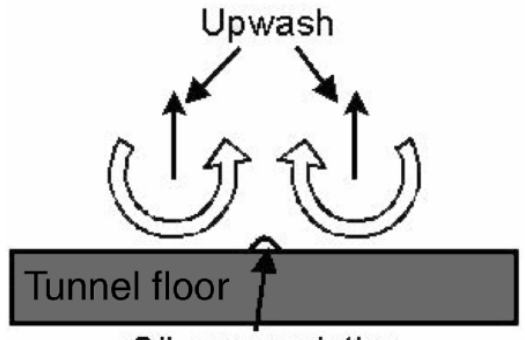

Oil accumulation

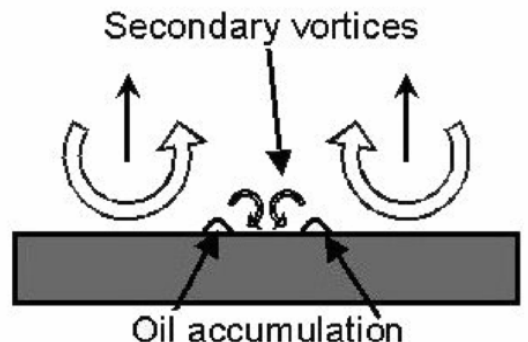

Oil accumulation

Figure 26: diagrams of two vortex structures proposed by Holden and Babinsky (2007) 


\section{Chapter 5: PIV Results and Discussion}

\subsection{Assessment of the undisturbed boundary layer}

In order to substantiate the validity of the present PIV data, it is compared with other experimental results. Figure 27 shows the mean scaled velocities found in the present investigation together with the scaled boundary layer profiles found by Tuinstra (2005) and Thomke (Fernholdz and Finley 1977). Tuinstra (2005) investigated a boundary layer by means of PIV and Thomke by using Pitot tubes. Both performed their experiments under similar conditions as those in the present investigation.

As can be seen in the figure, there is good agreement between the measured mean velocities and the other two profiles. Additionally, it can be concluded that there is not a very strong Mach dependence on the scaled velocity profile within the range considered $(1.9<\mathrm{M}<2.1)$, as was already shown by Tuinstra (2005)

It should be noted that at $y / \delta=0.1$ there is a $10 \%$ variation in the measured mean velocity throughout the field of view due to the angle of the laser sheet with respect to the wall. The point depicted in figure 27 corresponds to the mean velocity in the middle of the field of view. Other values chosen at different location agree with the depicted profiles just as well. At $y / \delta=$ 0.6 the streamwise variation in the measured mean velocity was only $1 \%$.

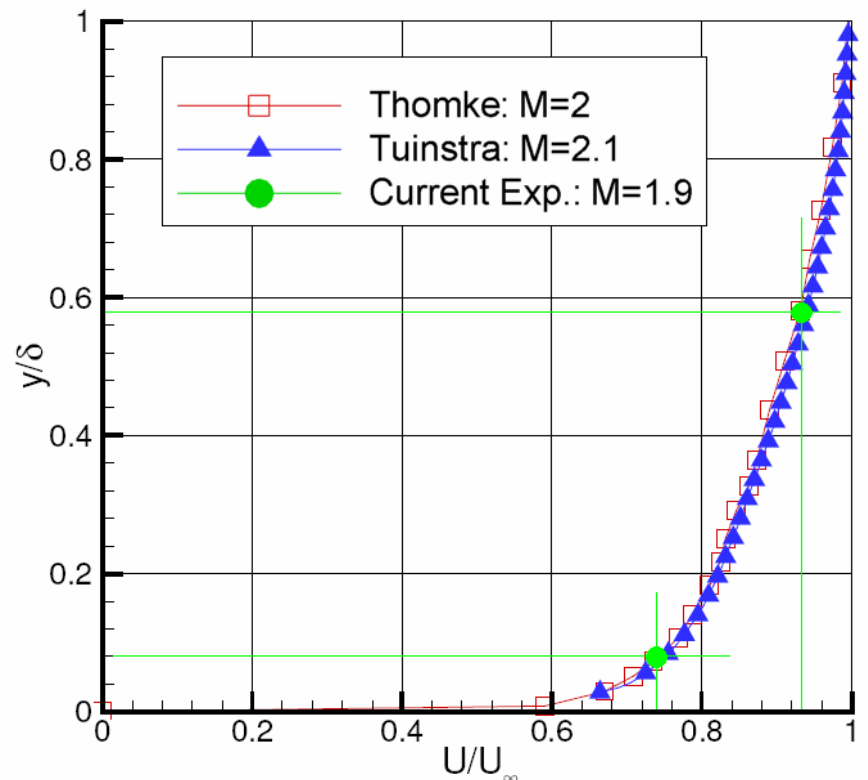

Figure 27: Scaled mean velocity profile comparisons of the current experiment with data obtained by

Thomke (Fernholdz and Finley, 1977) and Tuinstra (2005).

Figure 28 shows the RMS velocity components found in the present investigation together with tomographic PIV results of Humble et al. (2008) using, planar PIV results of Humble et al (2007) and Hou (2003) and hot-wire anemometry (HWA) and laser Doppler anemometry results of Elena and Lacharme (1988)). In order to make comparisons with the incompressible data of Klebanoff (1995) and with incompressible results of Klebanoff (1955), all data was normalized by the friction velocity $\mathrm{u}_{\tau}$ and premultiplied $\left(\rho / \rho_{\mathrm{w}}\right)^{0.5}$, where $\rho$ denotes the local 
mean density and $\rho_{\mathrm{w}}$ the density at the wall. The density ratio $\rho / \rho_{\mathrm{w}}$ was obtained by using the adiabatic Crocco-Busemann equation with the assumption of a constant recovery factor $r=$ 0.89 and a zero static pressure gradient in wall-normal direction.

There can be seen to be an agreement between the present results and the experimental results from literature. Note that the turbulence intensity in spanwise direction is close to that in the wall-normal direction, which is an expected result in moderately supersonic boundary layers (Spina et al. 1991).

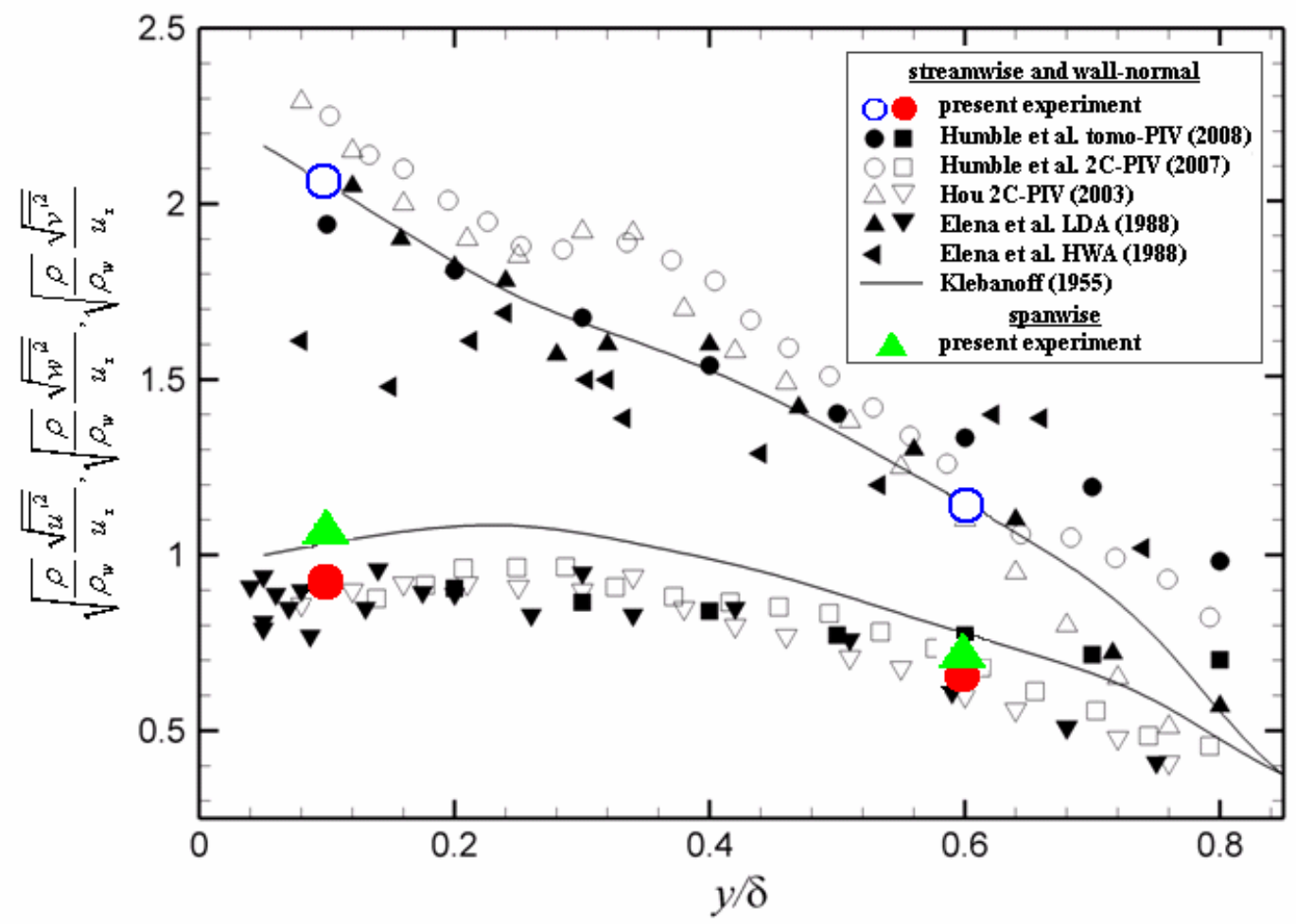

Figure 28: Streamwise, spanwise and wall-normal RMS velocity components using Morkovin's scaling; present results in streamwise and wall-normal direction are compared with experimental data of Humble et al. (2007); Humble et al. (2008); Hou (2003); Elena and Lacharme (1988) and Klebanoff (1955). 


\subsection{Mean flow organization}

In order to introduce the basic features of the interaction under investigation, contourplots of the scaled mean streamwise, spanwise and wall-normal velocities at $y / \delta=0.1$ and $y / \delta=0.6$ are depicted from top till bottom in figure 30 and figure 31 respectively. In these figures the left column corresponds to the undisturbed interaction, the middle column to the interaction perturbed by a single row of micro-ramps and the right column to the interaction perturbed by a staggered array of micro-ramps. In the plots of the mean streamwise velocity, streamlines and the sonic line are depicted as well. The sonic line was obtained by using the adiabatic Crocco-Busemann equation with the assumption of a constant recovery factor $r=0.89$ and a zero static pressure gradient in wall-normal direction:

$$
\frac{T}{T_{\infty}}=1+r \frac{(\gamma-1)}{2} M_{\infty}^{2}\left(1-\frac{U}{U_{\infty}}\right)^{2}
$$

Note that the total temperature $\left(\mathrm{T}_{0}\right)$ is known, and that therefore the freestream temperature $\left(\mathrm{T}_{\infty}\right)$ can be determined by assuming an adiabatic flow. With a known temperature distribution, the Mach distribution can now be derived by using the definition for the Mach number and the perfect gas assumption:

$M=\frac{U}{a}=\frac{U}{\sqrt{\gamma R T}}$

Where $a$ is the speed of sound and $R$ the gas constant of $287 \mathrm{~J} / \mathrm{kg} \mathrm{K}$.

In order to facilitate the reader in interpreting figure 30 and figure 31, the wall-normal organization of the interaction is depicted in figure 29 taken from Humble et al. (2007). Mean streamlines are shown superimposed with mean wall-normal velocity contours. Two horizontal solid black lines were drawn in to indicate the locations of the present measurement planes.

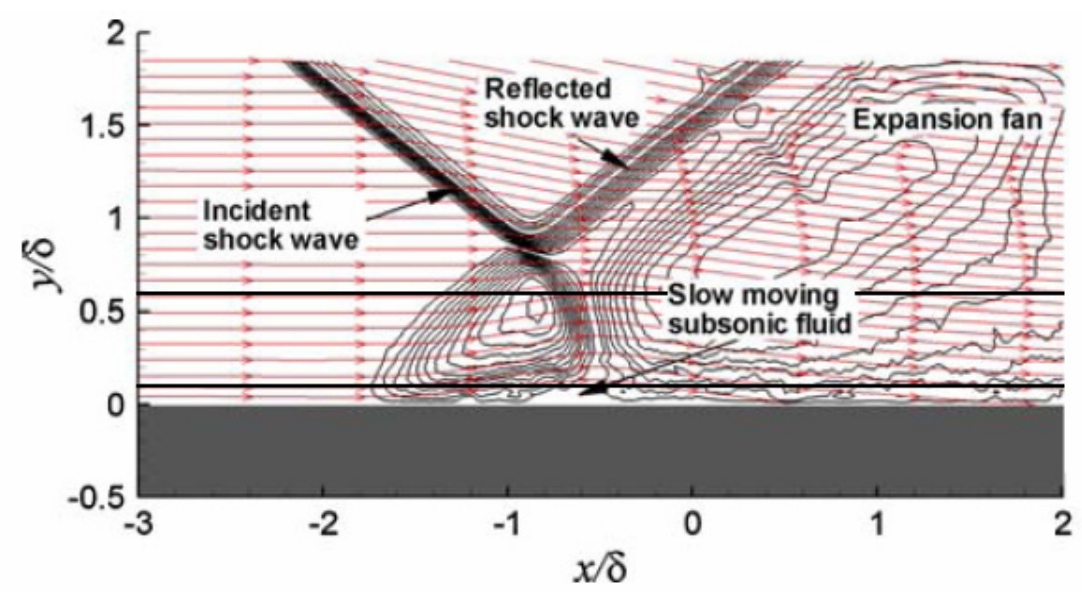

Figure 29: mean flow topology of an SWTBLI due to an $8^{\circ}$ flow deflection at Mach 2.1; mean velocity streamlines are shown along with mean wall-normal velocity distributions (adopted from Humble et al. 2007); Solid black horizontal lines show locations of the present measurement planes. 
Regarding the two-dimensionality of the undisturbed interaction, the mean velocity fields show the same two features as the oil-flow visualization: firstly, the flow appears twodimensional for distances smaller than $\approx \delta$ from the centreline of the field of view (see figure 30a). For this reason, only velocities within the streamwise band $-0.8<\mathrm{z} / \delta<0.8$ will be discussed in the text. Secondly, the streamlines diverge in spanwise direction downstream of the shock (see figure 30a and figure 31a). Note that the maximum mean velocity away from the centreline is $0.04 \mathrm{U}_{\infty}$ at $\mathrm{y} / \delta=0.1$ and half of that value at $\mathrm{y} / \delta=0.6$ (see figure $30 \mathrm{~d}$ and figure 31d).

\subsubsection{Undisturbed interaction}

Figure 30a shows that at $y / \delta=0.1$, the flow is decelerated and deflected about $2.5 \delta$ upstream of the line where the shock would impinge on the wall in absence of a boundary layer. This is consistent with observations made by Humble et al (2007). Farther downstream at $\mathrm{x} / \delta=-0.8$ the flow reaches its minimum mean streamwise velocity of about $0.16 \mathrm{U}_{\infty}$, which is approximately $20 \%$ of its value in the incoming boundary layer. Figure $30 \mathrm{~g}$ shows that the mean wall-normal velocity is not fully converged at this streamwise location. Nevertheless somewhere between $\mathrm{x} / \delta=-1.4$ and $\mathrm{x} / \delta=-0.8$, the flow can be seen to become parallel to the wall again. Downstream of $\mathrm{x} / \delta=-0.8$, the flow is moving back to the wall while it accelerates. At $\mathrm{x} / \delta=1.0$ the boundary layer has recovered to $0.45 \mathrm{U}_{\infty}$ which is about $60 \%$ of its value upstream of the interaction. It is remarked that on the mean the interaction does not lead to reversed flow at $\mathrm{y} / \delta=0.1$.

Farther away from the wall, at $y / \delta=0.6$, (see left column of figure 31 ) the start of the deceleration region is located about $0.5 \delta$ more downstream than at $\mathrm{y} / \delta=0.1$, which is consistent with observations made by Humble et al (2007). In the first part of the interaction the flow is deflected away from the wall and decelerated from $0.94 \mathrm{U}_{\infty}$ to $0.74 \mathrm{U}_{\infty}$. After this first deflection, the flow is deflected back parallel to the wall and further decelerated to $0.60 \mathrm{U}_{\infty}$. The existence of two distinct deceleration regions is consistent with Humble et al. (2007), who showed that at $y / \delta=0.6$ the flow first converging compression waves that coalesce to form the reflected shock (see figure 29). More downstream, the flow encounters the incident shock wave and secondary waves that are formed due to its refraction in the boundary layers.

It can be seen from figure $31 \mathrm{a}$ that the deceleration region that is associated with the incident shock causes a more widespread deflection and deceleration of the flow than the reflected compression. Humble et al. (2007) showed that this can be attributed to the larger streamwise excursions of the latter. Due to the averaging effect, these streamwise excursions lengthen the compression region in the mean velocity field. In section 5.3 and 5.5 the motion of the reflected shock found in the present investigation will be discussed in more detail.

At $\mathrm{x} / \delta=1.0$ the boundary layer has recovered to $0.80 \mathrm{U}_{\infty}$ which is $85 \%$ of the velocity upstream of the interaction. Note that the recovery in terms of this percentage is $15 \%$ higher at $\mathrm{y} / \delta=0.6$ than at $\mathrm{y} / \delta=0.1$. Observe that both at $\mathrm{y} / \delta=0.1$ and at $\mathrm{y} / \delta=0.6$ the acceleration of the flow is a more gradual process than the deceleration, which is consistent with literature (Délery and Marvin 1986). 
$\begin{array}{llllll}\infty & 0 & n & 0 & 0 & 0 \\ 0 & 0 & 0 & 0 & 0 & 0 \\ 0 & 0\end{array}$
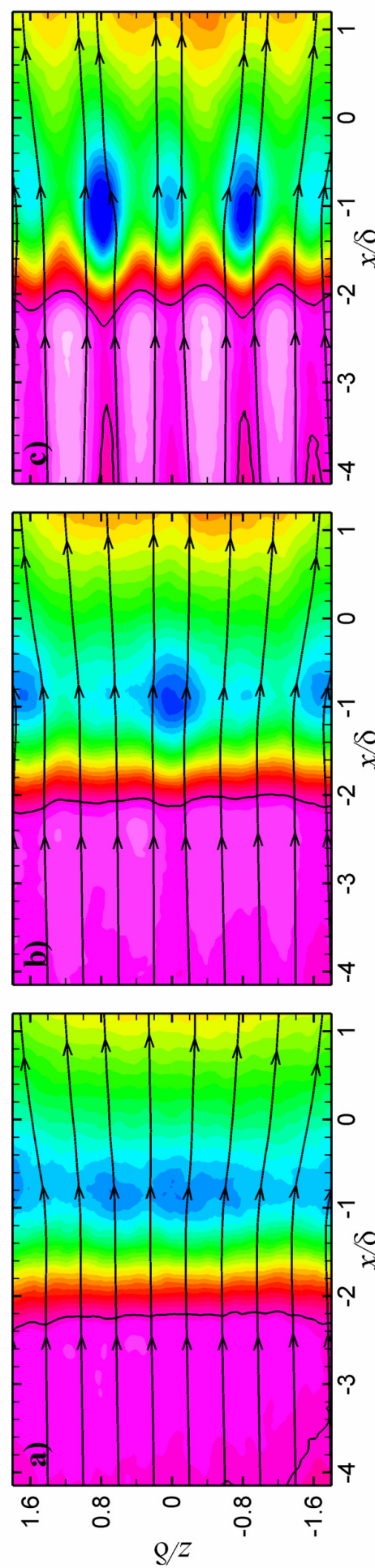
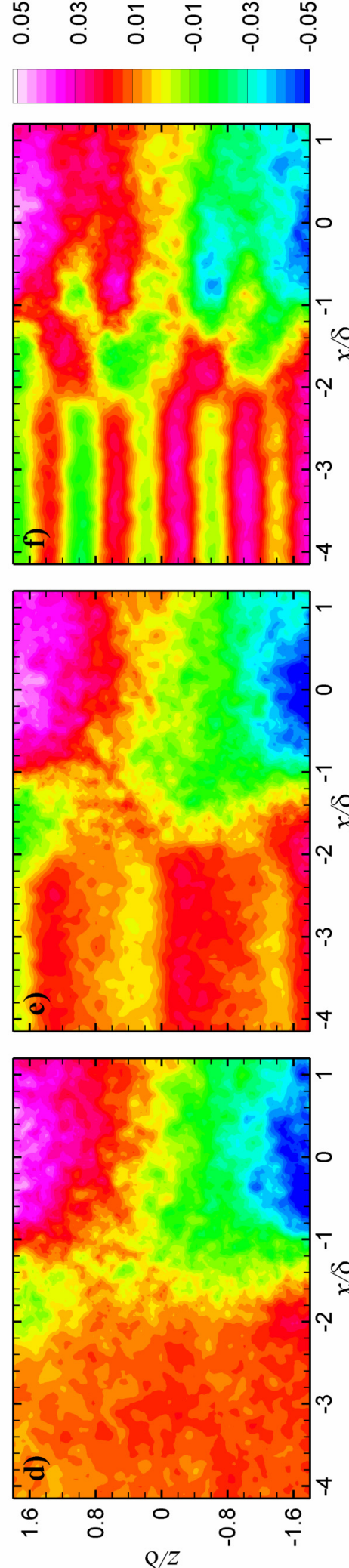

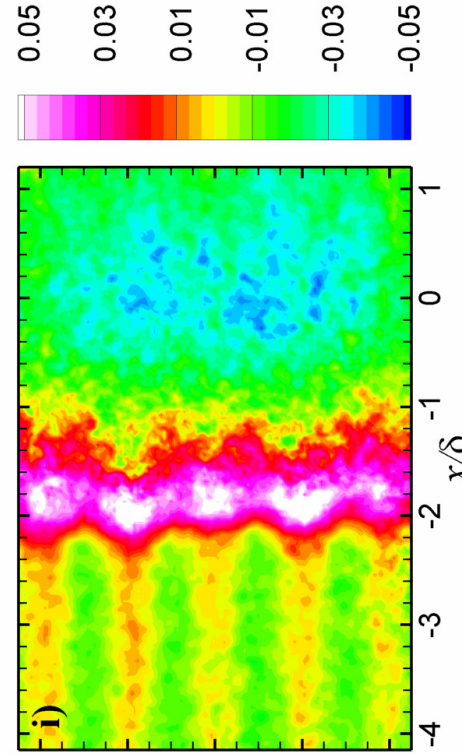

.

के

름

范

氞

है응

일

. ส อั้ 플 巳 E क 昰
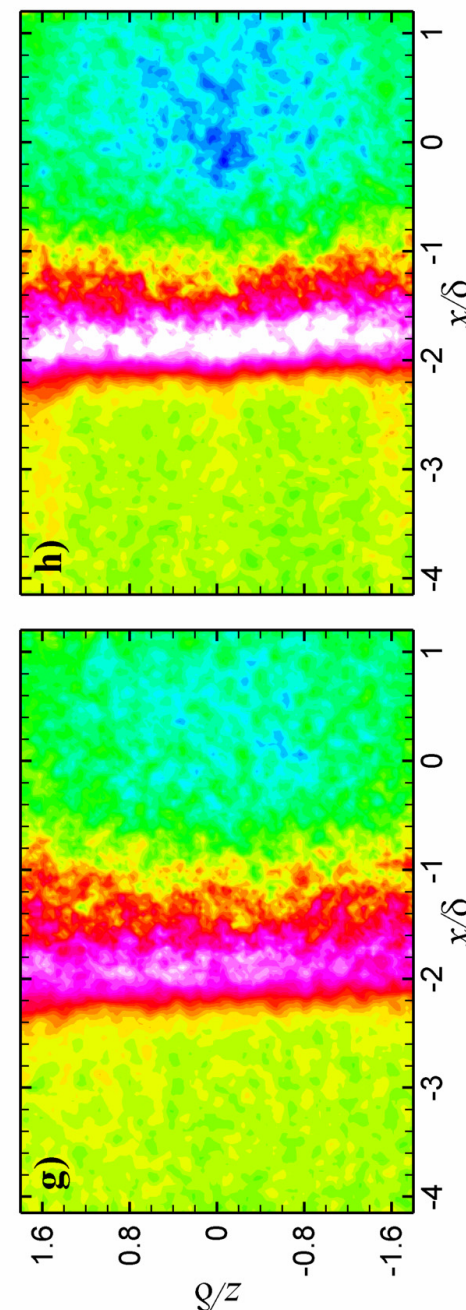

ஊ

용

을 쁠

$\unrhd^{8} . \equiv$

-

를

ㄹ

을 을

总

8 .

$\checkmark$ 约

i

気

를

$\underset{3}{0}$

२

$=$ 苟

$\because$ a

충

此

党

응

을

을

을

ह

ฮั

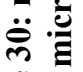

능

至 


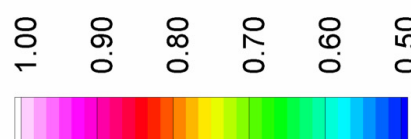
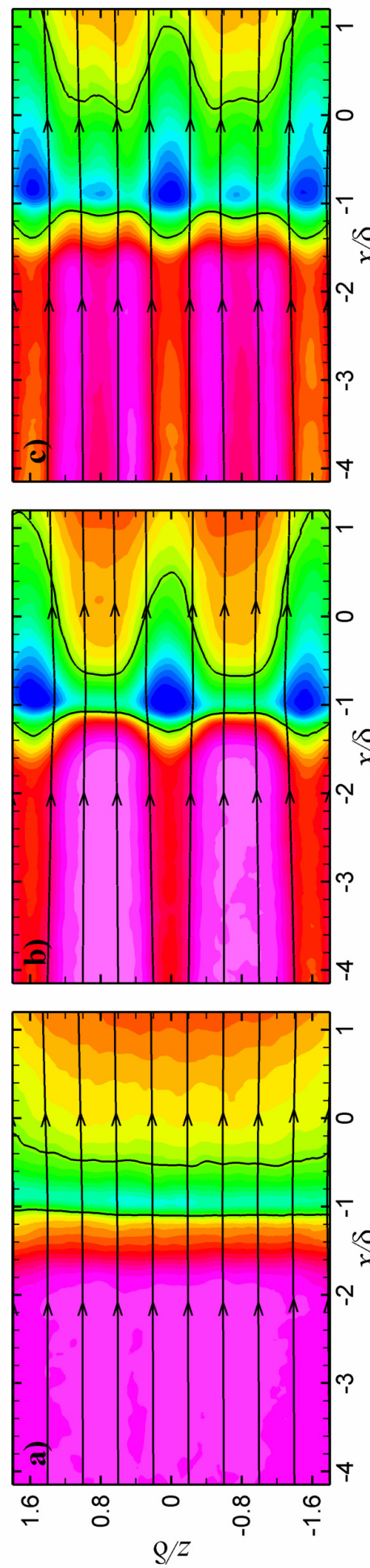
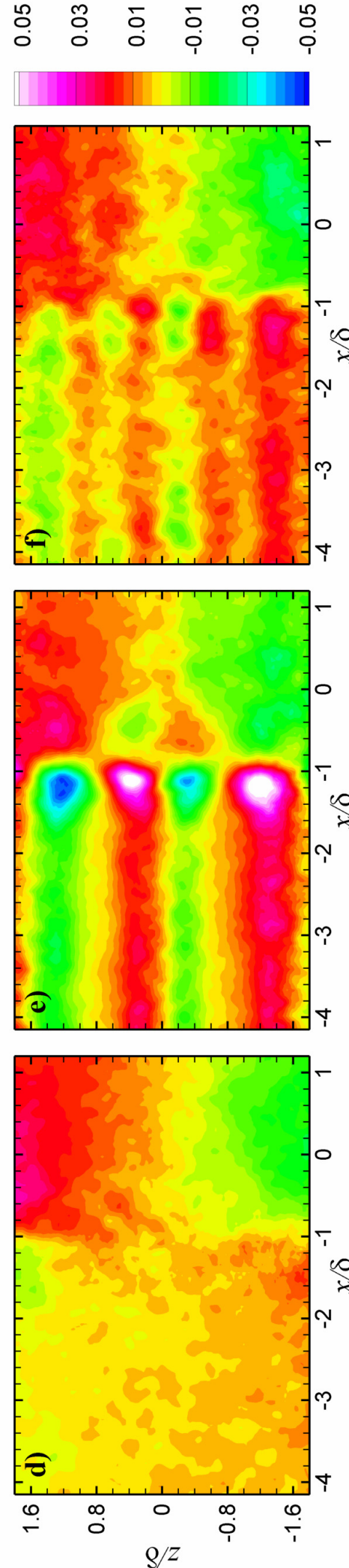

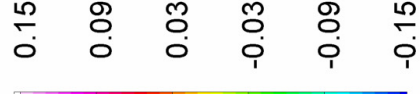

일
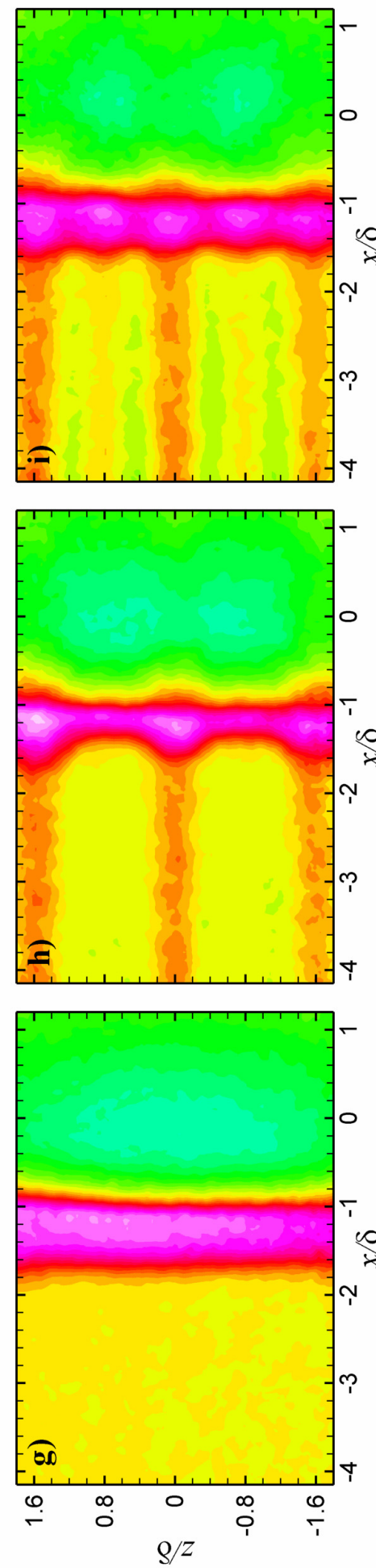

产

要政

$\Xi$

$\sum^{8}$

$=$

$\ddot{\circ}$

11

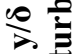

需

등

을 을

을

。

일

छ

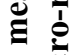

ले

능

赵 


\subsubsection{Perturbed interactions}

In order to assess the influence of the micro-ramps on the mean flow throughout the interaction, the mean velocity fields will be discussed (see figure $30 \mathrm{~b}$ and figure 31 ). Remember from section 3.2.3 that in the single row configuration, micro-ramps apices are located at $\mathrm{z} / \delta=-1.6,0$ and 1.6 and in the staggered configuration at $\mathrm{z} / \delta=-1.6,-0.8,0,0.8$ and 1.6. It is remarked that influence of both configurations on a boundary layer without an impinging shock does not significantly change in streamwise direction within the field of view.

Figure $30 \mathrm{~b}, \mathrm{c}$ and figure $31 \mathrm{~b}, \mathrm{c}$ show that the flow is no longer two-dimensional. Instead, there is a periodic spanwise variation in mean streamwise velocity throughout the field of view. The lowest velocity occurs downstream of the vertices of the ramps, which is consistent with results found at freestream Mach numbers ranging from 1.5 to 5 (Barter and Dolling 1995; Holden and Babinsky 2007). Conversely, Pitt Ford and Babinsky (2007) reported that close to the wall at $\mathrm{y} / \delta=0.1$, the mean velocity is highest downstream of a vertex.

Concerning the spanwise organization of streamwise velocity induced by the micro-ramps three major observations can be made: firstly, the micro-ramps do not appear to have a strong influence on the location of the onset of the interaction, which is associated with the most upstream location of flow deceleration. Note that this is consistent with oil-flow results. Secondly comparing figure $30 \mathrm{~b}$ and figure $30 \mathrm{c}$ it becomes clear that at $\mathrm{y} / \delta=0.1$ the spanwise variation in mean streamwise velocity is much larger downstream of the staggered array than downstream of the single row of micro-ramps. Thirdly, inspecting figure 30c and figure 31c it seems that downstream of the staggered the mean flow organization at $y / \delta=0.1$ is dominated by the most upstream row of micro-ramps at $\mathrm{z} / \delta= \pm 0.8$, again consistent with oil-flow results, while at $\mathrm{y} / \delta=0.6$ it is dominated by the downstream row of micro-ramps at $z / \delta=-1.6,0$ and 1.6.

In order to determine the spanwise averaged effects of both configurations of micro-ramps, the mean streamwise velocity averaged between $-0.8<\mathrm{z} / \delta<0.8$ at $\mathrm{y} / \delta=0.1$ and at $\mathrm{y} / \delta=0.6$ are depicted on the left and right in figure 32 respectively.
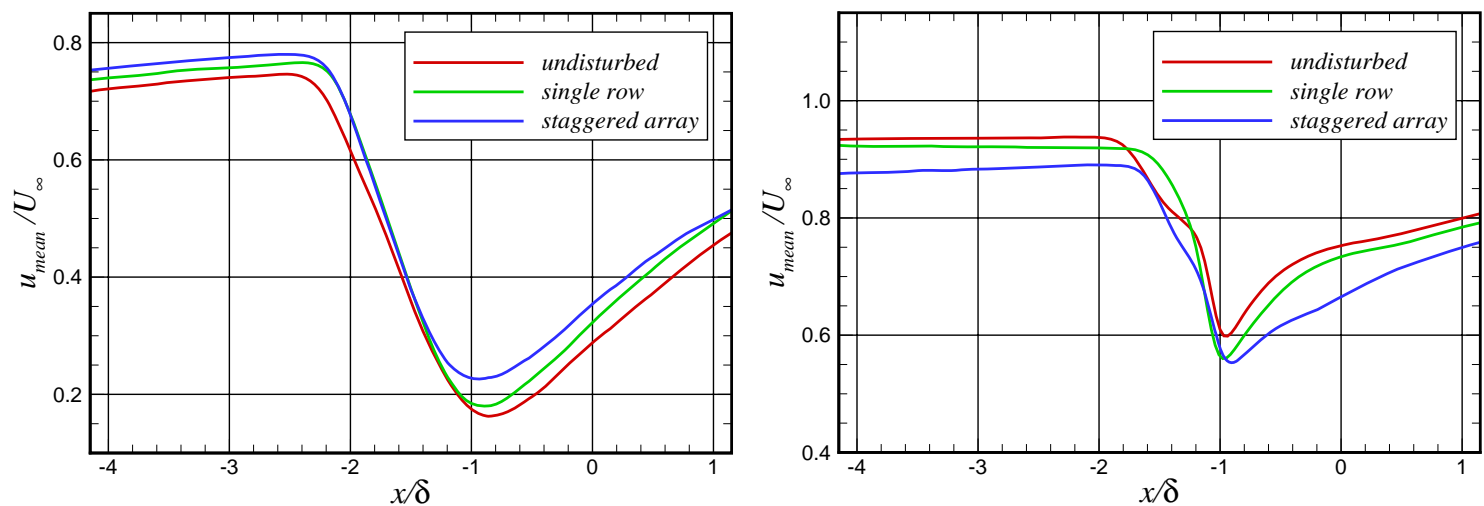

Figure 32: mean streamwise velocity spanwise averaged over $-0.8<z / \delta<0.8 ;$ at $y / \delta=0.1$ (left) and at $y / \delta=$ 0.6 (right)

In general the effect of the micro-ramps on the spanwise averaged mean velocity, although larger than the local uncertainty, appears to be weak. The micro-ramps seem to cause an overall increase in mean streamwise velocity with respect to the undisturbed interaction at $y / \delta$ $=0.1$. At $y / \delta=0.6$, their effect is opposite as they decrease the mean streamwise velocity with 
respect to the undisturbed interaction. This observation is consistent with the flow organization in which the micro-ramps generate streamwise vortices that mix low-velocity fluid from the lower part of the boundary layer into the upper part of the boundary layer and vice versa as proposed by Holden and Babinsky (2007) and Pitt Ford and Babinsky (2007). The effect of the staggered array can be seen to be larger than that of the single row at both $\mathrm{y} / \delta=0.1$ and at $\mathrm{y} / \delta=0.6$, suggesting that the former configuration induces a stringer mixing in the boundary layer region between the two measurement planes.

Regarding the mean wall-normal velocity, figure $30 \mathrm{~h}$ shows that the single row of microramps induces no significant spanwise organization of this quantity at $y / \delta=0.1$. Farther away from the wall at $\mathrm{y} / \delta=0.6$, however, the deflection away from the wall does show a spanwise organization (see figure 31h): like in the incoming boundary layer the wall-normal mean velocity is higher downstream of the vertices of the micro-ramp. The same behaviour can be observed downstream of the staggered array, at both $\mathrm{y} / \delta=0.1$ and $\mathrm{y} / \delta=0.6$ (figure $30 \mathrm{i}$ and figure 31i).

In the centre of the field in figure $31 \mathrm{e}$ a very distinct pattern in the mean spanwise velocity field can be seen, indicating the following behaviour of the mean flow in the interaction region downstream of the micro-ramps: in the first part of the interaction region $(-1.6<\mathrm{x} / \delta<-$ $0.8)$ the flow moves away from the centreline and more downstream $(-0.8<\mathrm{x} / \delta<0.0)$ it moves back again. Figure 30f shows that also downstream of the staggered array the streamlines first diverge in the first part of the interaction and subsequently converge again. Note that the organization of the mean spanwise velocity is dominated by the most upstream row of microramps $(\mathrm{z} / \delta= \pm 0.8)$, which is consistent with previous observations of the mean streamwise velocity organization.

This organization of the mean spanwise velocity is believed to be the spanwise equivalent of the wall-normal flow deflection in a two-dimensional interaction. In the latter, the flow is moving away from the wall in the first part of the interaction and back to the wall in the second part. In the considered perturbed three-dimensional interactions the flow is also deflected to the sides downstream of the micro-ramps, hence the diverging streamlines. More downstream where the subsonic region becomes smaller, the flow moves back to the wall and also back in spanwise sense, causing the streamlines to move closer together again.

\subsubsection{Vorticity distribution}

In order to make statements regarding the vortex organization of the interaction, the out-ofplane vorticity distributions at $\mathrm{y} / \delta=0.1$ and $\mathrm{y} / \delta=0.6$ are shown in figure 33 and figure 34 respectively. The out-of-plane mean vorticity was determined using:

$$
\bar{\omega}_{y}=\frac{\delta}{U_{\infty}}\left(\frac{\partial \bar{v}}{\partial x}-\frac{\partial \bar{u}}{\partial z}\right)
$$

where central-differencing was used to compute the gradients. The depicted results will only be discussed qualitatively.

Figure 33a and figure 34a show that streamwise elongated regions of increased vorticity are apparent in the undisturbed interaction region. Since the average out-of-plane vorticity component should be homogeneous at a given height within the boundary layer, the presence 
of these regions it attributed to a lack of statistical convergence. Their presence is consistent with PIV results from Ganapathisubramani et al. (2007), who showed that the instantaneously vorticity downstream of the shock in compression ramp interaction was 2-3 times higher than in the incoming boundary layer.

Figure $33 \mathrm{~b}$, c and figure $34 \mathrm{~b}$, c show that in the incoming boundary layer, the micro-ramps create pairs of streamwise streaks of mean vorticity with the same sign and magnitude. In both configurations the streaks span about $0.4 \delta$. In case of the single row of micro-ramps this is half the pitch of the micro-ramps, while in case of the staggered array it is the full pitch. Note that at $y / \delta=0.1$ the absolute mean vorticity levels in the incoming boundary layer perturbed by the single array are 3 to 4 times higher than in the boundary layer perturbed by the single row (see figure 33). This observed larger influence of staggered array of microramps at $y / \delta=0.1$ compared to that of the single row configuration is consistent with previous observations. At $y / \delta=0.6$ the absolute mean vorticity levels for both configurations are about the same (figure 34).

The figures show that the magnitude of the mean vorticity typically starts to increase at the onset of interaction. As mentioned earlier this amplification was also found from instantaneous results by Ganapathisubramani et al. (2007). In case of the single row, the absolute mean vorticity increases by approximately a factor 2 with respect to the incoming boundary layer and in case of the staggered array by a factor 3 to 4 , leading to a maximum absolute mean vorticity of 0.4 at about $\mathrm{x} / \delta=-1.1$ in case of the single row and of 1.4 at about $\mathrm{x} / \delta=-1.3$ in case of the staggered array. More downstream the mean vorticity levels become lower again, while the streaks become spatially more distorted. At $y / \delta=0.6$ a factor 2 amplification can be seen in case of the single row, but no amplification can be discerned for the staggered configuration.

Figure $34 \mathrm{c}$ shows that at $\mathrm{y} / \delta=0.6$, weak streaks at $\mathrm{z} / \delta= \pm 0.8$, induced by the most upstream row of micro-ramps in the staggered set, seem to be embedded within the original rows (the single row configuration), while at $y / \delta=0.1$ the effect of the most upstream row is dominant (see figure 33c). 
웅 운
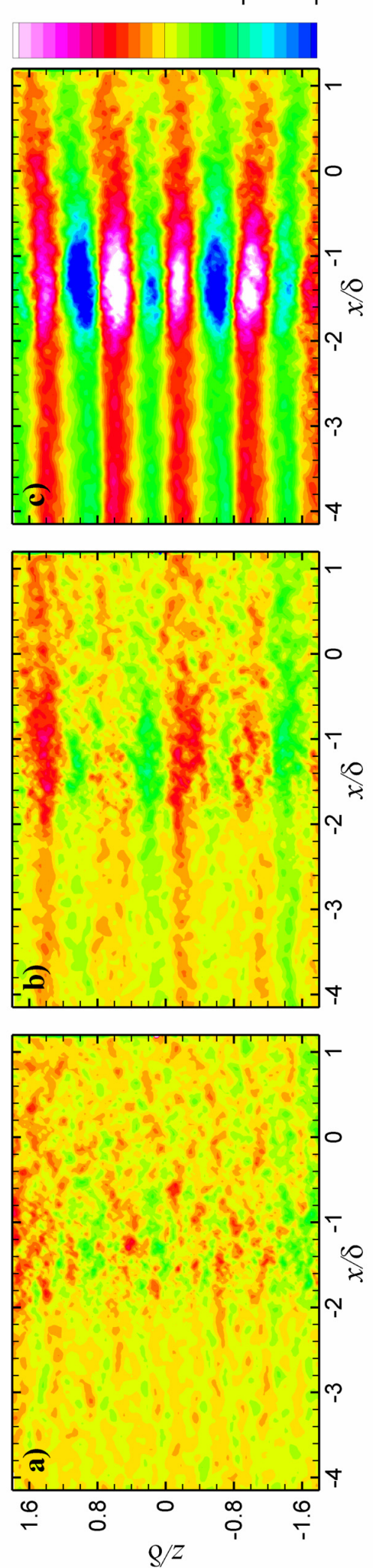

$\begin{array}{lllll}\circ & 0 & 0 & 0 & 0 \\ \circ & 0 & 0 & 0\end{array}$
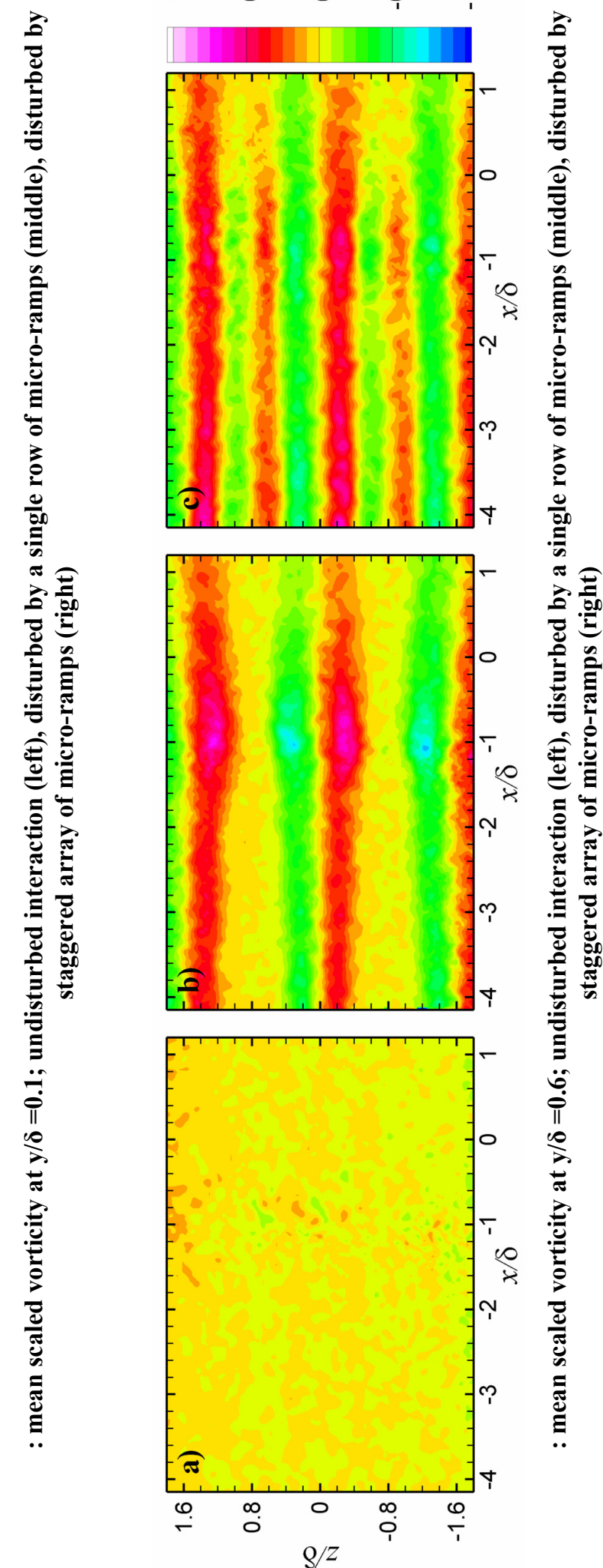

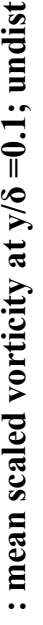




\subsubsection{Structure of the perturbed boundary layers}

In order to determine the influence of the micro-ramps on the interaction it is useful to look into the mean structure of the incoming boundary layer. In figure 35 the mean scaled streamwise velocity upstream of the interaction (averaged over $-4.0<\mathrm{x} / \delta<-2.5$ ) is plotted. The mean streamwise velocity at $y / \delta=0.6$ is shown by the upper lines and that at $y / \delta=0.1$ by the lower lines.

As was seen earlier local minima in the mean streamwise velocity are located downstream of the apices of the micro-ramps, The lower green line in figure 35 shows that two additional local minima are present in between two micro-ramps $(\mathrm{z} / \delta= \pm 0.8)$. The presence of these minima suggests a small effect of the micro-ramps on the flow at these locations as was also seen in the vorticity plots.

Inspecting figure 35, the following differences between the two perturbed interactions can be found: firstly, downstream of the staggered configuration at $y / \delta=0.6$ the mean streamwise velocity is always lower than in the undisturbed boundary layer (see upper blue line), whereas downstream of the single row of micro-ramps the streamwise velocity is locally higher than in the undisturbed boundary layer (see upper green line). Secondly, it is noted that at $y / \delta=0.6$ the staggered configuration causes larger velocity deficits downstream of the micro-ramps at $\mathrm{z} / \delta=-1.6,0$ and 1.6 than in case of the single row (compare upper blue and green line). Thirdly, at $\mathrm{y} / \delta=0.6$ the staggered array induces two relatively weak minima in the mean streamwise velocity at $\mathrm{z} / \delta= \pm 0.8$ (see upper blue line). And finally, as mentioned earlier the effect of the staggered array of micro-ramps on the flow at $y / \delta=0.1$ is much larger than that in case of the single row configuration (see lower blue line. As a result, the mean streamwise velocity at $\mathrm{z} / \delta=-0.8$ and 0.8 is lower than in the unperturbed flow. Again it is observed that the most downstream row of micro-ramps in the staggered set is dominant at $y / \delta=0.6$, while at $y / \delta=0.1$ the effect of the most upstream row is largest.

Figure 36 shows the mean streamwise velocity downstream of the interaction at $\mathrm{x} / \delta=1.0$. Just like in figure 35 , the mean streamwise velocity at $y / \delta=0.6$ is shown by the upper lines and that at $\mathrm{y} / \delta=0.1$ by the lower lines. As was seen before, the micro-ramps have a negative effect on the recovery at $y / \delta=0.6$ and a positive effect at $y / \delta=0.1$. Comparing figure 35 and figure 36 it can be seen that this can be attributed to the fact that the mean streamwise velocity qualitatively seem to recover its organization upstream of the interaction. There are however some differences with respect to this upstream organization: in general, the difference between the local minima and maxima has diminished, in fact the velocity distribution seems to be diffused. As a result, at $y / \delta=0.6$ (see upper lines) there are no longer regions in which the mean streamwise velocity is higher than in the unperturbed interaction and at $y / \delta=0.1$ (lower lines) there are no longer regions where the mean velocity is lower. Also note that, at $\mathrm{y} / \delta=0.6$ the effect of the most upstream row of micro-ramps in the staggered, located at $\mathrm{z} / \delta=$ -0.8 and 0.8 , can no longer clearly be discerned (see upper blue line). 


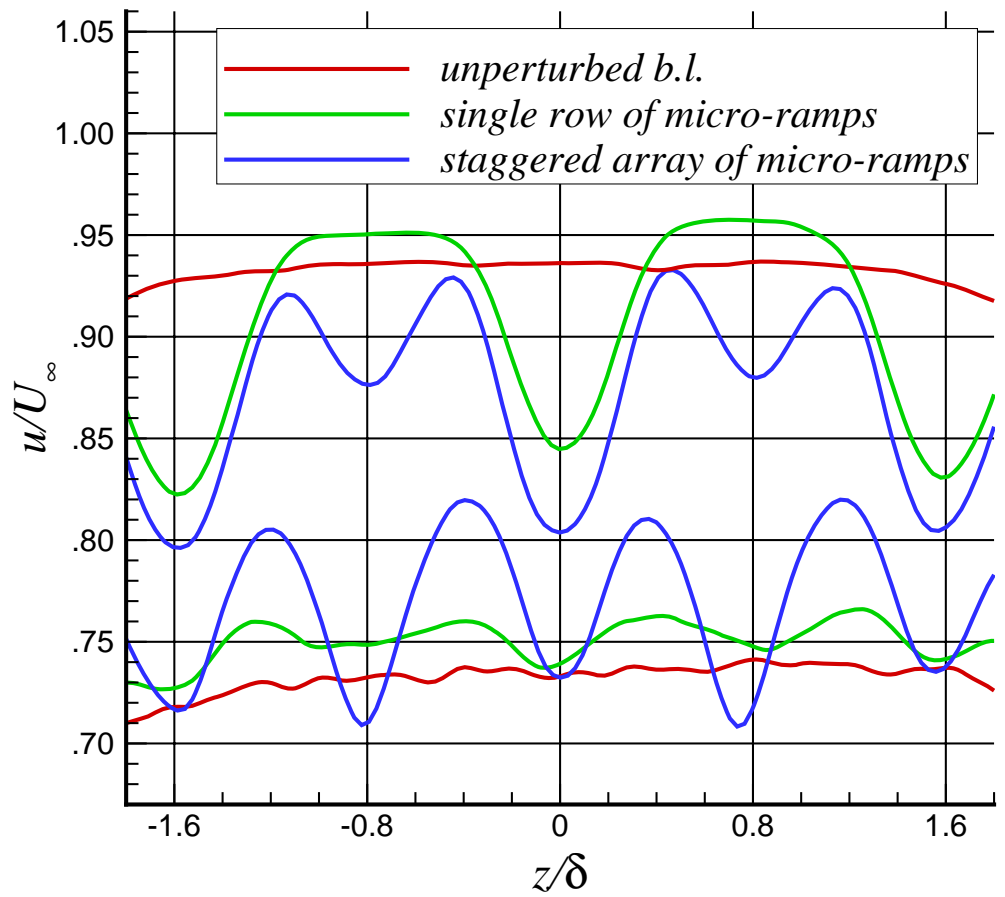

Figure 35: mean streamwise velocity upstream of the interaction; averaged over over $-4.0<x / \delta<-2.5$; at $\mathrm{y} / \boldsymbol{\delta}=\mathbf{0 . 6}$ (upper lines) and $\mathbf{y} / \boldsymbol{\delta}=0.1$ (lower lines)

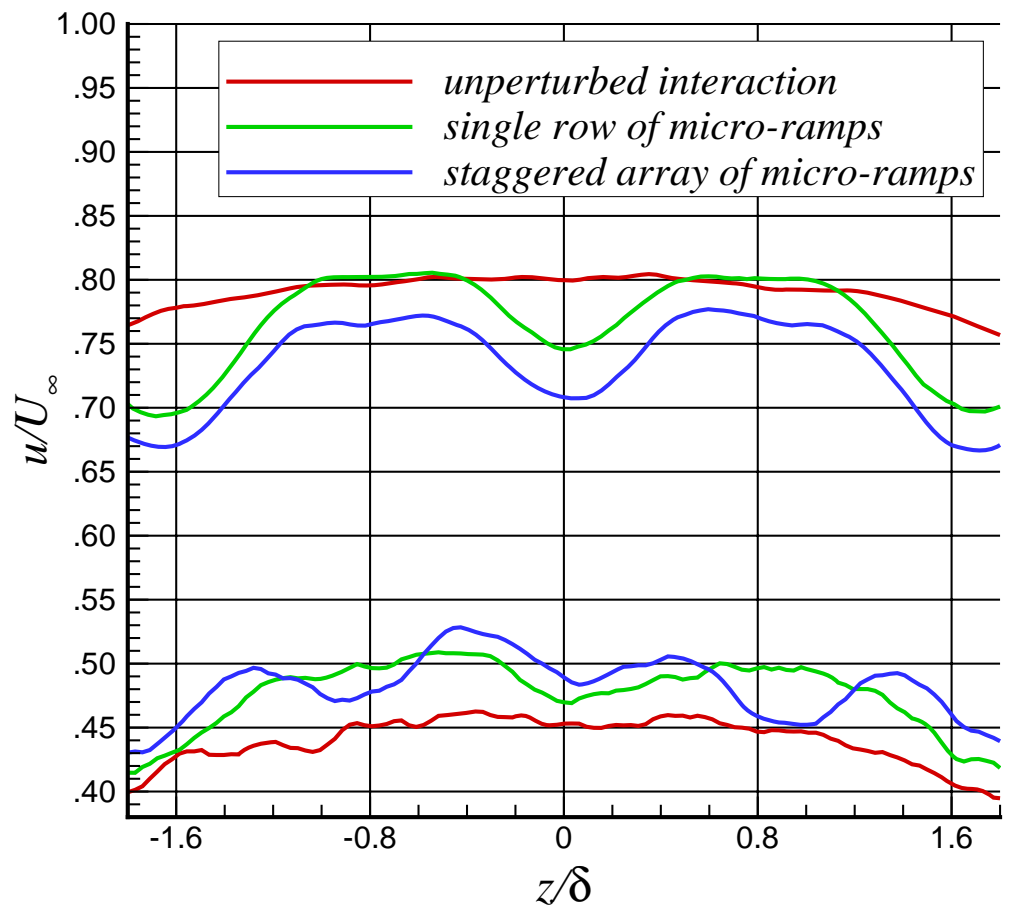

Figure 36: mean streamwise velocity downstream of the interaction; at $x / \delta=1.0 ; y / \delta=0.6$ (upper lines) and $\mathbf{y} / \delta=0.1$ (lower lines) 
In the foregoing evidence was found for mixing between the lower and higher part of the boundary layer. In order to investigated this idea more closely, a wall-normal cross-section at $\mathrm{x} / \delta=-3.0$ is depicted in figure 37. The streamwise velocity distribution was obtained by means of linear interpolation of the measured data at $y / \delta=0.1$ and $y / \delta=0.6$. The author is aware that linear interpolation is not a very accurate way to predict the flow in the boundary layer. It is therefore emphasised that the obtained result is shown only for illustration purposes.

The figure puts in evidence for pairs of counter-rotating streamwise vortices in the boundary layer, that induce a pronounced mixing by moving low-velocity fluid away from the wall downstream of the micro-ramp's vertices $($ e.g. $\mathrm{z} / \delta=0)$ and high- velocity fluid towards the wall at intermediate spanwise positions. The semi-transparent arrows in this figure are meant to illustrate the vortices but do not correspond to their size, which cannot be accurately be determined with the data at hand.

For comparison the flow organization in a wall-normal cross-section downstream of the interaction at $\mathrm{x} / \delta=-1.0$ is plotted in figure 36. Like in figure 37 , the mean streamwise velocity distribution was obtained by means of linear interpolated from the measured data at $\mathrm{y} / \delta=0.1$ and $\mathrm{y} / \delta=0.6$. It should be noted that compared to figure 37 the depicted range of velocities is larger and the vectors are shown three times as small.

The vectors show that the mean flow is moving towards the wall quite uniformly. Especially at $\mathrm{y} / \delta=0.1$, a velocity component away from the centreline $(\mathrm{z} / \delta=0)$ can be seen, as was already reported in previous sections. The interpolated mean streamwise velocity contours shows a weak spanwise variation that could be interpreted as an indication for the existence of streamwise vortices. It may also very well be that the spanwise variation in mean streamwise velocity downstream of the interaction are not induced by local streamwise vortices, but is simply a residue of the variations upstream of the interaction. This latter suggestion is supported by the observation that the organization of the mean wall-normal vorticity becomes distorted downstream of the interaction.

Observe figure 36 that the mean flow between two vertex locations is induced downwards. It is therefore conceivable that the most downstream row of micro-ramps creates a flowfield that moves the flow coming from the most upstream ramps closer to the wall. This provides a physical explanation for observation that in case of the staggered configuration the most downstream row of micro-ramps has a dominant influence on the mean organization at $y / \delta=$ 0.6 , while at $\mathrm{y} / \delta=0.1$ the effect of the most upstream row is largest.

Remember that it was found the effect of the staggered array of micro-ramps on the mean flow at $\mathrm{y} / \delta=0.1$ is much greater than that in case of the single row configuration. The existence of streamwise vortices, as hinted by figure 36, provides a framework to speculate on the reason for this difference. In the previous section that streamwise streaks of mean wallnormal vorticity with the same sign and magnitude exist downstream of the micro-ramps and that for both configurations these streaks span about $0.4 \delta$. If now it is assumed that the width of these streaks can serve as an indication for the characteristic widths of the streamwise vortices, it seems that in case of the staggered array, the pitch of the streamwise vortices more or less equals the characteristic widths of the streamwise vortices. As a consequence, the downward velocities induced by the vortices may amplify each other, thereby increasing the effect of the staggered array close to the wall. 


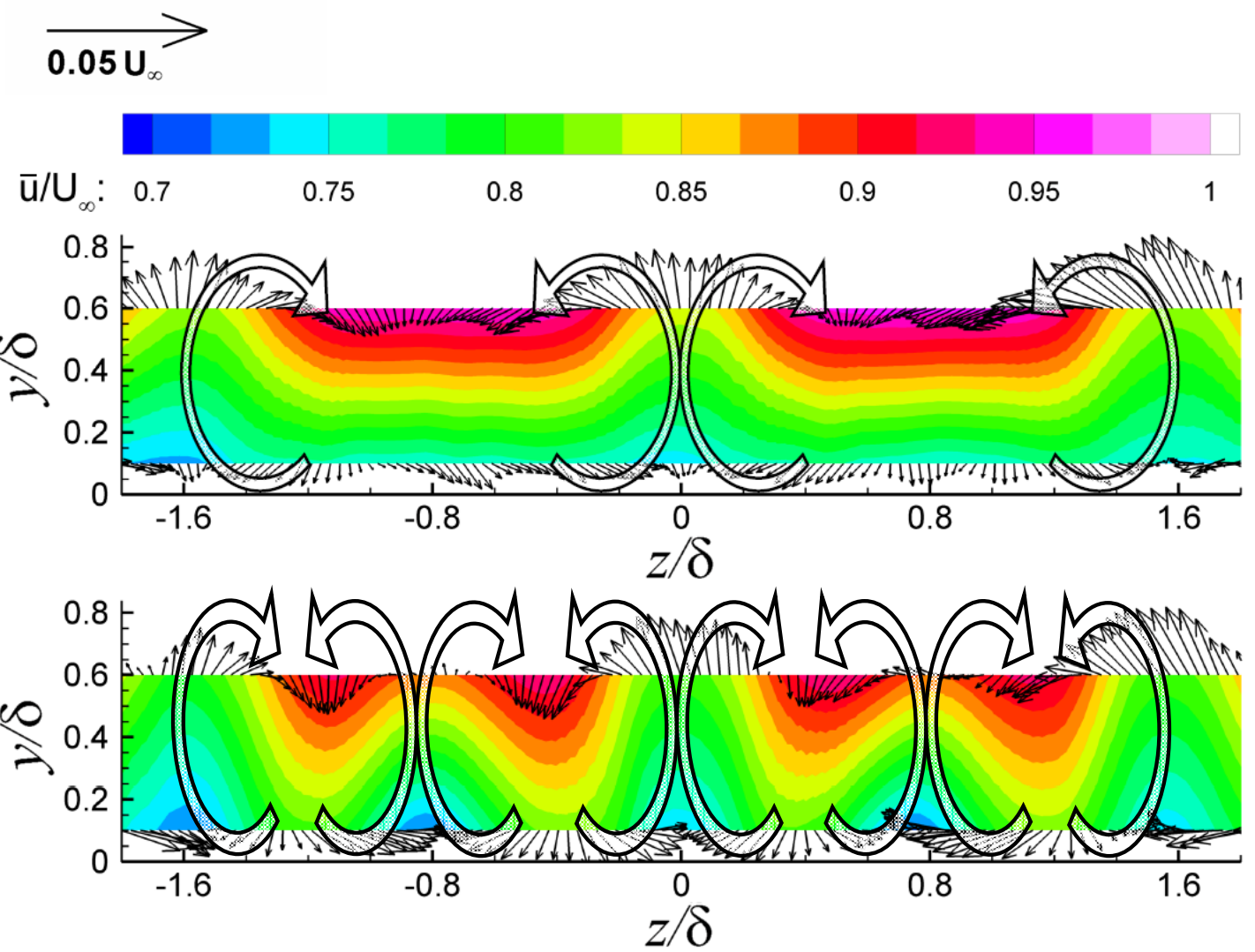

Figure 37: wall-normal cross-section at $\mathbf{x} / \boldsymbol{\delta}=\mathbf{- 3 . 0}$; vectors of measured data are shown together with interpolated mean streamwise velocity of the boundary layer perturbed by a single row of micro-ramps (upper) and by staggered array of micro-ramps (lower); size of drawn in semi-transparent arrows was chosen arbitrarily
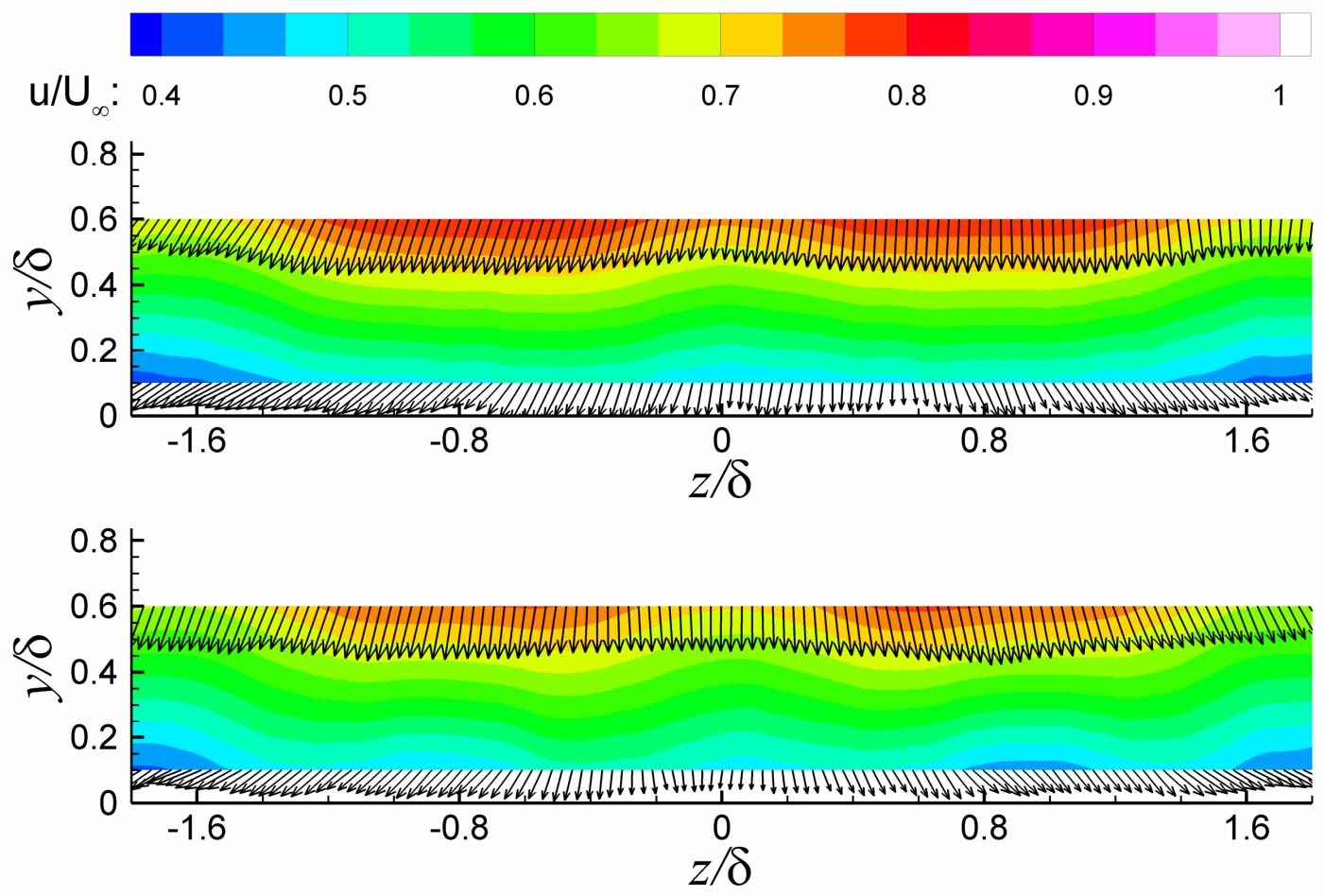

Figure 38: wall-normal cross-section at $x / \delta=1.0$; vectors of measured data are shown together with interpolated mean streamwise velocity of the boundary layer perturbed by a single row of micro-ramps (upper) and by staggered array of micro-ramps (lower); vectors are depicted three times as small as in 
Another explanation for the greater influence of the staggered array on the mean flow close to the wall with respect to that of the single row configuration was provided by the oil-flow patterns, which hinted vortex migration away from the surface downstream of the single row of micro-ramps. Remember from section 2.7 that this migration was studied by Pearcey (1961), who regards it as the primary cause for the limited range of effectiveness of vortex generators. According to Pearcey (1961), it should therefore be a design object to postpone the migration of the vortices away from the surface as long as possible. In this sense it appears that the staggered array better conforms to these design rules.

Following Pearcey (1961), two vortices with a common flow up induce each other away from the surface while moving together whereas two vortices with a common flow down induce each other towards the surface while moving apart. As a consequence the vortex migration is dependent on the ratio $\mathrm{D} / \mathrm{d}$, where $\mathrm{D}$ denotes the constant cell span and $\mathrm{d}$ the variable distance between two neighbouring vortices chosen so that in the region between them the induced flow is towards the surface (see figure 39). Pearcey (1961) found that when $\mathrm{D} / \mathrm{d}<2$, the vortices immediately start to move away from the surface, but that when $\mathrm{D} / \mathrm{d}>2$, the vortices first move towards the surface while grouping together in pairs, before they lift of the surface thereby postponing this final event (see figure 39).

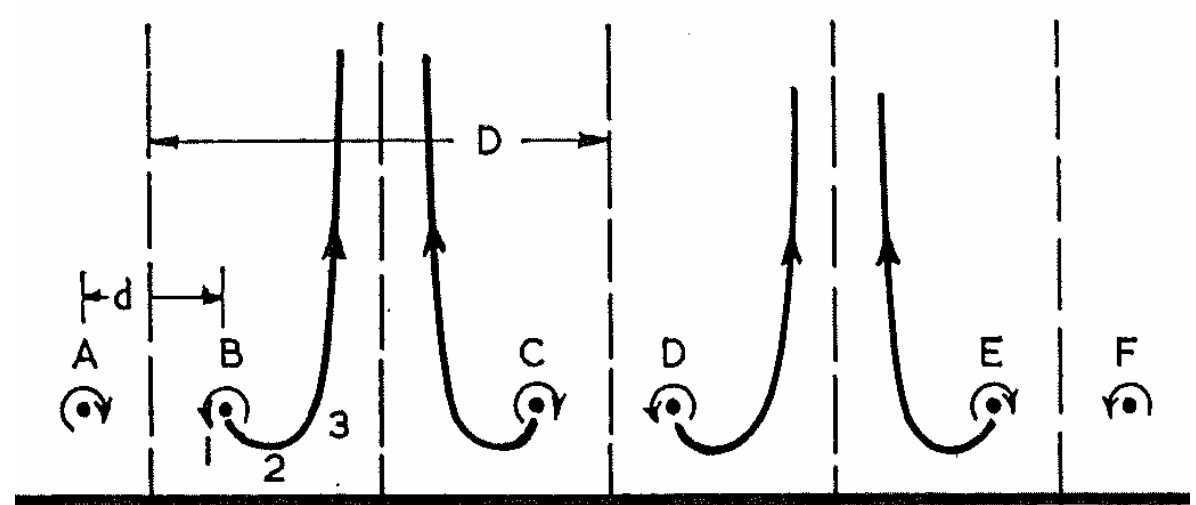

Figure 39: movement of the vortex cores projected on the crossflow plane as the vortices convect downstream for $D / d>2$, numbers indicate different stages in the movement; 1 . A and B transport one another towards the surface; 2 . B and $C$ vortexes move closer together; 3 . B and $C$ transport one another away from the surface (taken from Pearcey 1961).

If now it is assumed that indeed the micro-ramps generate streamwise vortices with a common flow up downstream of the vertex as suggested by figure 36 and that the locations of the mean vorticity streaks can serve as an indication for the positions of the streamwise vortices, it seems that in case of the staggered configuration, the ratio $\mathrm{D} / \mathrm{d}$ is larger than in case of the single row configuration and that the observed greater influence of the staggered configuration on the flow close to the surface is consistent with the description of the vortex motion as described by Pearcey (1961). 


\subsection{Instantaneous flow organization}

\subsubsection{Undisturbed interaction}

Contourplots of the instantaneous velocity fields of the undisturbed interaction at $y / \delta=0.1$ and $y / \delta=0.6$ are depicted in the left columns of figure 40 and figure 41 respectively. Within each column of figures the streamwise, spanwise and wall-normal velocity fields are shown from top till bottom. In the plots of the mean streamwise velocity, the instantaneous position of the sonic line, determined using equations in section 5.2, is depicted as well. The velocity fields in each column correspond to a single snapshot that is representative for those found in the data set.

As can be seen from figure 40a and figure 41a the instantaneous flow shows significant variations in streamwise velocity, ranging from $0.6 \mathrm{U}_{\infty}$ to $0.9 \mathrm{U}_{\infty}$ at $\mathrm{y} / \delta=0.1$ and from $0.8 \mathrm{U}_{\infty}$ to $1.0 \mathrm{U}_{\infty}$ at $\mathrm{y} / \delta=0.6$. This smaller range of velocities at a larger distance from the wall is consistent with Ganapathisubramani et al. (2007) and Humble et al. (2008). The variations in streamwise velocity appear to be organized in streamwise streaks of low and high-speed fluid. These streaks are randomly distributed in space as they have different positions each snapshot and disappear on the mean. A similar organization of supersonic boundary layers was reported by Ganapathisubramani et al. (2006) and Elsinga (2008), using planar and topographic PIV respectively. The organization is also consistent with results found in incompressible boundary layers using hot-film/wire anemometry (Kim and Adrian 1999; Hutchkins and Marusic 2007) and planar PIV (Tomkins and Adrian 2003; Ganapathisubramani et al. 2003).

From figure $40 \mathrm{a}$ it can be seen that at $\mathrm{y} / \delta=0.1$ the streamwise streaks, are not only coherent in the boundary layer, but also seem to determine the spanwise organization of the interaction. This idea is further investigated by taken the taking the location of a velocity contour (e.g. $0.4 \mathrm{U}_{\infty}$, yellow) as a proxy for the onset of the interaction; an approach that was also followed by Ganapatisubramani et al. (2007). Note that the use of such a criterion is ill-stated because the position of the reflected shock is characterised by a decrease in velocity and not by attaining a certain threshold. Furthermore, the flow in the field of view is largely subsonic making the inference of the reflected shock's position problematic. Nevertheless the approach is sufficient for the present purposes.

Thus, taking the location of a velocity contour (e.g. $0.4 \mathrm{U}_{\infty}$, yellow) as a proxy for the onset of the interaction, it seems that downstream of a low-speed streak in the upstream boundary layer, the interaction in general commences at a more upstream position (e.g. at $\mathrm{z} / \delta=-0.4$ ). Downstream of a high-speed streak the opposite effect occurs: the onset of the interaction is push downstream (e.g. at $\mathrm{z} / \delta=-0.8$ ). This relation between the velocity in the incoming boundary layer and the location of the onset of the interaction was also found by Beresh et al. (2002) Ganapathisubramani et al. (2007) and Humble et al. (2008). In section 5.5 the relation will be father investigated by means of a statistical analysis.

Figure 40a also shows that when the onset of the interaction is pulled upstream, the fluid attains a lower minimum velocity in the interaction and recovers more slowly (e.g. at $\mathrm{z} / \delta=-$ 0.4). The opposite is true if the onset of the interaction is pushed upstream. In that case the fluid in less decelerated throughout the interaction and recovers more quickly (e.g. at $\mathrm{z} / \delta=-$ 0.8). Again this is consistent with Ganapathisubramani et al. (2007) and Humble et al (2008). 
It is remarked that although on the mean no reversed flow occurs, patches of reversed flow can instantaneously be seen downstream of low-speed streaks at $y / \delta=0.1$ (see figure 40a). The largest patches have a length in the order of a boundary layer thickness and are typically twice as long as they are wide. The minimum observed streamwise velocity in the patches is $0.2 \mathrm{U}_{\infty}$. Their location and the percentage of the time that reversed flow occurs will be investigated thoroughly in section 5.5.

At $y / \delta=0.6$ the same behaviour of the streamwise velocity as at $y / \delta=0.1$ could be observed, but in general streamwise elongated regions can less often be observed. This is consistent with tomographic PIV results of Humble et al. 2008 who found results suggesting that elongated low speed regions do not extend much farther than $y / \delta=0.6$ and observations of Ganapathisubramani et al. (2007), who reported that the presence of strips of uniform momentum in the incoming boundary layer at $\mathrm{y} / \delta=0.7$ is not as common compared to measurements at $\mathrm{y} / \delta=0.2$.

On the occasions that streamwise elongated regions can be found in the incoming boundary layer, it seems they determine the interaction to a lesser extent than at $y / \delta=0.1$, which is in agreement with results from a PIV study from Beresh et al. (2002). It should be noted that the length of streamwise excursions of the onset of the interaction is much larger at $y / \delta=0.1$ than at $y / \delta=0.6$ as was already observed from the mean flow organization. This is consistent with Dupont et al. (2006) who found that the intermittent region becomes smaller further away from the wall. Also discrete PDFs of the shocks location obtained by Humble et al. (2008) are in agreement with this observation.

From the instantaneous spanwise velocity field at $y / \delta=0.1$ it can be seen that the spanwise velocity is far less coherent along both the streamwise and spanwise directions, consistent with Ganapathisubramani et al. (2006) and Humble et al (2007b). The spanwise velocity in the incoming boundary varies within the range $-0.08<\mathrm{u} / \mathrm{U}_{\infty}<0.08$. At $\mathrm{y} / \delta=0.6$ the length scale of the spanwise velocity variations does not appear to be different from that at $y / \delta=0.1$ (compare figure 41d and figure 40d). Note that the magnitude of spanwise velocity at $y / \delta=$ 0.1 becomes about twice as large in the interaction region and does not appear to decrease again within the field of view (see figure $40 \mathrm{~d}$ ), while at $\mathrm{y} / \delta=0.6$ no increase in magnitude can be observed (figure 41d). The increase in magnitude is consistent with results found by Humble et al. (2008).

Figure $40 \mathrm{~g}$ shows that the characteristic lengths of the wall-normal velocity at $\mathrm{y} / \delta=0.1$ appears to be significantly smaller than those of the other two velocity components. Moreover, the deflection of the flow away from the wall due to the interaction occurs in very compact regions that seem to be alternated by regions in which the fluid is directed parallel to or towards the wall. Occasionally even compact regions can be found in which the flow moves towards the wall with a velocity greater than $0.3 \mathrm{U}_{\infty}$. As the occurrence of such regions was found to be highly dependent on data processing parameters, suggesting that at least some of them might be spurious vectors, caution should be taken when relating the presence of these particular regions to the interaction's phenomenology.

Downstream of the interaction, the same compact organization can be seen but with typically lower wall-normal velocities than in the interaction region. Comparing figure $40 \mathrm{~g}$ and figure $41 \mathrm{~g}$ it can be seen that the characteristic length scale of the wall-normal velocity in streamwise and spanwise direction increases away from the wall, which is a well known result 
in literature (see e.g. Smith and Dussauge 2006). Furthermore, the deflection of the flow due to the interaction appears to become more coherent.

Note that the instantaneous behaviour of the undisturbed interaction is very much like the its mean behaviour induced by the micro-ramps: downstream of the apices of the micro-ramps, where on the mean low-speed streak were located, the interaction could be seen as well to commence at a more upstream position (if a velocity contour would have been used as a proxy for the onset of the interaction) and to lead to a lower minimum streamwise velocity and a slower recovery of the boundary layer. 


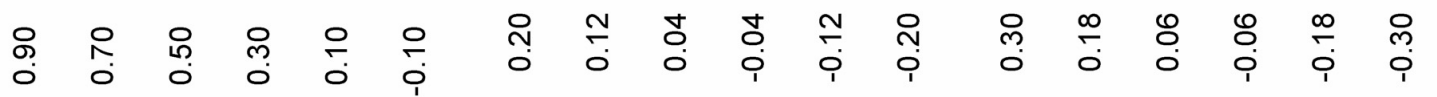
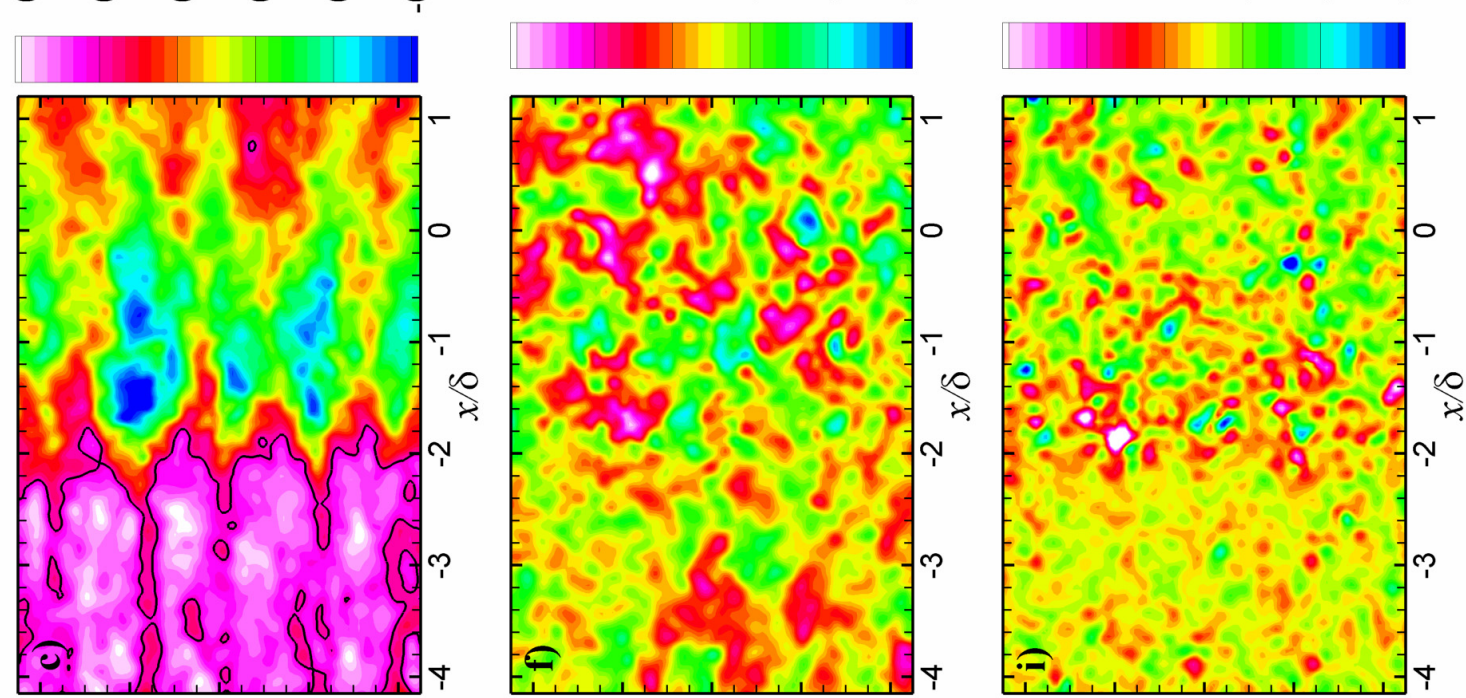

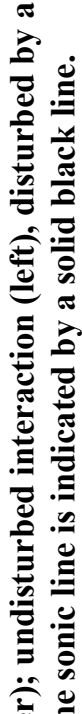
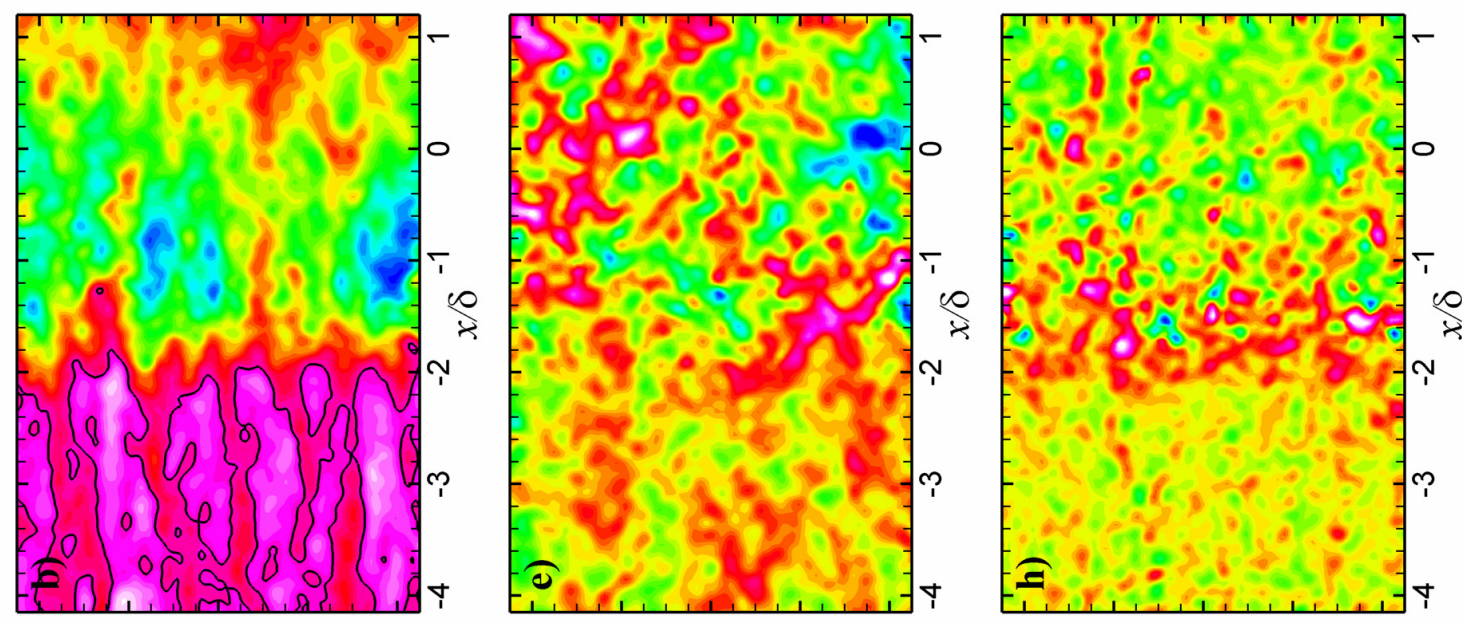

(ี)

䔾

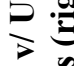

胥

它䓪

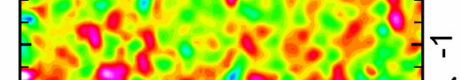
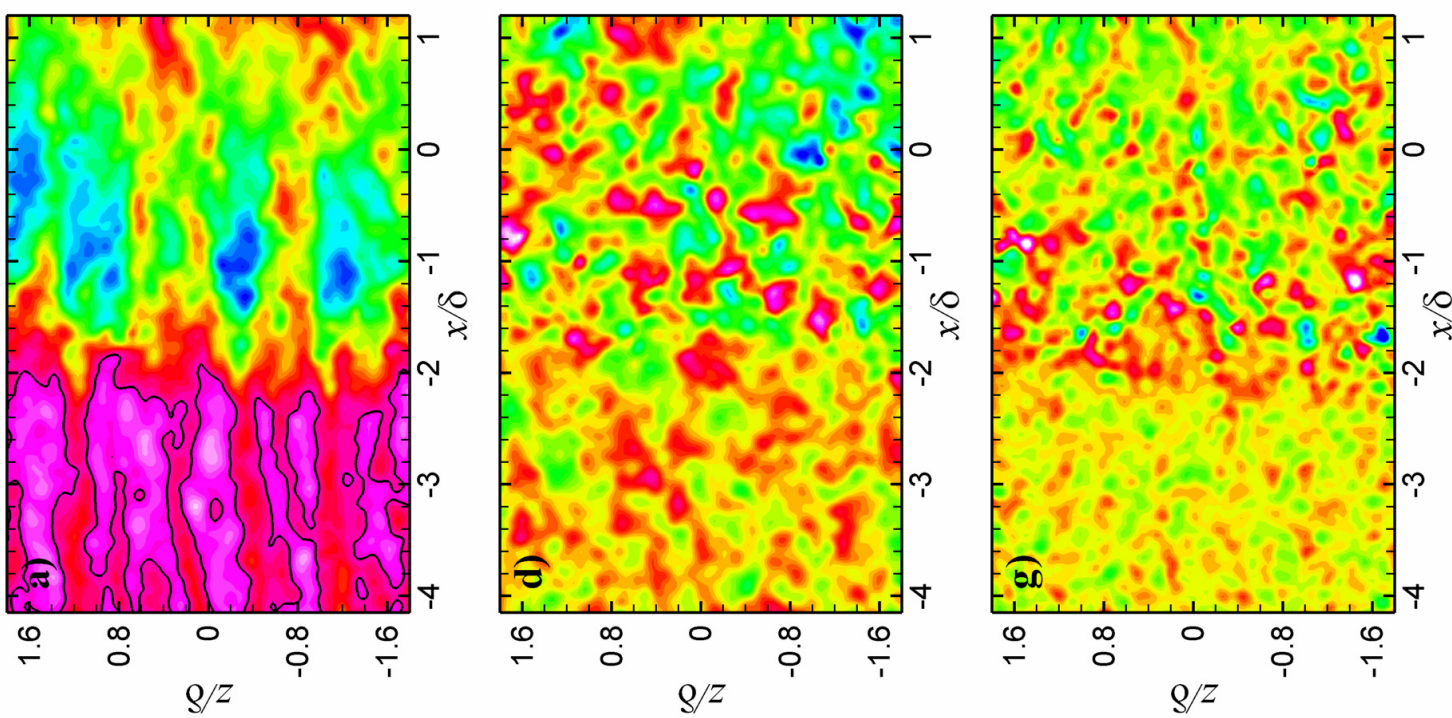

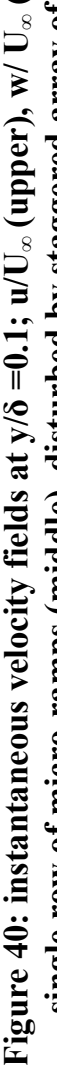




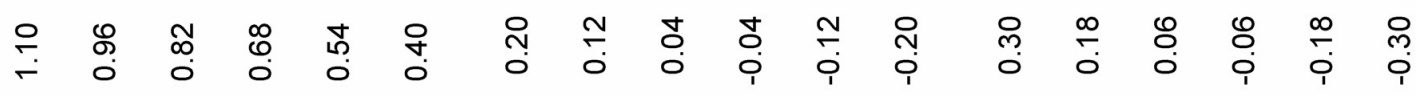
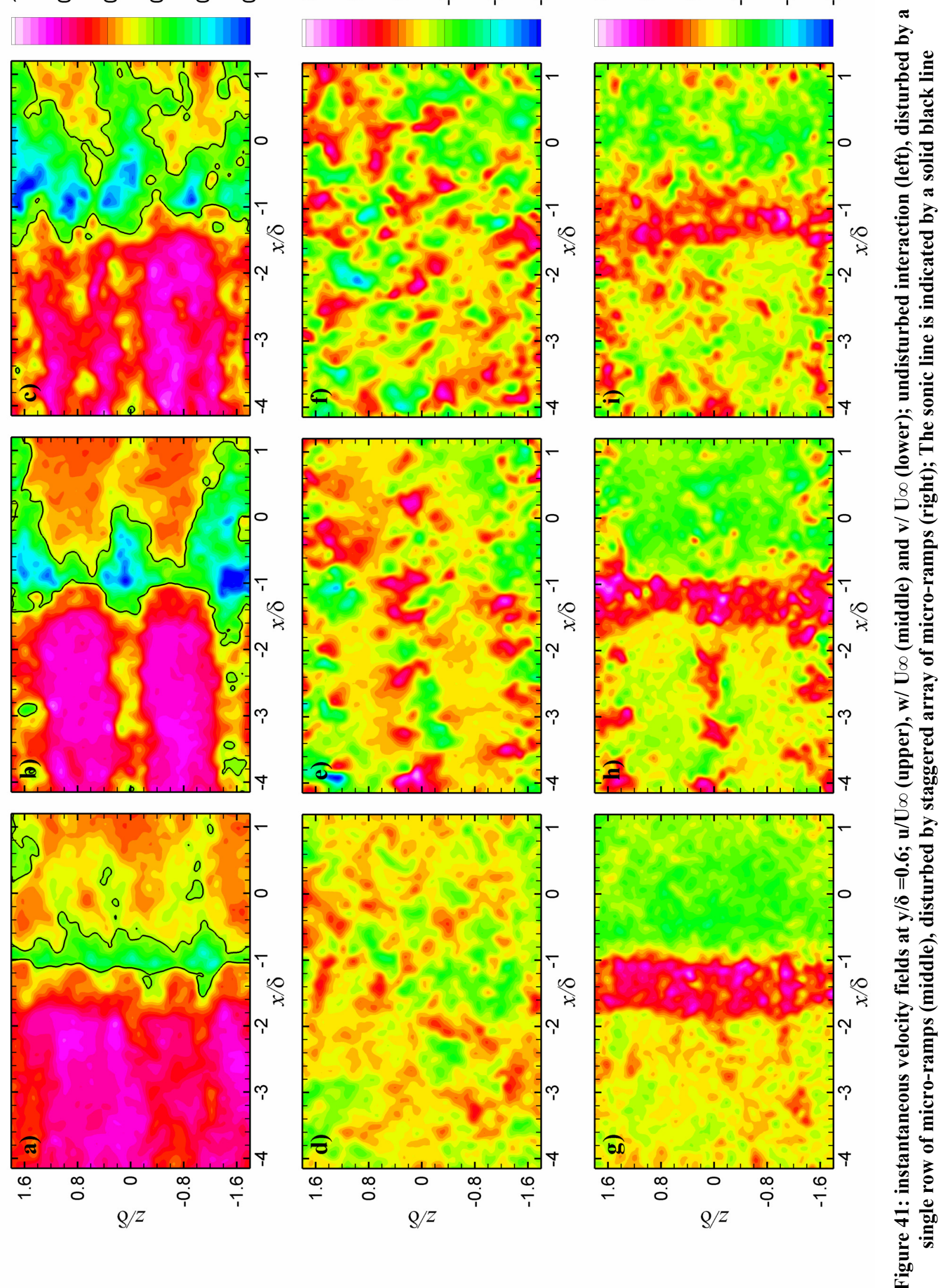


\subsubsection{Perturbed interactions}

Contourplots of the instantaneous velocity fields of the interactions downstream of a single row and downstream of a staggered array of micro-ramps are depicted in the middle columns and right columns respectively of figure $40(\mathrm{y} / \delta=0.1)$ and figure $41(\mathrm{y} / \delta=0.6)$. The figures are plotted in the same way as those of the undisturbed interaction and the depicted snapshots are representative for those found in the data set.

The streamwise instantaneous velocity fields (upper rows figure 40 and figure 41) downstream both configurations suggest the same interaction dynamics as those of the undisturbed interaction: downstream of a low-speed region in the incoming boundary layer, the onset of the interaction, based on a velocity contour, is located more downstream, the minimum attained velocity is lower and the recovery of the boundary layer is slower than downstream of a high-speed streak.

From observations of a large number of snapshots, it was found that downstream micro-ramps the positions of the streamwise streaks do not so much change each snapshot as in the undisturbed interaction. Instead they appear to be more fixed in space. This is especially true downstream of the single row configuration at $y / \delta=0.6$ and downstream of the staggered configuration at $\mathrm{y} / \delta=0.1$. In contrast, downstream of the single row configuration at $\mathrm{y} / \delta=0.1$ such an effect could not be found from visual inspection.

This is consistent with observations made regarding the occurrence of reversed flow. For both configurations, the patches of reversed flow look much alike the patches observed in the undisturbed interactions in terms of their typical size and velocity. In case of the staggered array however reversed flow seems to occur more often downstream of vertex locations than elsewhere, while in case of the single row no such behaviour could be observed from instantaneous velocity fields. A statistical analysis of the effect of micro-ramps on the occurrence of reversed flow will be described section 5.5 .

The instantaneous spanwise and wall-normal velocity fields downstream of the perturbed interactions at $y / \delta=0.1$ do not show any particular difference in organization with respect to the undisturbed interaction. At $y / \delta=0.6$ however, compact regions with coherent motions can be found in the incoming boundary layer along streamwise lines downstream of vertex locations from both spanwise and wall-normal velocity fields (figure 41e, f). Downstream of the interaction the regions with coherent motions do no longer appear to be positioned along streamwise lines.

\subsubsection{Vorticity distribution}

In order to make statements regarding the instantaneous vortex organization, the vorticity distributions at $\mathrm{y} / \delta=0.1$ and $\mathrm{y} / \delta=0.6$ are shown in figure 42 and figure 43 respectively. The out-of-plane vorticity is determined by inserting instantaneous velocities in equation (16) in (section 5.2.3). The depicted snapshots are the same as those depicted in figure 42 and $i$. Note that the range of magnitudes used at $\mathrm{y} / \delta=0.6$ is twice as small as the one used at $\mathrm{y} / \delta=0.1$.

Figure $42 \mathrm{a}$ shows that at $\mathrm{y} / \delta=0.1$ like the streamwise velocity, the vorticity in the undisturbed boundary layer is organized in streamwise streaks. Throughout the interaction, the vorticity increases significantly. Both observations are in agreement with Ganapathisubramani et al. (2007). Downstream of the interaction, the characteristic streamwise length scale seems to be increased with respect to the upstream boundary layer. 
Comparing figure $42 \mathrm{a}, \mathrm{b}$ and $\mathrm{c}$, it can be seen that at $\mathrm{y} / \delta=0.1$ the vorticity distributions of the undisturbed interaction and the perturbed interactions look alike.

Figure 43 a shows that at $y / \delta=0.6$ the vorticity in the undisturbed incoming boundary layer is much less coherent in streamwise direction than at $y / \delta=0.1$ and the spanwise characteristic length scale seems to be increased, which is consistent with results found by Ganapathisubramani et al. (2006) and by Elsinga (2008). Comparing figure 43a and figure $41 \mathrm{a}$, it seems that the highest vorticity is located at relatively low-speed regions in the boundary layer, which is in agreement with Elsinga (2008). Like at $y / \delta=0.1$ amplification of the vorticity can be seen in the interaction region.

Comparing figure $43 \mathrm{a}$ and figure $43 \mathrm{~b}, \mathrm{c}$ it can be seen that unlike at $\mathrm{y} / \delta=0.1$ the vorticity distribution in the perturbed flow at $\mathrm{y} / \delta=0.6$ is very different from that in the unperturbed flow. In figure $43 \mathrm{~b}$ distinct vortex structures can be seen along three streamwise lines downstream of the vertex locations in the single row configuration. In case of the staggered array the number of streamwise lines along which counter-rotating vortices are positioned has increased to five, conform to the number of micro-ramps (see figure 43c) and as a result the instantaneous vorticity distribution looks more complex.

For both configurations the vorticity within the interaction region does not seem to increase as at $y / \delta=0.6$ but the density of vortical structures appears to increase in both streamwise and spanwise sense. Downstream of the interaction, vortical structures are less well defined and more randomly distributed. It should be noted that in case of the staggered array, the number of vortical structures downstream of the interaction is significantly larger than in case of the single array, suggesting a more pronounced mixing at $\mathrm{y} / \delta=0.6$. 

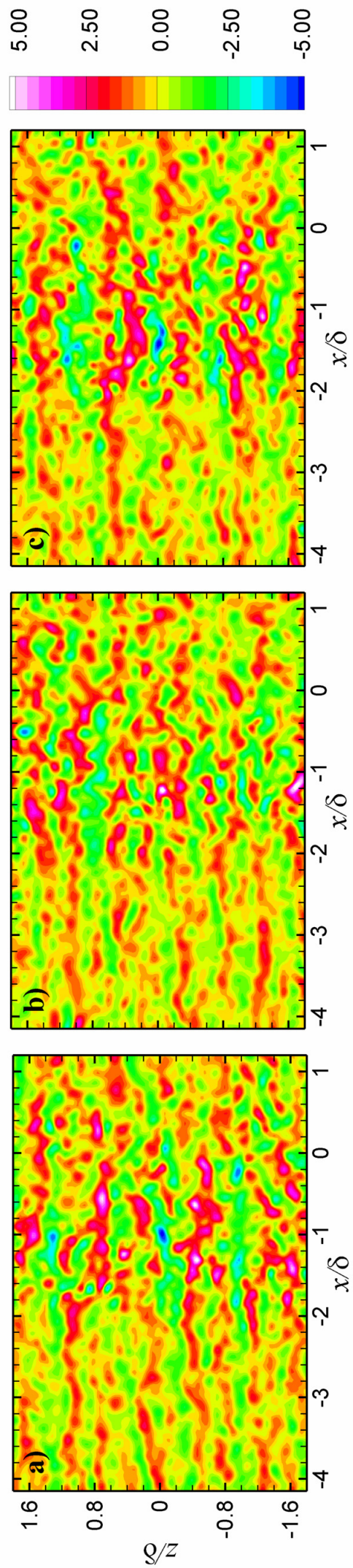

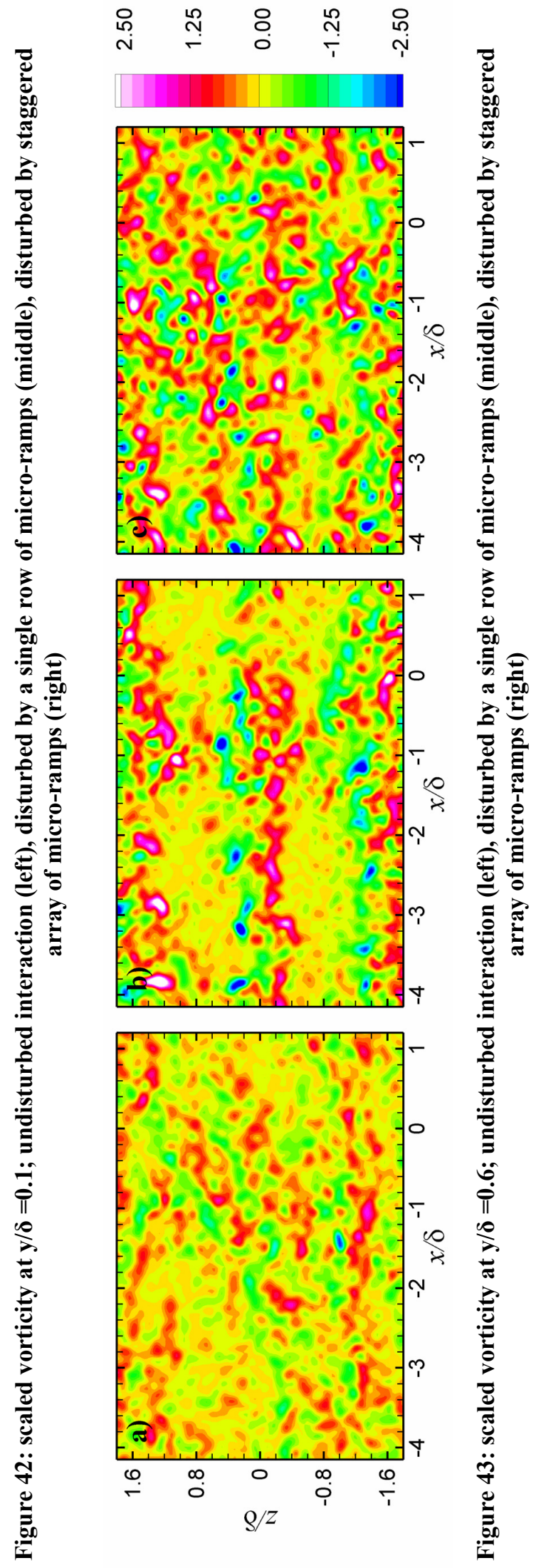




\subsubsection{Structure of the incoming boundary layer}

In previous sections it was found that the instantaneous three-dimensional organization of the interaction seems to be influenced to a large extent by spanwise velocity variations in the incoming boundary layer. For this reason an investigation of the instantaneous structure of the incoming boundary layer and the effect of the micro-ramps thereon, will be helpful to gain further insight in the effect of micro-ramps on the instantaneous structure of the interaction.

Figure $44 \mathrm{a}, \mathrm{b}$ show instantaneous streamwise velocity fields at $\mathrm{y} / \delta=0.1$ of the undisturbed boundary layer without shock interaction and the boundary layer perturbed by a single row of micro-ramps without shock interaction respectively. The depicted snapshots are representative for those found in the data set.

Consistent with previous observations from velocity and vorticity plots, figure 44 shows that the variations in instantaneous streamwise velocity within both boundary layers are organized in streamwise streaks of low and high-speed fluid. From visual inspection of many realizations it was found that the range of velocities within the boundary layer is comparable for the undisturbed and perturbed boundary layers. This suggests that the major effect of the micro-ramps at $y / \delta=0.1$ is to determine to some extent the spanwise locations of the streaks: as was already described in section 5.3.2: downstream of vertex locations low-speed streaks seem to be more likely to occur, accounting for the presence of low-speed regions at these locations in the mean velocity fields. The opposite is true for spanwise regions away from the vertices.

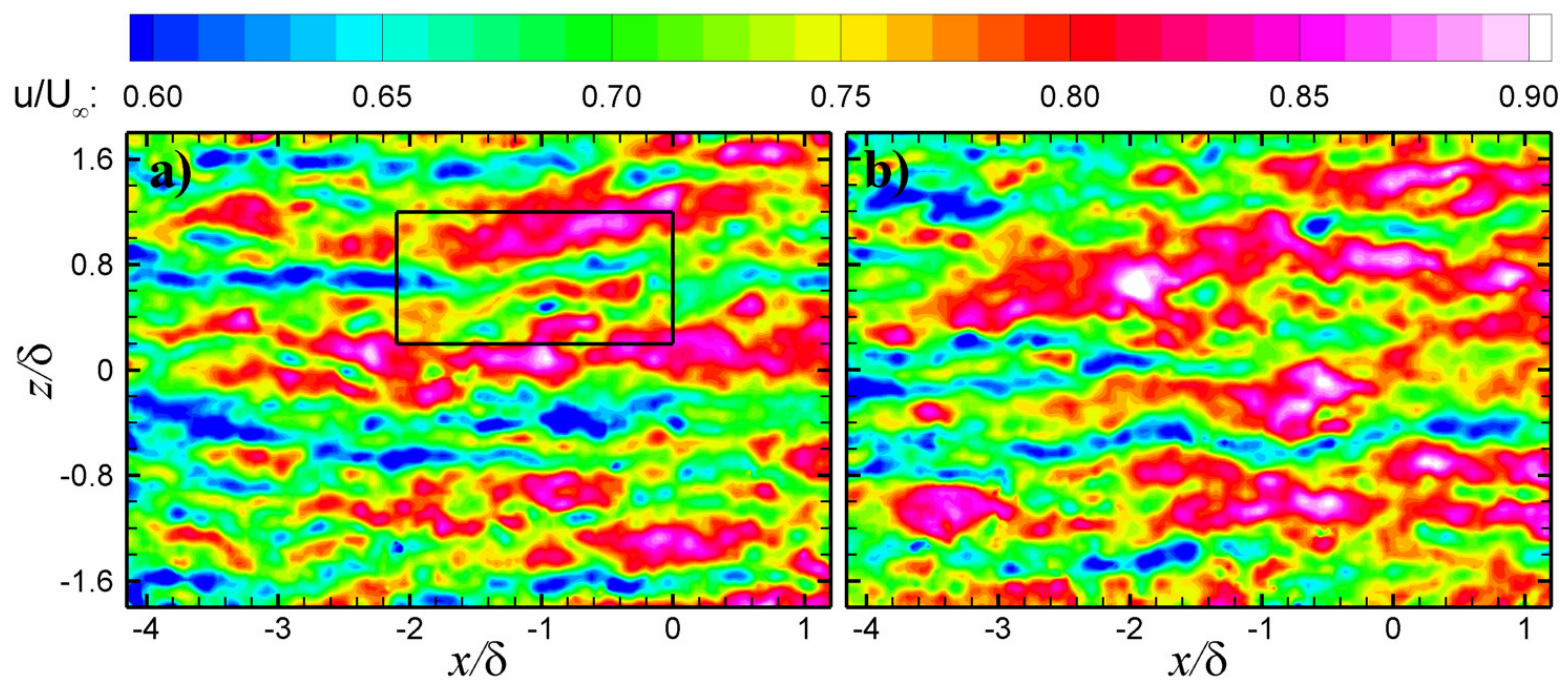

Figure 44: instantaneous streamwise velocity fields at $y / \delta=0.1$; undisturbed boundary layer (left), boundary layer perturbed by a single row of micro-ramps (right); the black rectangle encloses a structure that will be zoomed into later.

Figure 44 shows that the high- and low-speed streaks are at least as long as the field of view. Remember from section 2.4 that the existence of streaks in supersonic boundary layers with lengths up to $40 \delta$ has been demonstrated by other experiments (Ganapathisubramani et al. 2006). By dividing the spanwise length of the field of view by the number of streaks that typically can be observed, the width of the streaks was estimated. At $y / \delta=0.1$ this procedure returned a streak width of $0.3-0.4 \delta$, which is very close the range of $0.25-0.4 \delta$ in the region $\mathrm{y} / \delta=0.15-0.47$ reported by Elsinga (2008), the range of $0.25-0.5 \delta$ at $\mathrm{y} / \delta=0.2$ found by 
Ganapathisubramani et al. (2006) and widths of about $0.5 \delta$ found from DNS data by Ringette et al. (2008).

In order to look more closely into the organization of the flow within the streaks, a zoom of the area enclosed by the black rectangle in figure $44 \mathrm{a}$ is depicted in figure 45 . The upper figure shows the vorticity and the lower the vorticity streamwise velocity. The black circles enclose some, but certainly not all, locations of increase vorticity. The size of the circles was chosen arbitrary. To illustrate the existence of vortical structure at these locations, vectors are shown in a convective frame of reference of the mean velocity in streamwise direction $\left(0.73 \mathrm{U}_{\infty}\right)$.

Comparing the locations of the black circles in the upper and the lower figure it can be seen that regions of increased vorticity typically are located at the interface between the low-speed and high-speed streaks. This observation is in agreement with Elsinga (2008), Ringuette et al. (2008) and Humble et al. (2008) and the hairpin vortex packet model of incompressible turbulent boundary layers (Kim and Adrian, 1999; Tomkins and Adrian 2003), which asserts that hairpin-like structures induce negative velocity fluctuations between their legs and positive fluctuations on either side of their legs.

\section{$\omega$}

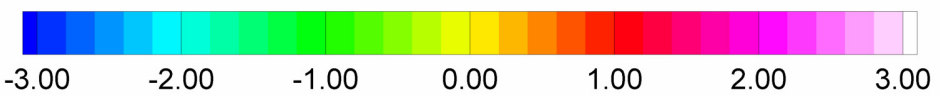

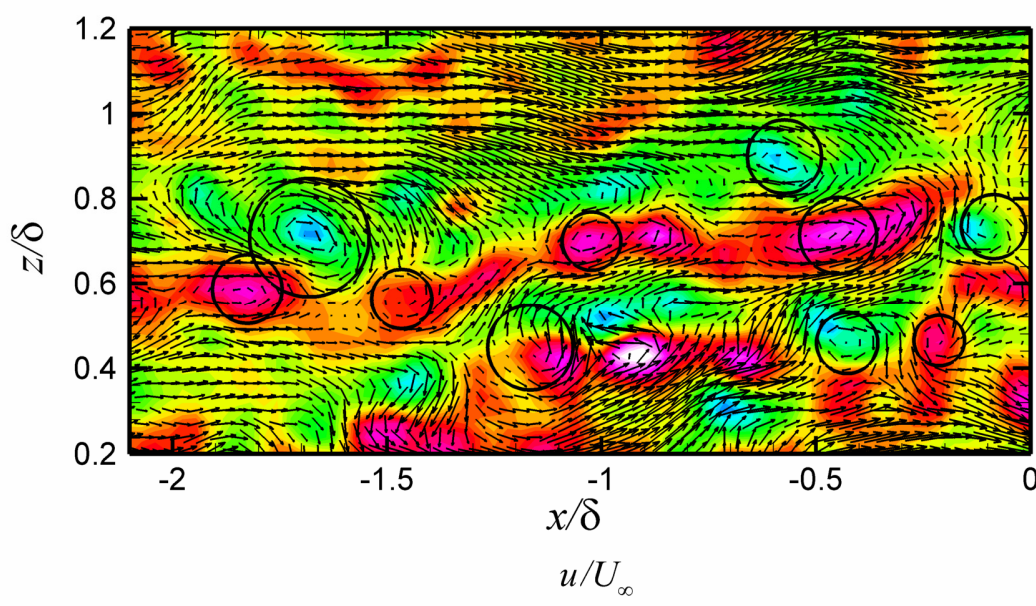

\begin{tabular}{lllllll}
\hline 0.60 & 0.66 & 0.72 & 0.78 & 0.83 & 0.89 & 0.95
\end{tabular}

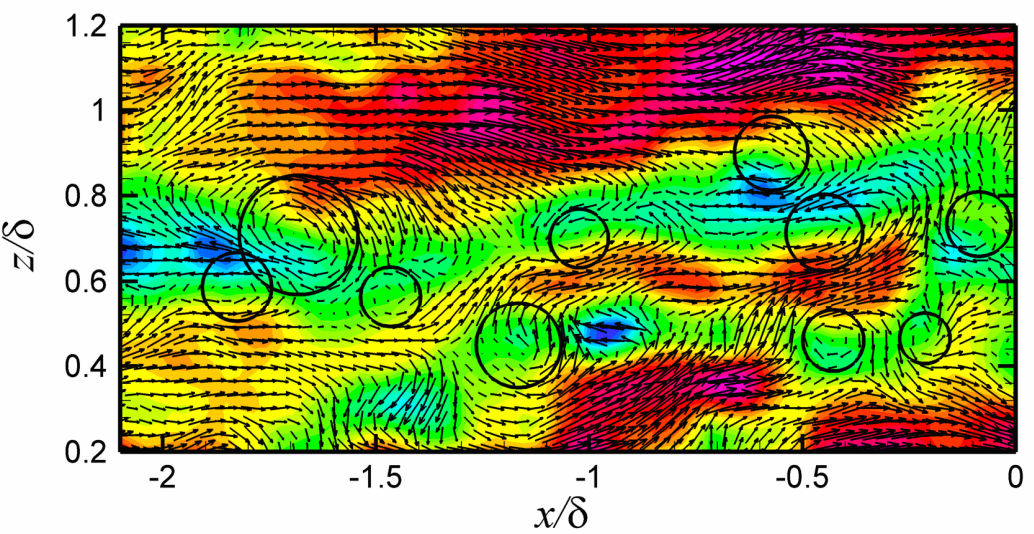


Figure 45: zoom of the area enclosed in the previous figure; vorticity (upper) and streamwise velocity (lower); vectors are shown in a convective reference frame of the mean streamwise velocity $\left(0.73 \mathrm{U}_{\infty}\right)$; the black circles denote several locations of increase vorticity, the size of the circles is arbitrary.

Figure $46 \mathrm{a}$ and $\mathrm{b}$ show the instantaneous streamwise velocity fields of the undisturbed boundary layer and boundary layer perturbed by a single row of micro-ramps at $y / \delta=0.6$. As mentioned earlier, the presence of low-speed streaks in the undisturbed boundary layer at this distance from the wall is not as common as at $y / \delta=0.1$. Typically 4-6 streaks could be observed. Dividing the spanwise length of the field by this number gives a streak width of 0.4-0.6 $\delta$. This increase in streamwise length scale with respect to $y / \delta=0.1$ is consistent with results found by Ganapathisubramani et al. (2006) and by Elsinga (2008). At $y / \delta=0.6$ it was found that the location of low-speed streaks typically coincided with regions in which fluid moves away from the wall.

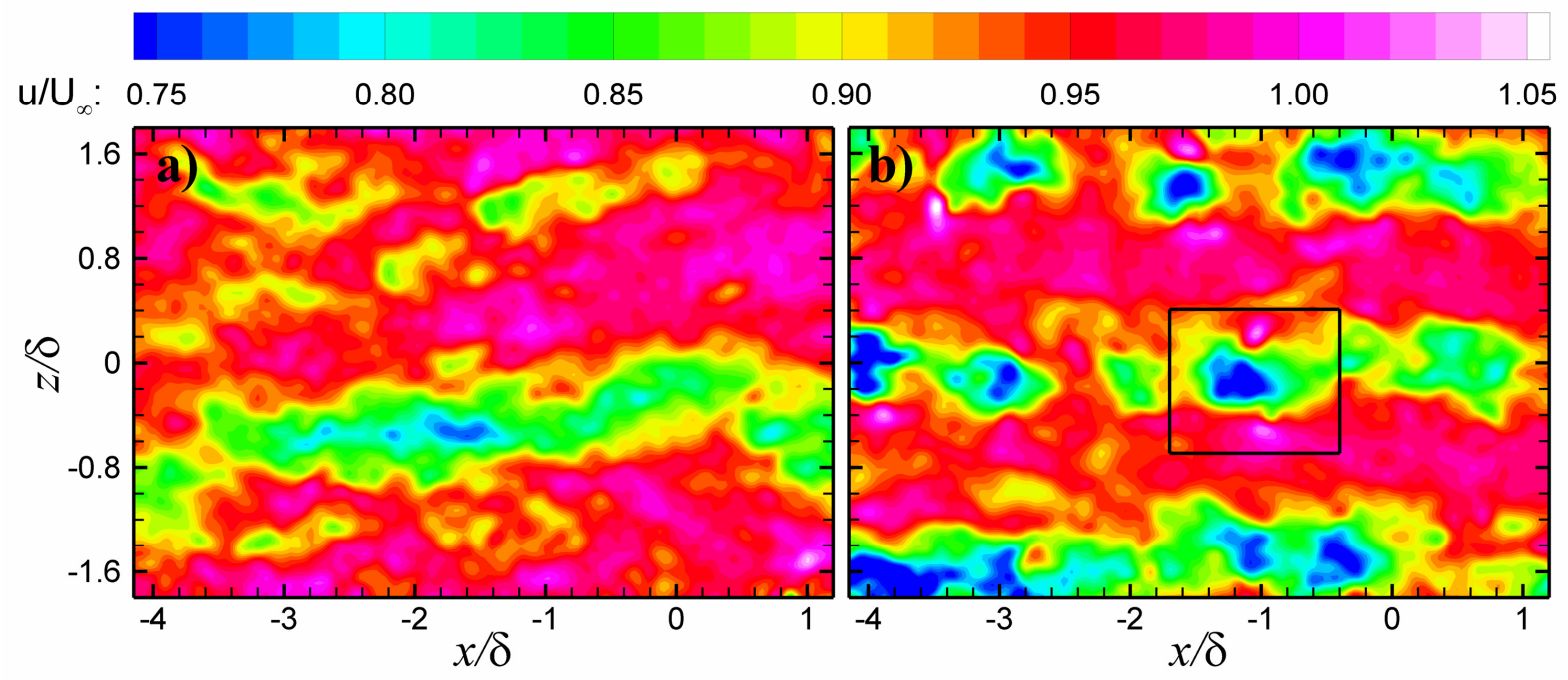

Figure 46: instantaneous streamwise velocity fields at $y / \delta=0.6$; undisturbed boundary layer (left), boundary layer perturbed by a single row of micro-ramps (right); the black rectangle encloses a structure that will be zoomed into later.

Comparing figure $46 \mathrm{a}$ and figure $46 \mathrm{~b}$ it can be seen that unlike at $\mathrm{y} / \delta=0.1$ the flow organization within the low-speed regions in the perturbed flow at $y / \delta=0.6$ is very different from that in the unperturbed flow. While in the undisturbed boundary layer a low-speed streak looks just like an elongated region, in the perturbed flow, the low-speed regions downstream of the micro-ramps appear to consist of multiple structures that pass by at regular intervals of about one boundary layer thickness.

One of these structures is depicted in figure 47. The depicted region in the figures corresponds to the region enclosed by the black rectangle in figure $46 \mathrm{~b}$. Depicted vectors were obtained after subtracting the local mean streamwise velocity $\left(0.85 \mathrm{U}_{\infty}\right)$. The vorticity plot shows that the convected structures consist of two counter-rotating vortices. The wall-normal velocity field suggests that the vorticity also has a component in the streamwise direction (see figure 47c). It is therefore speculated that the instantaneous vortices are inclined with respect to the wall. In this organisation, the streamwise vorticity component would account for the streamwise vortices that were observed in the mean flow organisation, while the out-of-plane component attributes to a large extent to the reduction in mean streamwise velocity downstream of the micro-ramps. 

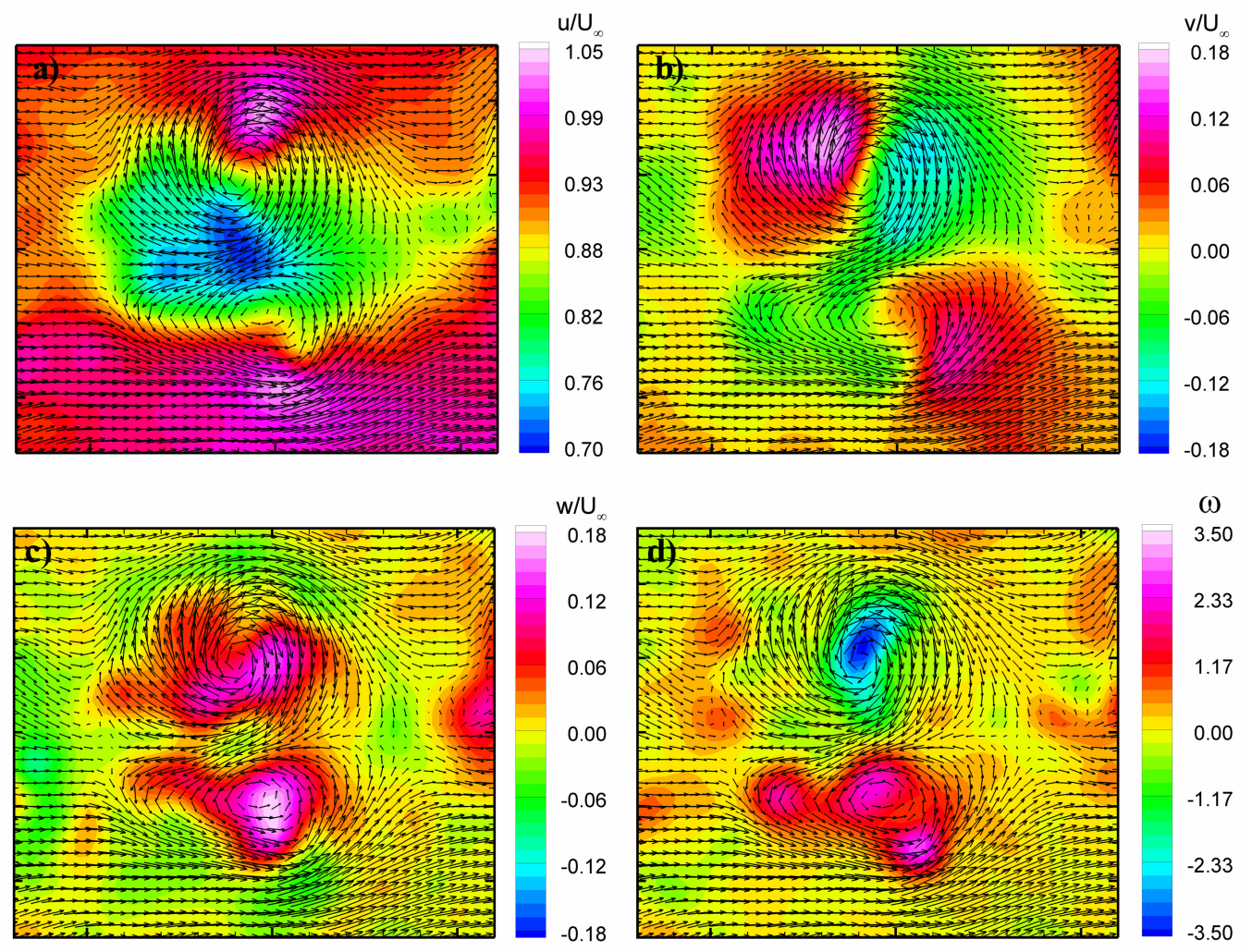

Figure 47: zoom of a vortex at $\mathbf{y} / \delta=0.6$ downstream of a micro-ramp; instantaneous streamwise velocity (top left), instantaneous spanwise velocity (top right), instantaneous wall-normal velocity (bottom left) and instantaneous vorticity (bottom right); vectors are shown in a convective reference frame of the local mean streamwise velocity $\left(0.73 \mathrm{U}_{\infty}\right)$

The vortex signature shown in figure 47 and the speculation on the inclination of the vortex pair with respect to the wall, is reminiscent of conditional eddies obtained by Tomkins and Adrian (2003) and by Elsinga (2008). The latter is depicted below in figure 48. Identifying the vortex pair as a cross-cut of a hairpin vortex would be premature given the amount of present evidence and more research would be needed to test this hypothesis. Nevertheless, the reader is invited to note the similarities with the vortex pair shown in figure 47 both in terms of length scales and in flow organization.

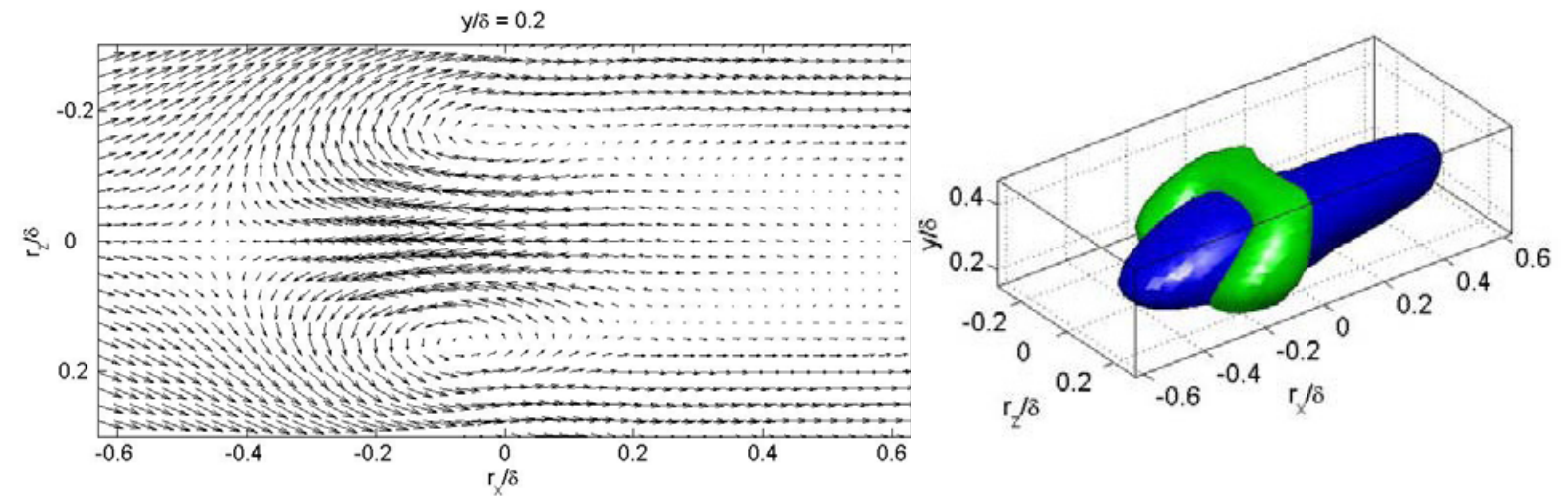

Figure 48: conditional eddy given a negative spanwise swirling event at $y / \delta=0.35$; velocity vector plot in the $x, z$ cross-section (left) and three-dimensional visualization (right) where the green contour denotes the vortex and the blue contour the induced low speed zone; velocity vectors are relative to the local average velocity; taken from Elsinga (2008). 
Tufo et al. (1999) investigated hairpin vortices in the wake of a hemispherical roughness element in a low-speed turbulent incompressible boundary layer using direct numerical simulations (DNS). Their results are depicted below in figure 49. As can most clearly be seen in lower figure their results a movement of both heads and tips of the vortices away from the plate. Although, the presence of hairpin vortices downstream of micro-ramps as well as generalization of their movement away from the wall to compressible boundary layers are subjects of tentative speculations, the results of Tufo et al. (1999) indicate that the presence of instantaneous hairpin vortices does not preclude the vortex migration as hinted by the present oil-flow visualizations and further discussed in the section on the mean results.
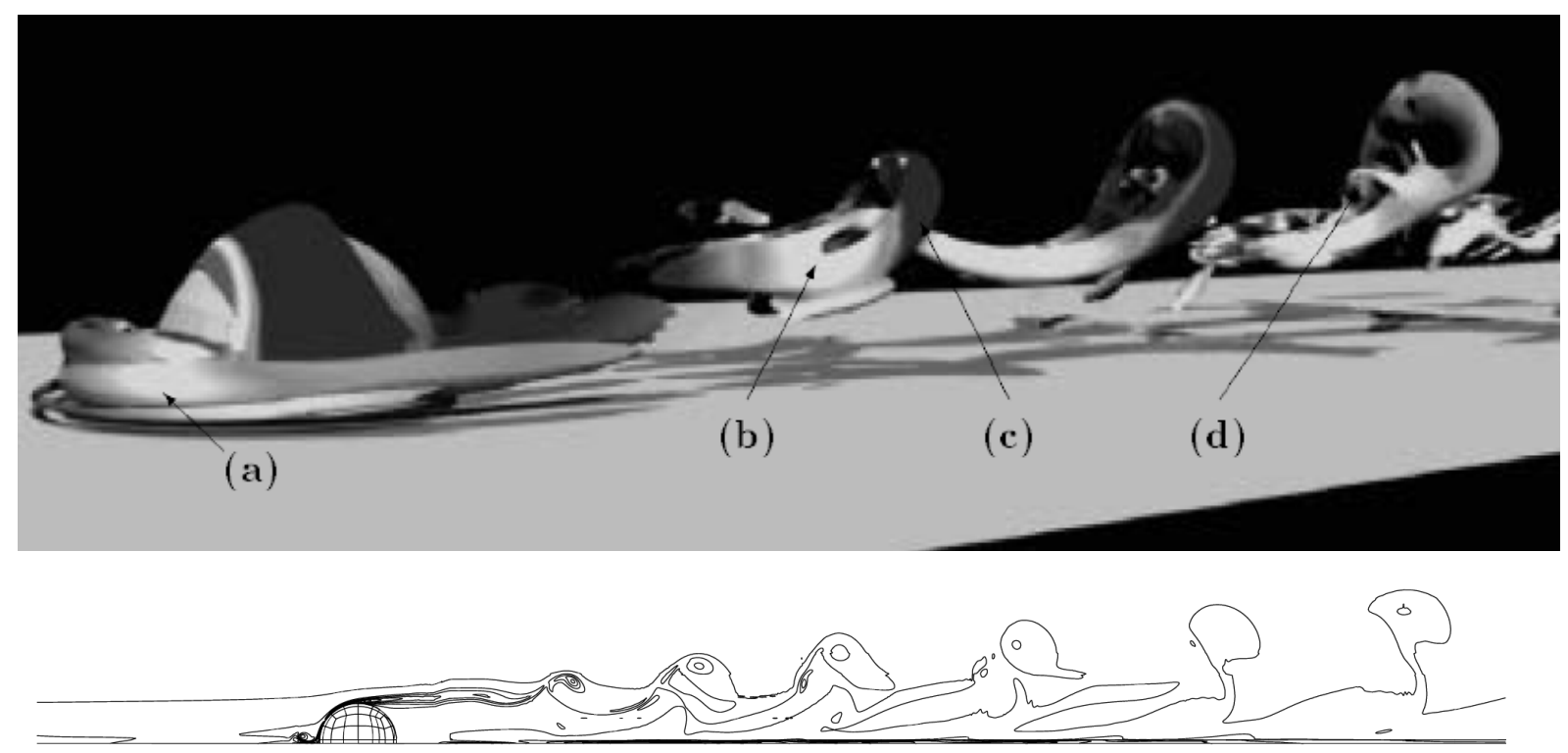

Figure 49: hairpin vortex structures downstream of a hemispherical roughness element; features indicated by a,b,c and d will not be discussed in present thesis (upper); contours of spanwise vorticity along symmetry plane (lower). From Tufo et al. (1999). 


\subsection{Turbulent flow organization}

\subsubsection{Undisturbed interaction}

Spatial distributions of the turbulence intensity are depicted in the left columns figure 50 and figure 51. Within each column the streamwise $\langle$ ' $>$, spanwise $\langle$ ' $>$ and wall-normal $\langle$ w' $>$ RMS velocities, scaled by the freestream velocity are shown from top till bottom. The contour scales are chosen such to allow good comparison between the three interactions and are not suited for comparison between the three different components.

It can be seen that turbulence levels start to increase at the same streamwise location where the flow starts to decelerate. At $y / \delta=0.1,\left\langle\mathrm{u}^{\prime}>\right.$ and $\langle\mathrm{w}$ ' $>$ reach maximum values of about $0.20 \mathrm{U}_{\infty}$ and $0.12 \mathrm{U}_{\infty}$ respectively. Further away from the wall the maximum intensities of $<\mathrm{u}^{\prime}>$ and $\langle$ w' $>$ are about twice as low. These values are consistent with results found by Humble et al. (2007). The increase of $\left\langle\mathrm{v}^{\prime}\right\rangle$ in the interaction region is much smaller. It reaches a maximum of $0.07 \mathrm{U}_{\infty}$ at $\mathrm{y} / \delta=0.1$ and of $0.04 \mathrm{U}_{\infty}$ at $\mathrm{y} / \delta=0.6$.

At $y / \delta=0.1$ the turbulence in wall-normal and streamwise directions subsequently decreases downstream of the point where the flow becomes parallel to the wall, whereas the turbulence in spanwise direction remains relatively high for a longer streamwise distance and does not appear to decrease for about another two boundary layer thicknesses.

Note that at $y / \delta=0.6$ two peaks in turbulence can be identified in plots of both the streamwise and wall normal turbulence: one at $\mathrm{x} / \delta=-1.6$ and another at $\mathrm{x} / \delta=-1.0$. The most upstream peak is associated with the reflected shock and the second peak with the incident shock. The peak at $\mathrm{x} / \delta=-1.6$ is lower than the peak at $\mathrm{x} / \delta=-1.0$, which could partially be explained by the fact that the flow encounters the second disturbance before it is fully recovered from the first. In case of $<v '>$ only one region of increasing turbulence can be observed.

\subsubsection{Perturbed interactions}

The distributions of the turbulence intensities downstream of a single row of micro-ramps are depicted in the middle column of figure 50 and figure 51 in the same way as the undisturbed interaction in the left column.

Figure 51 shows that at $y / \delta=0.6$, the single row configuration induces a very clear spanwise organization of the turbulence distribution: downstream of the micro-ramps streamwise regions exist where the turbulence intensity is significantly increased with respect to the undisturbed interaction. In between these regions the turbulence intensity is lower than in the undisturbed case. Throughout the interaction the turbulence levels can be seen to increase. The obtained results at $\mathrm{y} / \delta=0.6$ are consistent with the previously found organization in vortical structures that are convected downstream of vertex locations. Closer to the wall at $y / \delta$ $=0.1$ effect of a single row configuration is significantly smaller with respect to that at $y / \delta$ $=0.6$ (compare middle columns figure 50 and figure 51 ). It only appears to have a noticeable effect on $\langle u$ ' $>$. The turbulence intensity of this component is lower downstream of the microramps in the incoming boundary layer, throughout the interaction and during the recovery of the flow. Note that this effect of the ramps is opposite to that at $y / \delta=0.6$.

As can be seen in the right column of figure 50 , the influence of the staggered array at $y / \delta=$ 0.1 is significantly larger than that of the single row, which is consistent with previous results. 
All turbulence components now show a spanwise organization. The turbulence levels in the interaction can be seen to be highest downstream of most upstream row of micro-ramps. In contrast we see that at $y / \delta=0.6$ the highest turbulence levels occur downstream of the most downstream row of micro-ramps. Note that this difference between the relative influence of both rows in consistent with previous results.

Overall, the relatively low turbulent regions in between vertex locations that were observed in case of the single-row have largely disappeared and as a consequence the effect of the staggered array on the turbulence intensity at $y / \delta=0.6$ is more uniform in spanwise direction than that of the single row (figure 51). For both configurations the highest intensities in the interaction region at $y / \delta=0.6$ can be found on the sides of the region where on the mean the lowest velocities were observed. 
꽁 둥

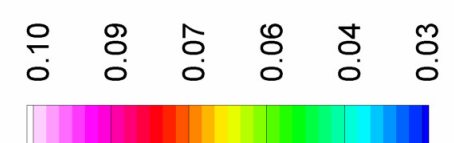

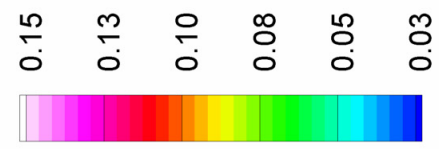
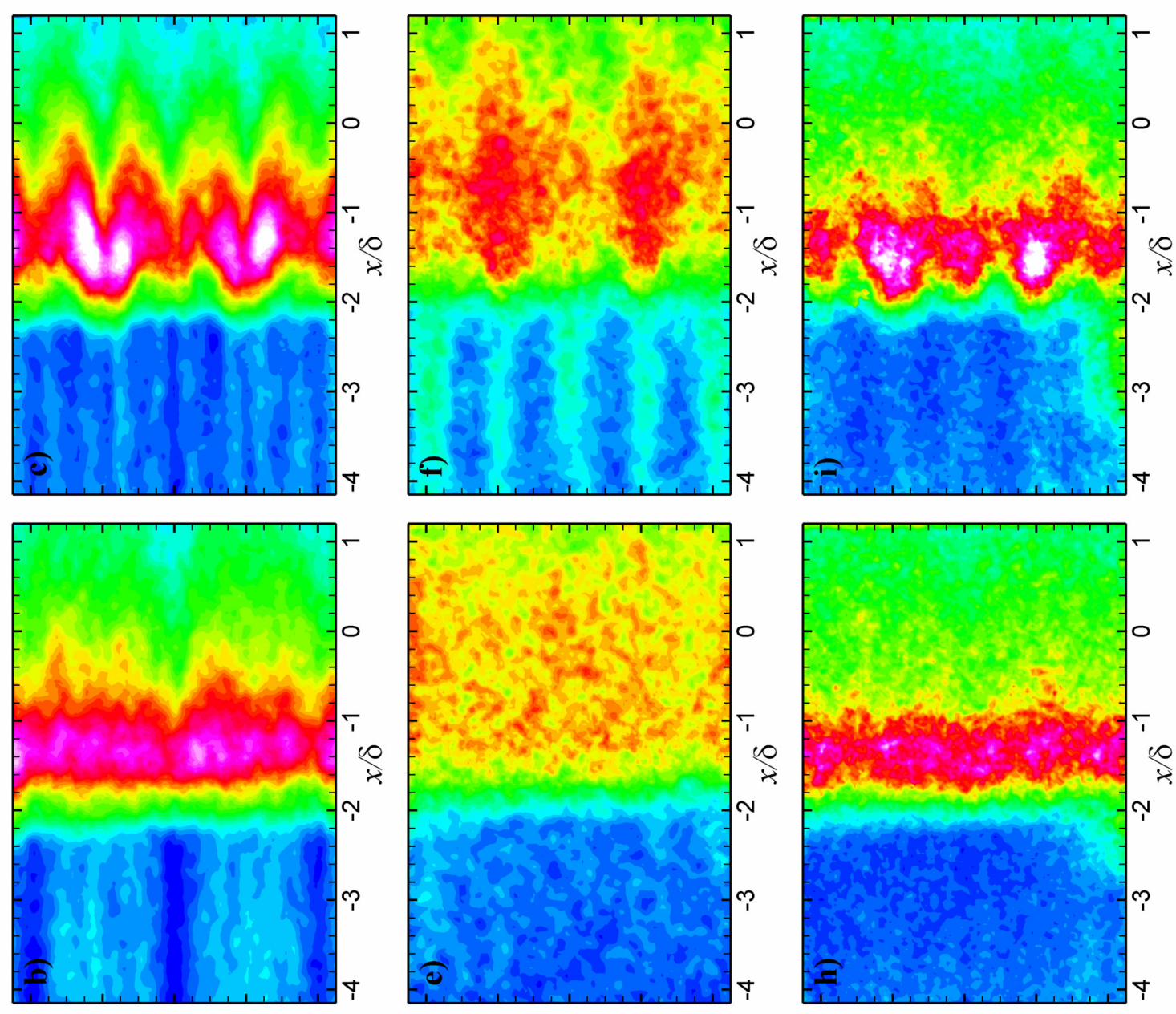

ํㅠㄹ
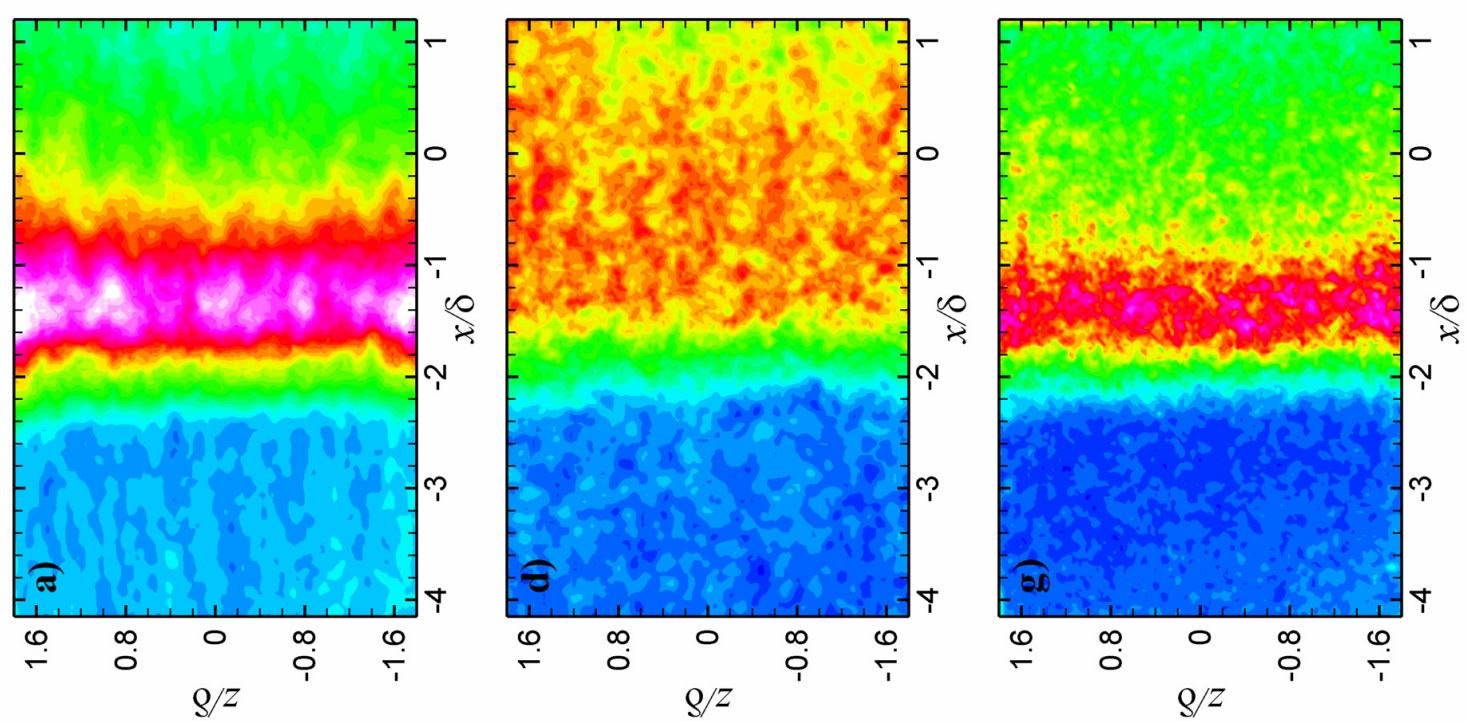

约

高高

吾

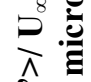

$\hat{\vec{v}} \bar{\theta}$

츨

을

宅

$D^{8}{ }^{2}$

$\hat{\mathrm{v}}$

范泀

○

家

$\sum_{n}$

$\tilde{z}$

V $气$

ஜ̈

co

ส

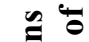

을

范

氖

更

율

늘

ํㅠㄹ

ํㅣㄹ

है

를

을 


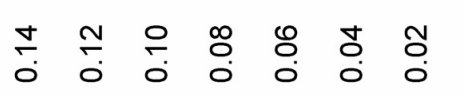
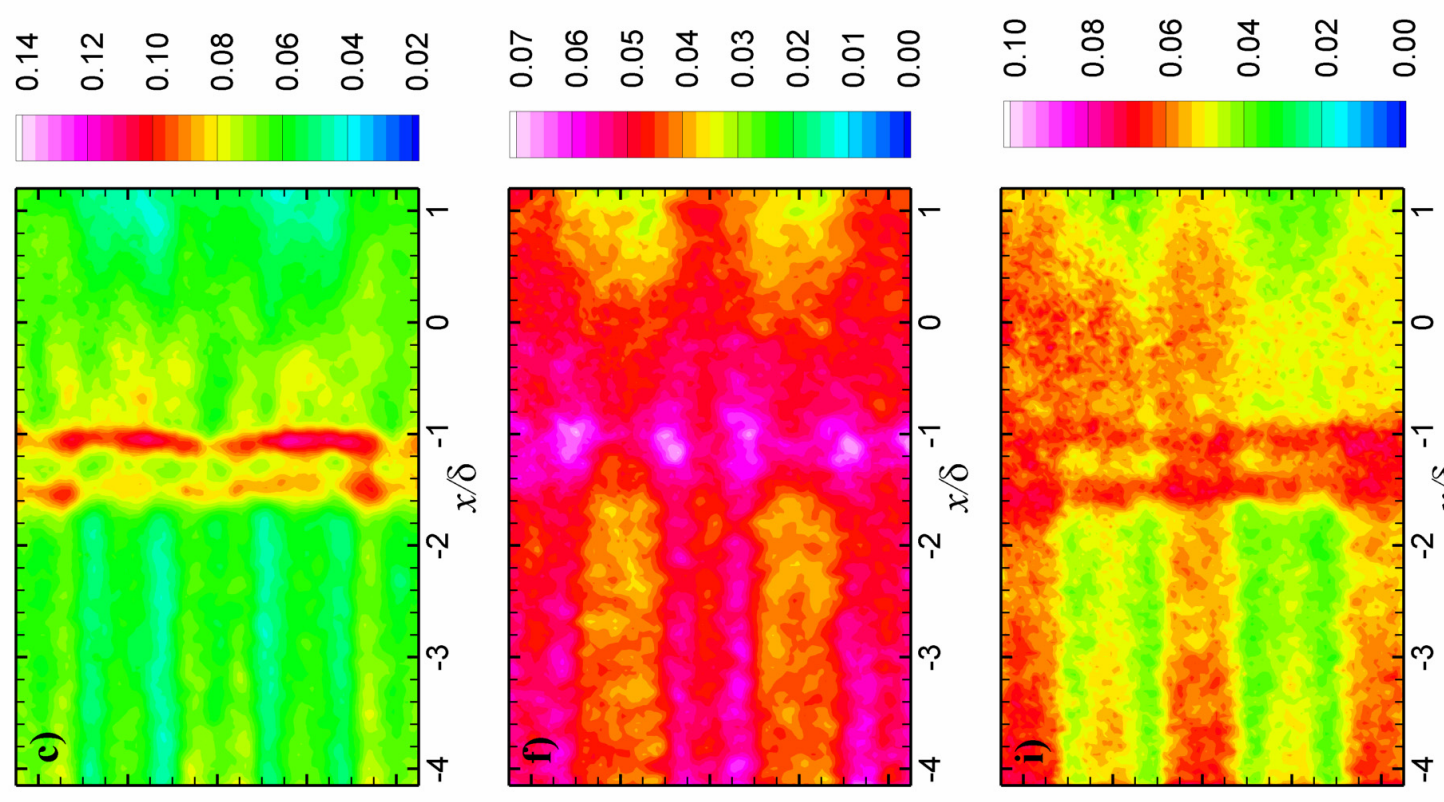

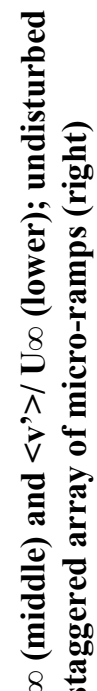
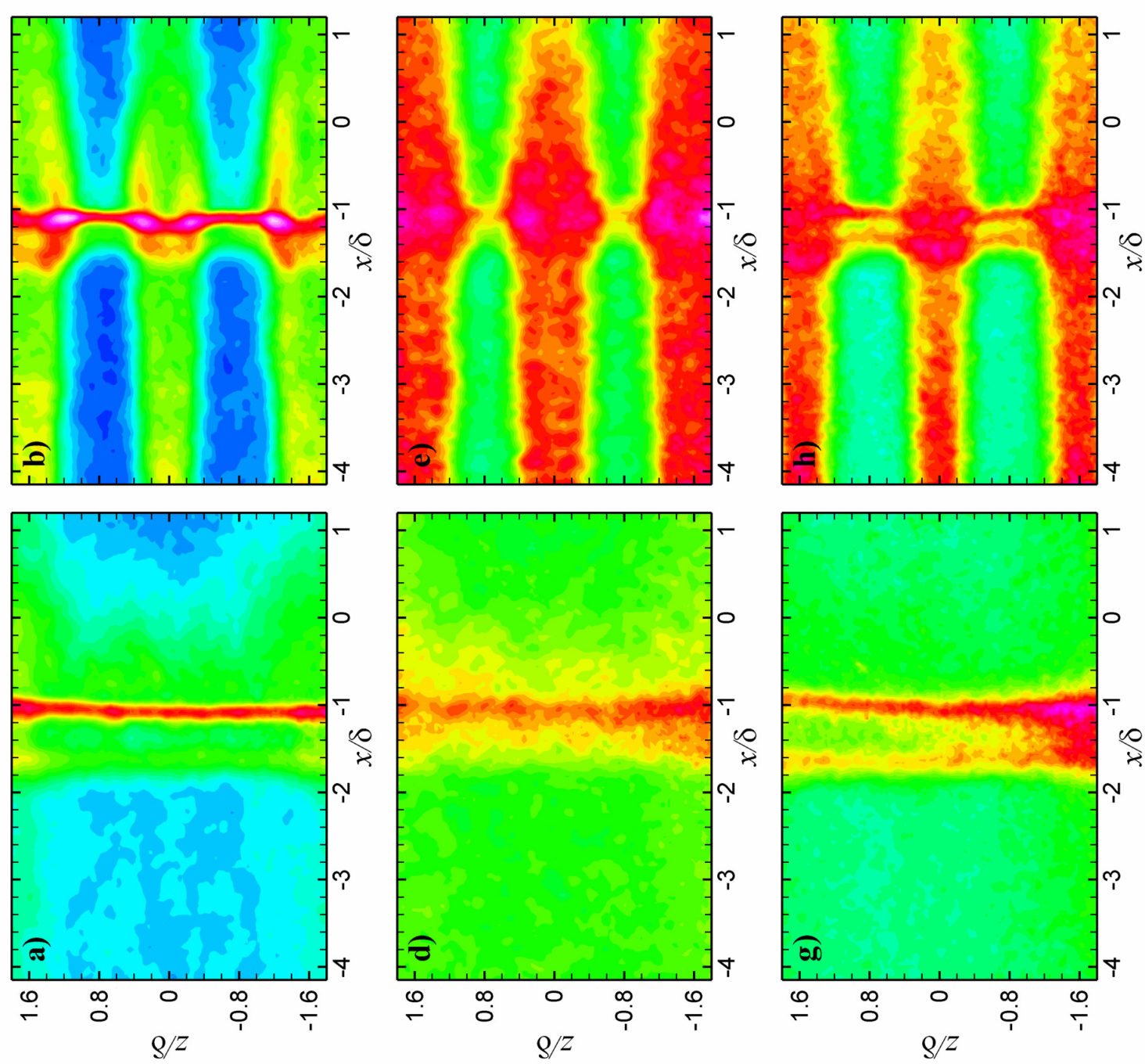

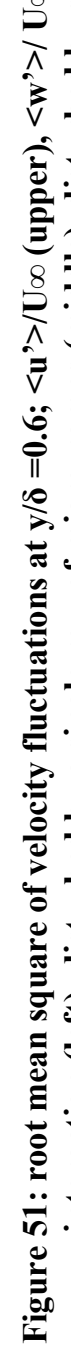




\subsubsection{Incoming boundary layer}

In order to get more insight in the effect of the micro-ramps, the turbulence levels in the incoming boundary layer are shown in figure 52. The depicted levels are the streamwise average of those from $\mathrm{x} / \delta=-4.0$ to $\mathrm{x} / \delta=-2.5$. It can be seen that the turbulence in the undisturbed boundary layer is relatively constant within the considered spanwise region.

As mentioned earlier, the effect of the single row micro-ramps on the turbulence intensity at $\mathrm{y} / \delta=0.1$ can only be discerned from $\left\langle\mathrm{u}^{\prime}\right\rangle$. The staggered array has appreciably more influence on the turbulence distribution at $\mathrm{y} / \delta=0.1:\left\langle\mathrm{v}^{\prime}\right\rangle$ and $\left\langle\mathrm{w}{ }^{\prime}\right\rangle$ are both higher downstream of the micro-ramps while $<\mathrm{u}^{\prime}>$ shows a decrease with respect to the undisturbed scenario.

At $y / \delta=0.6$, the turbulence organization in both perturbed boundary layers has significantly changed with respect to the undisturbed case. Downstream of the apices, levels are about two times as high as in the unperturbed boundary layer. Away from the apices turbulence levels have decreased compared to the unperturbed flow downstream of the single row, while in case of the staggered array no regions can be found where turbulence is lower than in the undisturbed case.

Note that in case of the staggered array, the highest levels of $\left\langle u^{\prime}>\right.$ and $<w '>$ at $y / \delta=0.6$ and of $\left\langle u^{\prime}>\right.$ at $y / \delta=0.1$ occur not exactly downstream of the vertices, but about $0.2 \delta$ next to their spanwise location. Remember that the instantaneous distance between the cores of the two counter-rotating vortices found in section 5.3.2 also is about $0.2 \delta$, suggesting that the locations of these peaks correspond to the mean locations of these cores.

Looking at $\langle\mathrm{w}$ ' $>$ it can be seen that over the entire spanwise length of the field of view, this component is larger downstream of the staggered configuration than downstream of the single-row configuration. Remember that from the instantaneous vorticity plots it was found that vorticity signatures of the two counter-rotating vortices exist along streamwise lines at vertex locations. In case of the staggered array these lines and hence these structures are located closer together relative to the characteristic size of the structures, than in case of the single row configuration. This gives ground to the speculation that the higher values for $\langle$ w' $>$ in case of the staggered array are caused by induced spanwise motions due to interaction of vortical structures. 

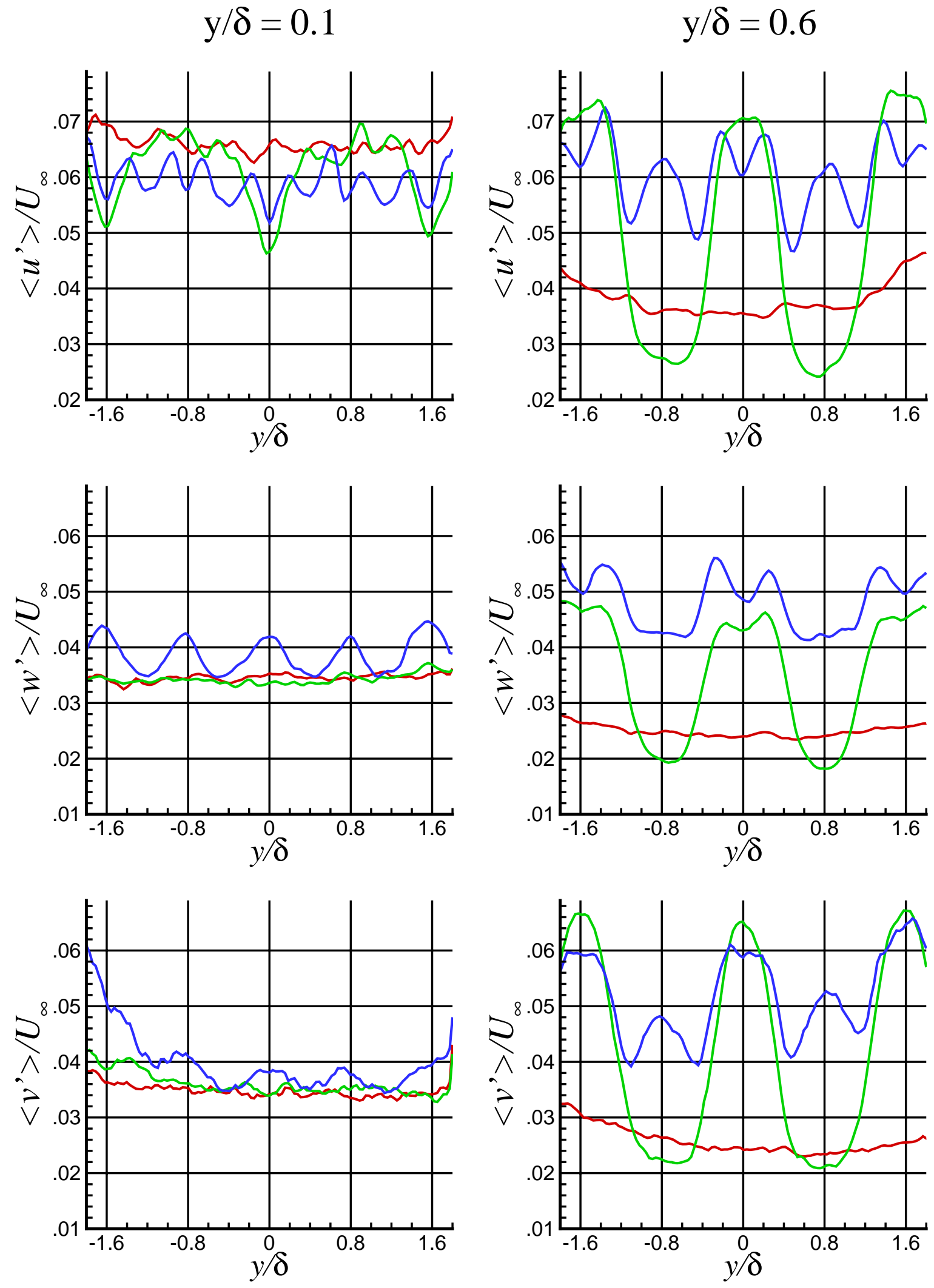

unperturbed b.1.

b.l. perturbed by a single row of micro-ramps

b.l. perturbed by a staggered array of micro-ramps

Figure 52: root mean square of velocity fluctuations in the incoming boundary layer 


\subsection{Statistical analysis}

\subsubsection{Spanwise length scales}

Autocorrelations in spanwise direction of $\mathrm{u} / \mathrm{U}_{\infty}, \mathrm{w} / \mathrm{U}_{\infty}$ and $\mathrm{v} / \mathrm{U}_{\infty}\left(R_{u u}, R_{v v}, R_{w w}\right)$ upstream and downstream of the interaction were performed in order to validate several observations from instantaneous snapshots, to further investigate the spanwise organization of the boundary layer and the effect of the impinging shock-wave thereon.

Figure 53 shows the correlations upstream of the interaction $(\mathrm{x} / \delta=-3.0)$ at a distance of $0.1 \delta$ from the wall. First the undisturbed boundary layer (the red line) will be considered. It can be seen that $R_{u u}$ drops and reaches a minimum at $0.3-0.4 \delta$ after which it rises again: a result that is in perfect agreement with the organization of the flow in longitudinal streaks with widths of $0.3-0.4 \delta$ that was found from instantaneous results. The same behaviour of $R_{u u}$ was reported by Ganapathisubramani et al. (2006). Figure 54 shows the spanwise correlations upstream of the interaction at $(\mathrm{x} / \delta=-3.0)$ at $\mathrm{y} / \delta=0.6 . R_{u u}$ can again be seen to increase after having had a relatively constant value between $0.6<|\Delta \mathrm{z} / \delta|<1.0$, supporting the organization of the boundary layer that was found from inspection of instantaneous velocity fields. This organization consists of longitudinal streaks with larger widths than those at $y / \delta=0.1$.

In order to be able to assess and compare the correlations, a representative length scale $(\lambda)$ is defined as the length for which $R=0.5$, such that $\lambda=2 \Delta \mathrm{z}_{\mathrm{R}=0.5}$; an approach that was also adopted by Ganapathisubramani et al. (2006). The resulting values for the representative length scales are presented in table 4 . The following values of $\lambda_{\mathrm{u}}, \lambda_{\mathrm{w}}$ and $\lambda_{\mathrm{v}}$ were found for the undisturbed boundary layer at $\mathrm{y} / \delta=0.1: 0.19 \delta, 0.21 \delta$ and $0.13 \delta$, indicating that the spanwise length scale based on $R_{v v}$ is significantly shorter than those based on $R_{u u}$ and $R_{w w}$, which is consistent with observation made from the instantaneous organization. At $y / \delta=0.6$, all representative lengths have increased with respect to those found at $\mathrm{y} / \delta=0.1$ : in case of the undisturbed boundary layer $\lambda_{\mathrm{u}}, \lambda_{\mathrm{w}}$ and $\lambda_{\mathrm{v}}$ now have values of $0.31 \delta, 0.28 \delta$ and $0.18 \delta$. This increase in spanwise length scales with distance from the wall has been reported by various other researchers (Smits and Dussauge 2006; Tomkins and Adrian 2003; Ganapathisubramani et al. 2006). Ganapathisubramani et al. (2006) observed values for $\lambda_{\mathrm{u}}$ which are 1.5 to 2 times larger as the present values, based on the same criteria, which is remarkable since they reported about the same widths of the streaks.

Now take a look at the spanwise correlation of the perturbed boundary layers at $y / \delta=0.1$ (see green and blue line in figure 53) It can be seen that the all depicted correlations of the boundary layer perturbed by the single-row of micro-ramps (the green line) look very much alike those of the undisturbed boundary layer. In case of staggered array (the blue line) $R_{u u}$, $R_{w w}$ and to a lesser extent $R_{v v}$ show a periodicity that conforms to the placing of the microramps. Figure 54 shows that at $\mathrm{y} / \delta=0.6$ periodicity can be found in $R_{u u}, R_{w w}$ and $R_{v v}$ of both perturbed boundary layers, although it is by far the most pronounced for $R_{u u}$. Again the periodicity closely follows the placing of the micro-ramps. As was seen throughout the previous results, the influence of most downstream row of micro-ramps in the staggered array $(|\Delta \mathrm{z} / \delta|=1.6$ and 3.2) is dominant over the influence of the most upstream row $(|\Delta \mathrm{z} / \delta|=0.8$ and 2.4).

The spanwise representative length $\lambda_{\mathrm{u}}$ can serve as a means of comparison of the typical streak width for the three considered boundary layers. It was determined that in case of the single row the perturbed $\lambda_{\mathrm{u}}$ has values of $0.20 \delta$ and $0.38 \delta$ at $\mathrm{y} / \delta=0.1$ and $\mathrm{y} / \delta=0.6$ 
respectively. In case of the staggered array, $\lambda_{\mathrm{u}}$ was found to have values of $0.22 \delta$ and $0.29 \delta$ at $\mathrm{y} / \delta=0.1$ and $\mathrm{y} / \delta=0.6$ respectively. Comparing this with values found for the unperturbed boundary layer $(0.19 \delta$ and $0.31 \delta)$, it can be stated that upstream of the interaction both configurations of micro-ramps do not significantly change the typical streak width at $y / \delta=$ 0.1 , but have a major effect on streak width at $\mathrm{y} / \delta=0.6$.

In order to assess the influence of the interaction, spanwise correlations downstream of the interaction $(\mathrm{x} / \delta=1.0)$ at $\mathrm{y} / \delta=0.1$ and at $\mathrm{y} / \delta=0.6$ are depicted in figure 55 and figure 56 respectively. Values for the representative length scales are presented in table 5.

Again the undisturbed interaction (the red line) will first be looked upon. A couple of differences can be seen with respect to the upstream correlations: to begin with, downstream of the interaction all representative lengths have increased: $\lambda_{\mathrm{u}}, \lambda_{\mathrm{w}}$ and $\lambda_{\mathrm{v}}$ now have values of $0.22 \delta, 0.25 \delta$ and $0.15 \delta$ at $\mathrm{y} / \delta=0.1$ and values of $0.33 \delta, 0.33 \delta$ and $0.18 \delta$ at $\mathrm{y} / \delta=0.6$. Secondly, $R_{u u}$ does not show such a pronounced drop to negative correlations as at $\mathrm{x} / \delta=-3.0$, suggesting that the organization in longitudinal streaks in the recovery region is less distinct in the sense that the width of elongated structures shows more variance. Thirdly, after an initial rapid drop, the slope $\mathrm{R}_{\mathrm{ww}}$ decreases, which is attributed to the coherent diverging motion away from the centreline that was encountered in previous results. At $y / \delta=0.6$ also the rate of descent of $\mathrm{R}_{\mathrm{vv}}$ decreases after an initial rapid drop. This could indicate a rather uniform downward motion in the recovery region.

Now look at the correlations downstream of the perturbed interactions (the green and blue lines in figure 55 and figure 56). Note that periodicity can only be found in case of $R_{u u}$; a result that is consistent with the wall-normal cross-section made in section 5.2.4, where no clear indication for streamwise vortices could be found from the depicted vectors. Furthermore in section 5.3.3 it was seen that in case of the perturbed interactions, the vorticity distribution downstream of the interaction has a more random nature than upstream of the interaction. It is therefore concluded that the organization vortical structures that was shown to be present in the perturbed incoming boundary layers, does not exist downstream of the interaction.

Note from the blue line in figure 56 that the effect of the most upstream row of micro-ramps $(|\Delta \mathrm{z} / \delta|=0.8$ and 2.4) in the staggered array, has completely disappeared downstream of the interaction at $\mathrm{y} / \delta=0.6$, which is consistent with plots of the mean streamwise velocity in section 5.2.4.

\begin{tabular}{l|c|c|c|c|c|c}
\hline \multirow{2}{*}{} & \multicolumn{3}{|c|}{$y / \delta=0.1$} & \multicolumn{3}{c}{$y / \delta=0.6$} \\
\cline { 2 - 7 } & $\lambda_{\mathrm{u}}$ & $\lambda_{\mathrm{w}}$ & $\lambda_{\mathrm{v}}$ & $\lambda_{\mathrm{u}}$ & $\lambda_{\mathrm{w}}$ & $\lambda_{\mathrm{v}}$ \\
\hline Undisturbed & 0.19 & 0.21 & 0.13 & 0.31 & 0.28 & 0.18 \\
\hline Single row & 0.20 & 0.22 & 0.13 & 0.38 & 0.30 & 0.22 \\
\hline Staggered array & 0.22 & 0.24 & 0.14 & 0.29 & 0.28 & 0.21 \\
\hline
\end{tabular}

Table 4: spanwise autocorrelations upstream of the interaction at $x / \delta=-3.0$

\begin{tabular}{l|c|c|c|c|c|c}
\hline & \multicolumn{3}{|c|}{$y / \delta=0.1$} & \multicolumn{3}{c}{$y / \delta=0.6$} \\
\cline { 2 - 7 } & $\lambda_{\mathrm{u}}$ & $\lambda_{\mathrm{w}}$ & $\lambda_{\mathrm{v}}$ & $\lambda_{\mathrm{u}}$ & $\lambda_{\mathrm{w}}$ & $\lambda_{\mathrm{v}}$ \\
\hline Undisturbed & 0.22 & 0.25 & 0.15 & 0.33 & 0.33 & 0.18 \\
\hline Single row & 0.22 & 0.24 & 0.15 & 0.42 & 0.29 & 0.21 \\
\hline Staggered array & 0.24 & 0.27 & 0.15 & 0.37 & 0.29 & 0.20 \\
\hline
\end{tabular}

Table 5: spanwise autocorrelations downstream of the interaction at $x / \delta=1.0$ 

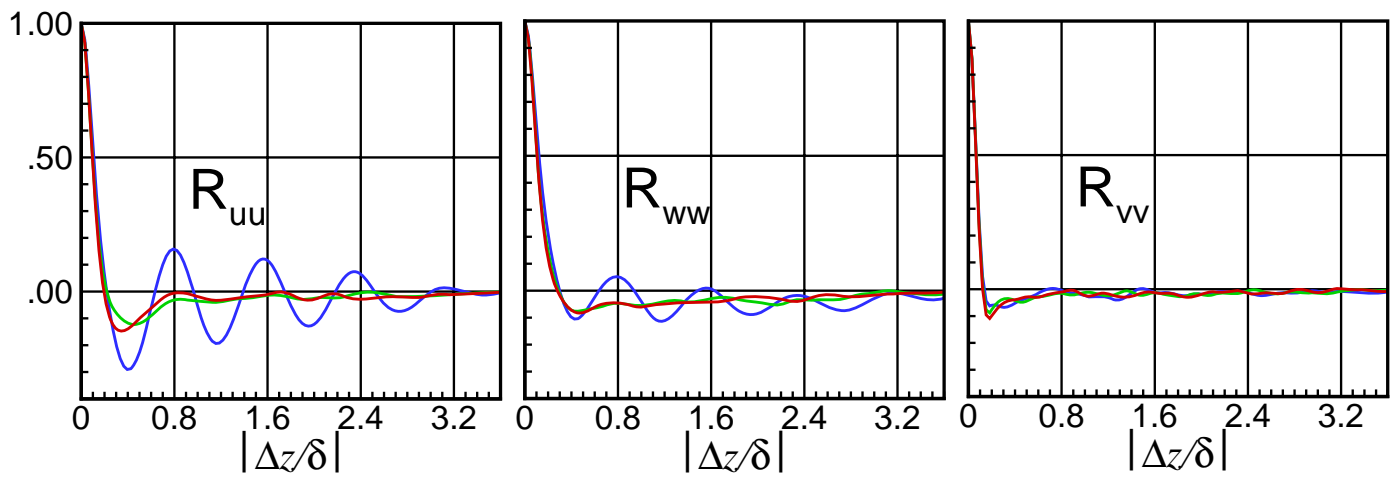

Figure 53: velocity autocorrelations at $x / \delta=-3.0, y / \delta=0.1$; the red line denotes the undisturbed case, the green line the single row case and the blue line the case of the staggered array.
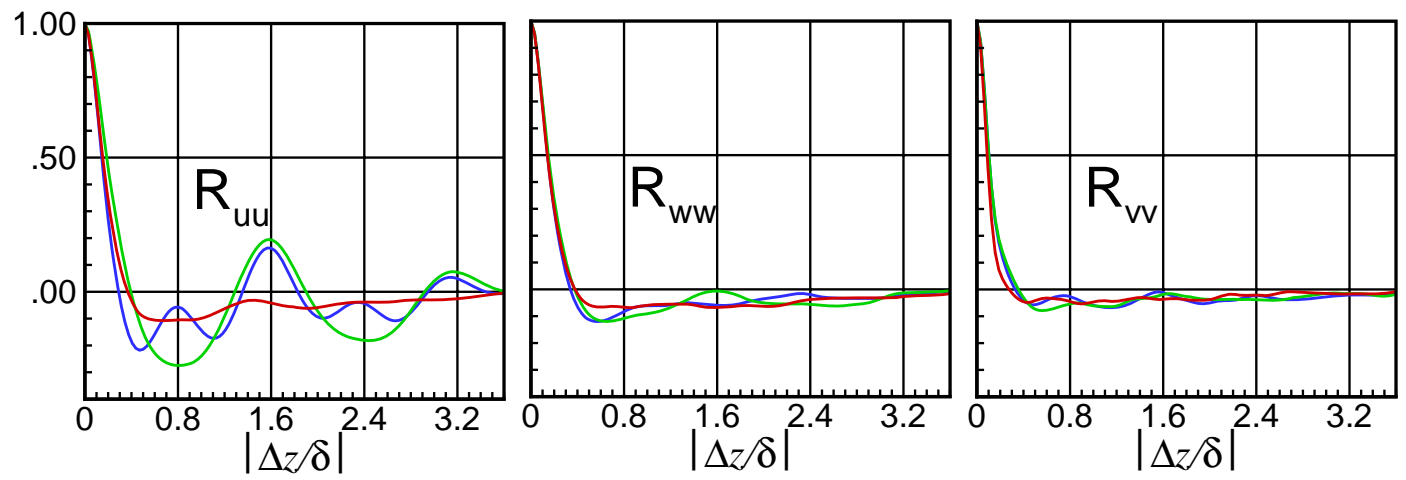

Figure 54: velocity autocorrelations at $\mathbf{x} / \delta=-3.0, y / \delta=0.6$, colour-codes as in figure 53 .
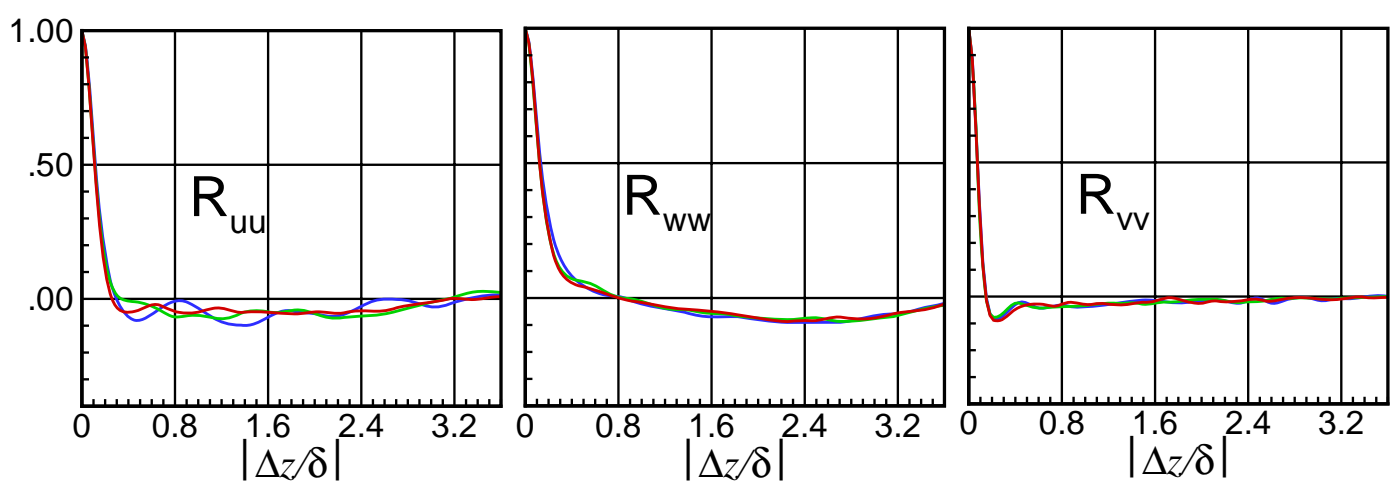

Figure 55: velocity autocorrelations at $x / \delta=1.0, y / \delta=0.1 ;$ colour-codes as in figure 53 .
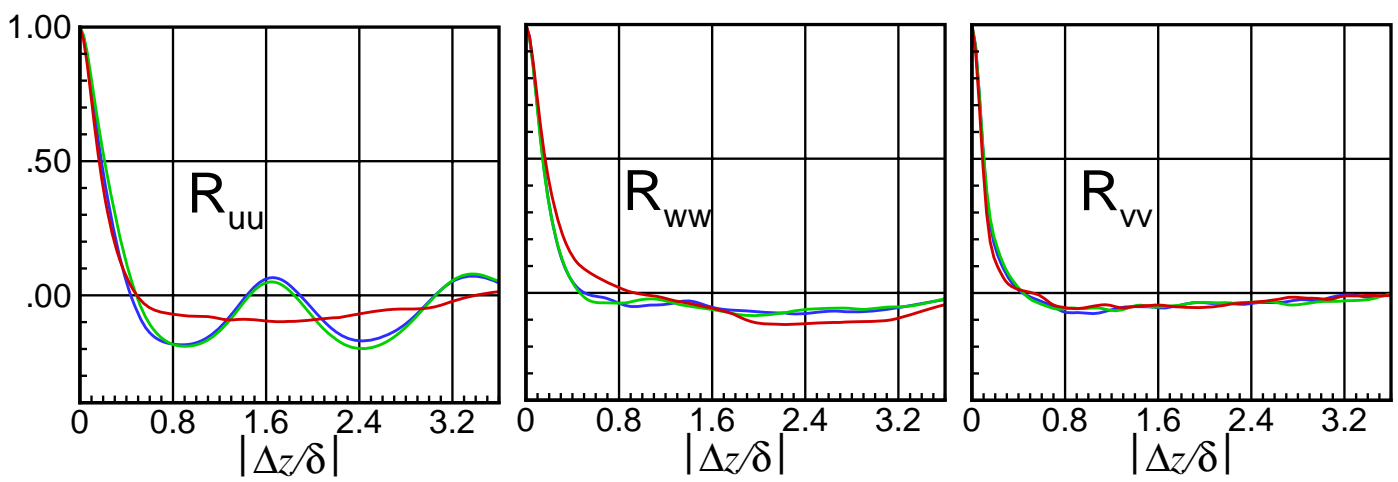

Figure 56: velocity autocorrelations at $\mathbf{x} / \delta=1.0, y / \delta=0.6$; colour-codes as in figure 53 . 


\subsubsection{Occurrence of reversed flow}

In order to assess the effectiveness of the micro-ramps in reducing separation, the percentage of the ensemble that flow reversal $\left(\mathrm{u} / \mathrm{U}_{\infty}<0\right)$ was observed at each location within the field at $\mathrm{y} / \delta=0.1$ is plotted in figure 57. The undisturbed interaction is depicted in the left figure, the interaction perturbed by a single row of micro-ramps in the middle figure and the interaction perturbed by the staggered array in the right figure. It should be noted that at $y / \delta=0.6$ no reversed flow occurs.

Figure 57a shows that the region in which flow reversal occurs in the undisturbed interaction is about $2 \delta$ long in streamwise direction. In the middle of this region, maximum flow reversal can be found in about $15 \%$ of the realizations. When micro-ramps are placed upstream of the interaction, this greatly affects the spanwise distribution of the reversed flow probability. Downstream of a micro-ramp this probability typically increases, whereas at other spanwise locations the probability can significantly decrease, which is consistent with Pitt Ford and Babinsky (2007). As a consequence, the range of maximum reversed flow probability for different spanwise positions varies between $5-20 \%$ in case of the single row configuration and between $0-40 \%$ in case of the staggered configuration as can be seen in figure $57 \mathrm{~b}$ and figure $57 \mathrm{c}$ respectively.

The figures show two features that have been seen throughout the discussion of the results: at first, the effect of the staggered array on the flow at $y / \delta=0.1$ is larger than that of the single row. Secondly, in the staggered configuration the influence of the most upstream row of micro-ramps is dominant over the influence of the most downstream row.

To further evaluate the occurrence of reversed flow, figure 58 shows the spanwise variation in the percentage of the time that flow reversal could be observed regardless of the streamwise location. Overall the figure shows the same features as were described above. Naturally, the found percentages are higher than in the former approach, where the reversed flow probability per location was considered.

The reversed flow probability of the undisturbed interaction can be seen to drop outside the range $-0.8<\mathrm{z} / \delta<0.8$ which is consistent with the observation made in the beginning of this chapter, that three-dimensional effects become significant for distance greater than $0.8 \delta$ away from the centreline. Within the range $-0.8<\mathrm{z} / \delta<0.8$ the undisturbed interaction flow reversal occurs about $30 \%$ of the time, in case of single row and the staggered array this percentage varies within the approximate ranges of $10-40 \%$ and $0-70 \%$ respectively. On average (considering $-0.8<\mathrm{z} / \delta<0.8$ ) the single row configuration and staggered configuration reduce the reversed flow probability to $24 \%$ and $21 \%$ respectively. Overall it can be concluded that flow reversal only intermittently occurs at $y / \delta=0.1$. 


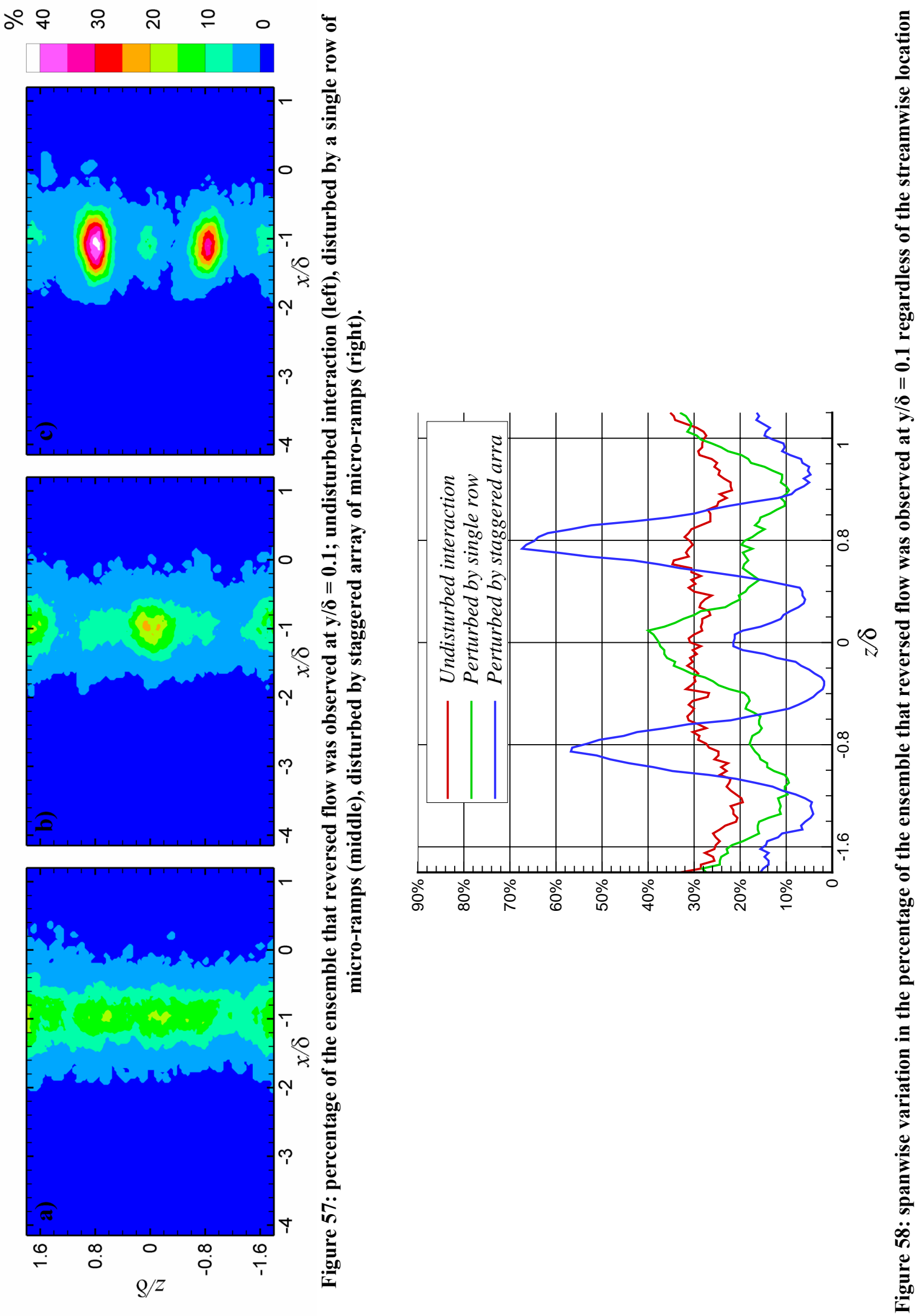




\subsubsection{Position of the reflected shock wave}

In order to gain insight into the influence of the micro-ramps on the unsteady motion of the shock wave, the relation between velocity fluctuations in the incoming boundary layer and the reflected shock wave's position is investigated for the perturbed and unperturbed interactions. The approach used is similar to that of Ganapatisubramani et al. (2007), who considered the position of a velocity isosurface as a surrogate for the streamwise position of the reflected shock wave. In case of the perturbed interactions however, the mean velocity in the incoming boundary varies with spanwise position, which would lead to an unfair comparison between different spanwise sections. Instead of a fixed velocity threshold, the following surrogate for the shock-wave's position is defined, based on the deceleration of the flow: the streamwise position where the velocity is $0.15 \mathrm{U}_{\infty}$ lower than the mean velocity at $\mathrm{x} / \delta=-3.0$. Note that this approach does not differ from that of Ganapatisubramani et al. (2007) for the undisturbed interaction, since in that case the mean velocity in the incoming boundary is uniform. It was checked that the qualitative trends of the results do not change for relative small variations in the chosen threshold $\left(-0.15 \mathrm{U}_{\infty}\right.$ W.r.t. $\left.\mathrm{x} / \delta=-3.0\right)$.

At $y / \delta=0.1$ the chosen threshold lies within the subsonic regime. Nevertheless, it is believed the chosen criterion is sufficient for the present purposes, based on the fact that the present results are in agreement with results found in literature as will be seen later in this section. The position of the surrogate position of the reflected shock, which from hereon will be referred to as the shock position, was normalized by subtracting its mean position $x_{\text {mean }}$ for a given spanwise location and dividing by the boundary layer thickness.

For each case considered, the streamwise position of the shock position was determined for all spanwise locations (126 over $3.6 \delta$ ) for 340 instances. Thereafter probability density functions (PDFs) were generated that indicate the relative probability $\left(\mathrm{N} / \mathrm{N}_{\text {tot }}\right)$ of the thresholds position falling within a certain bin, each sized $0.05 \delta$. These PDFs are shown in figure 59 . The undisturbed, single row and staggered configuration at $\mathrm{y} / \delta=0.1$ and $\mathrm{y} / \delta=0.6$ are depicted from left to right in the upper and lower row respectively. The figures suggest that the reflected shock performs a streamwise motion in the order of a boundary layer thickness and that the extent of this motion increases towards the wall, which is consistent with Dupont et al. (2008) and Humble et al. (2008). Note from the lower row, that at $y / \delta=0.6$ the mean and median positions do not coincide. Again this is consistent with Humble et al. (2008) who found the same feature of the distribution at $y / \delta=0.43$ and $y / \delta=0.82$.

The micro-ramps seem reduce the length of the intermittent region in both upstream and downstream direction. At $y / \delta=0.1$ the largest reductions typically occur downstream of the apices. This observation is supported by the fact that the standard deviation $(\sigma)$ of the shock positions (calculated for each spanwise position) attains local maxima at the corresponding spanwise positions. Looking at the lower row in figure 59, it can be seen that for the single row configuration at $\mathrm{y} / \delta=0.6$ the largest reductions in the length of the intermittent region can now be found at spanwise locations in between the micro-ramp vertices, which signifies a trend reversal with respect to $\mathrm{y} / \delta=0.1$. The influence of the staggered array shows more spanwise uniformity at $y / \delta=0.6$. These observations are again supported by the spanwise location of local maxima in the standard deviation of the shock positions. Remember that a trend reversal with distance from the wall was also found in spanwise variation in the turbulence intensity in streamwise direction $\left\langle u^{\prime}\right\rangle$, suggesting that the shock motion is related to fluctuations in the streamwise velocity as was also hinted by instantaneous velocity fields. 


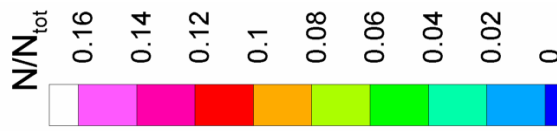
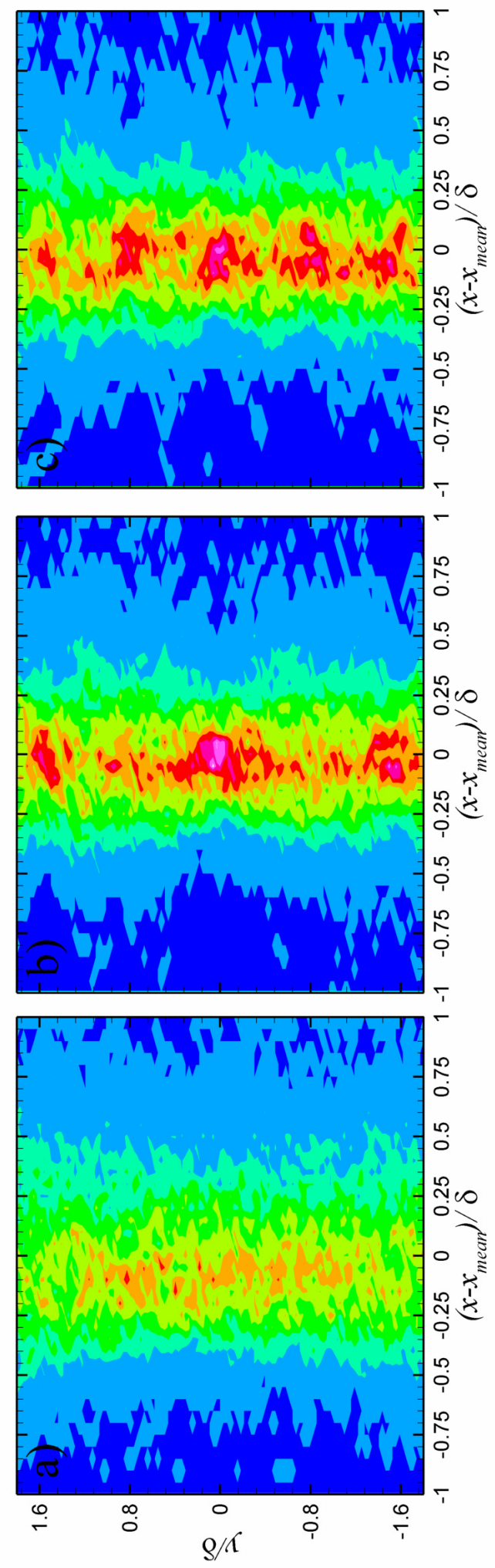

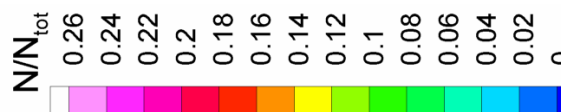
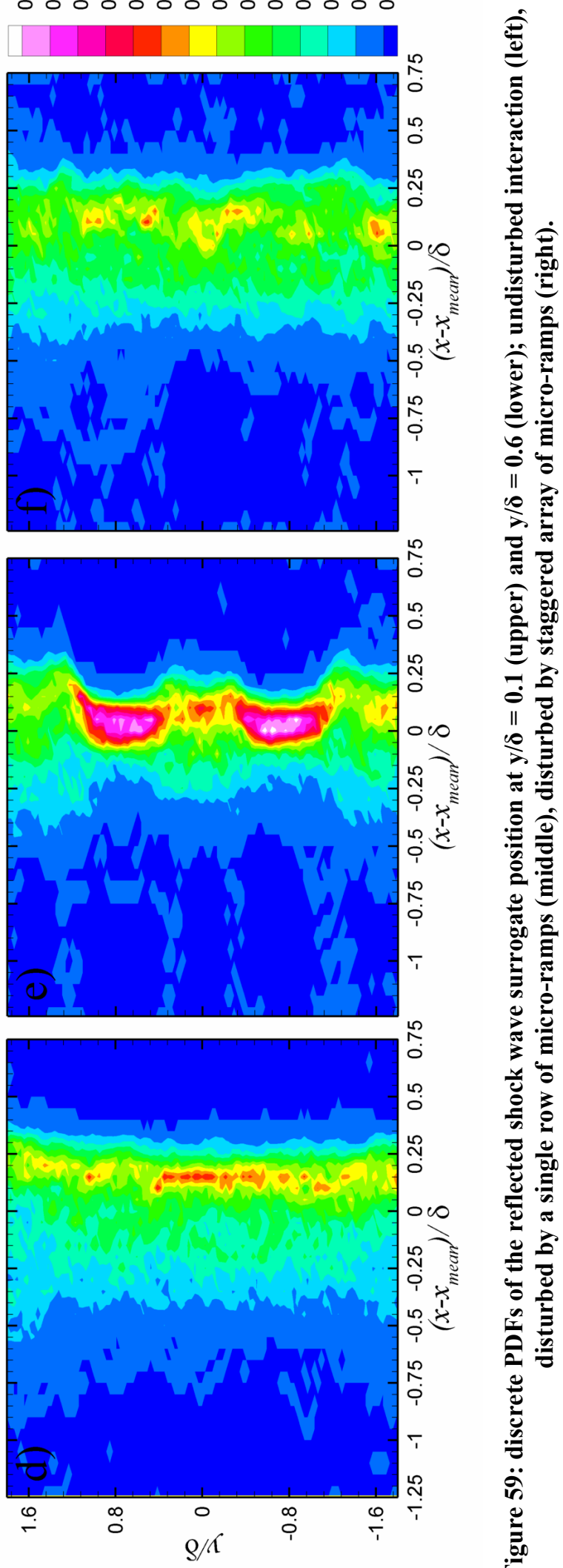

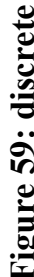


In order to assess the spanwise average effect of the micro-ramps on the length of the intermittent region, the discrete PDFs averaged over $-0.8<\mathrm{z} / \delta<0.8$ are shown in figure 60 . Like before, the undisturbed, single row and staggered configuration at $\mathrm{y} / \delta=0.1$ and $\mathrm{y} / \delta=0.6$ are depicted from left to right in the upper and lower row respectively. It can be seen that at $\mathrm{y} / \delta=0.1$ the spanwise averaged reduction in intermittent region is similar for both configurations as they both decrease the standard deviation of the shock positions by about $20 \%$ with respect to that of the undisturbed interaction. At $\mathrm{y} / \delta=0.6$ the single row configuration on average reduces the standard deviation of the shock positions with $30 \%$ with respect to that of the undisturbed interaction, which indicates a significant reduction in the extent of the shock motion. The staggered configuration at $y / \delta=0.6$ on averaged does not affect the standard deviation of the shock positions but does seem to reorganize the shock's motion in the sense that the shape of the discrete PDF now more resembles a normal distribution than that of the undisturbed interaction.
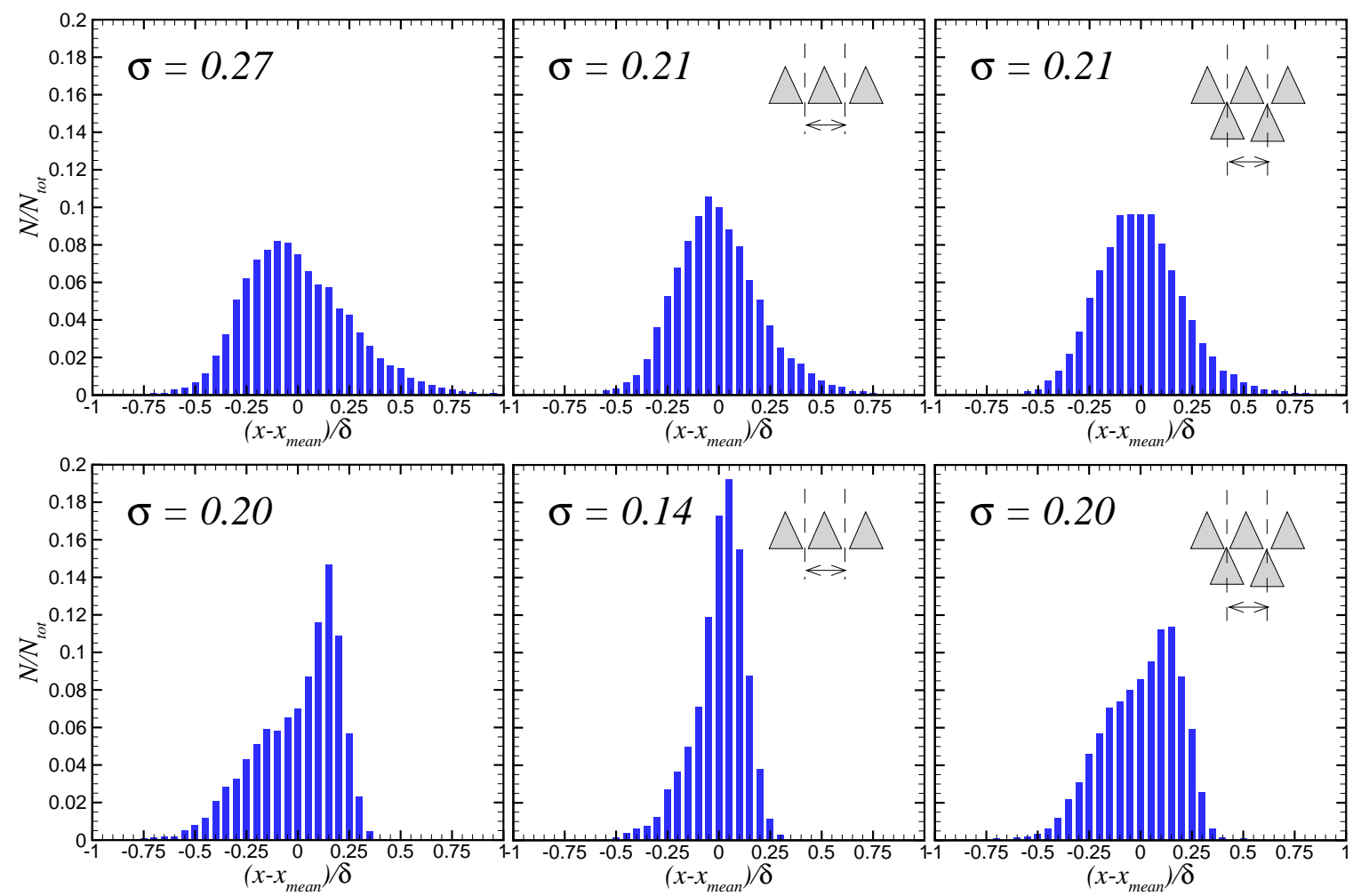

Figure 60: discrete PDFs of the reflected shock wave surrogate position averaged over $-0.8<z / \delta<0.8$ at $y / \delta$ $=0.1$ (upper) and $y / \delta=0.6$ (lower); undisturbed interaction (left), disturbed by a single row of microramps (middle), disturbed by staggered array of micro-ramps (right); $\sigma$ denotes the spanwise averages standard deviation of the shock positions.

\subsubsection{Relation between velocity fluctuations and shock position}

In order to gain more insight in the dynamics of the interactions, the relation between the velocity fluctuations in the field of view and the shock wave's position will statistically be investigated. In figure 61 , the distribution of streamwise velocity fluctuations $\mathrm{u}^{\prime} / \mathrm{U}_{\infty}$ in the incoming boundary layer at $\mathrm{x} / \delta=-2.7$ and the reflected shock position $\left(x-x_{\text {mean }}\right) / \delta$ are shown on the left and joint PDFs of the two variables are shown on the right. The upper figures correspond with $\mathrm{y} / \delta=0.1$ and lower figures with $\mathrm{y} / \delta=0.6$. 
It can be deduced from the figures that the both the range of shock positions as the range of velocities at $\mathrm{y} / \delta=0.1$ are larger than at $\mathrm{y} / \delta=0.6$; a result that was seen earlier in the discussion of the instantaneous flow organization and that is consistent with Ganapathisubramani et al. (2007) and Humble et al. (2008). In addition, it can clearly be seen that the contours of the joint PDF are epileptically in the sense that they indicated that when a positive velocity fluctuation is present in the incoming boundary layer the shock is more likely to be located downstream of its median position and vice versa. This result is in agreement previous observations, with joint PDFs found by Ganapathisubramani et al. (2007) and Humble et al (2008), and with results obtained by Beresh et al. (2002).

Correlation coefficients corresponding to the plots at $\mathrm{y} / \delta=0.1$ and $\mathrm{y} / \delta=0.6$ are 0.32 and 0.20 respectively, indicating this relation is stronger closer to the wall. Again this is consistent with previous observations and results reported by Beresh et al. (2002) and Humble et al. (2008).

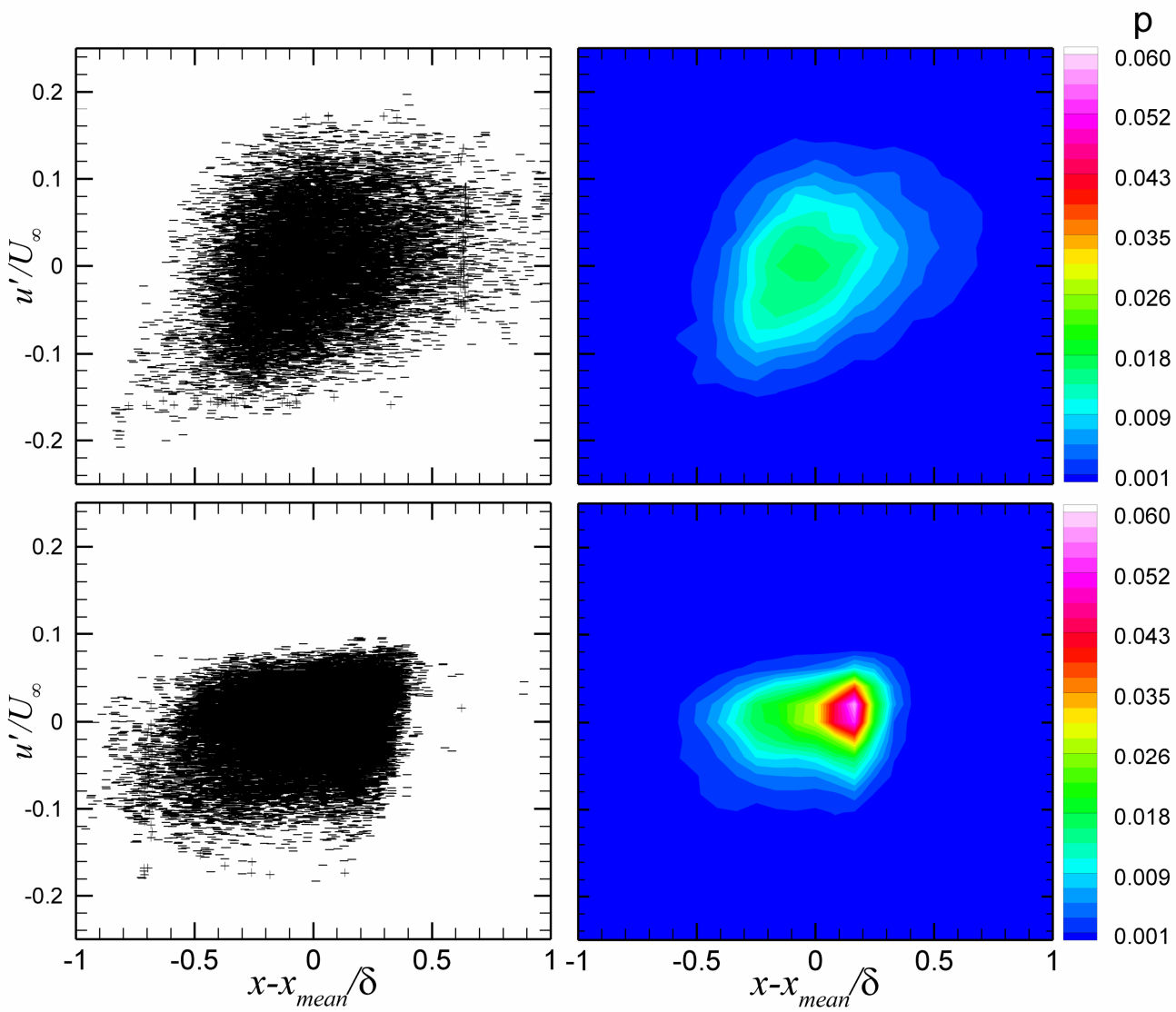

Figure 61: relationship between streamwise velocity fluctuations $u^{\prime} / U_{\infty}$ at $x / \delta=-2.7$ and the reflected shock surrogate position $\left(x-x_{\text {mean }}\right) / \delta$; distributions of these two variables are shown on the left and joint PDFs are shown on the right; upper figures correspond with $y / \delta=0.1$ and lower figures with $y / \delta=0.6$; correlation coefficient are 0.33 and 0.20 respectively.

In order to visualize the relation between the shock position and the velocity fluctuations at a given streamwise position, the correlation coefficient between these two variables are plotted in figure 62 and figure 63 for $y / \delta=0.1$ and $y / \delta=0.6$ respectively. It can be seen from figure 62 that at $\mathrm{y} / \delta=0.1$ the correlation decreases away from the mean shock wave position. The rate of descent of the correlation becomes smaller at approximately the borders of the intermittent region, which were found in the previous section. The correlation at these borders is about 0.3 . For unknown reasons this value is significantly smaller than that found by Ganapathisubramani et al. (2007) and Humble et al. (2008), who reported correlations 
coefficients of 0.4 and 0.5 respectively between the flow in the incoming boundary layer and the shocks position.

Figure 63 shows that at $y / \delta=0.6$ the correlation peak is not as broad as at $y / \delta=0.1$. At streamwise positions lay outside the intermittent region the correlation coefficient is about 0.2 in case of the undisturbed interaction.

Note that at $y / \delta=0.1$, the plots are quasi-symmetrical which suggests that both downstream and upstream velocity fluctuations are equally correlated to the shock's position. At $y / \delta=0.6$, the drop in the correlation away from the correlation peak conforms to the probability density function of the shocks position (see previous section) in the sense that the drop in probability it is also more spread out in upstream than in downstream direction. Outside the intermittent region the correlation with the shocks position is similar for the both downstream and upstream velocity fluctuations, just like at $\mathrm{y} / \delta=0.1$.

It can be seen from both figures that the correlation of the perturbed flow with the shocks position is to typically less than that of the undisturbed flow. It is speculated that this can be attributed the smaller shock motions in streamwise direction downstream of the micro-ramps which was found in the previous section. Another reason for the reduced correlation could be that low-speed streaks downstream of the micro-ramps at $\mathrm{y} / \delta=0.6$ consist of multiple structures rather than being an elongated streak with uniform velocity. 


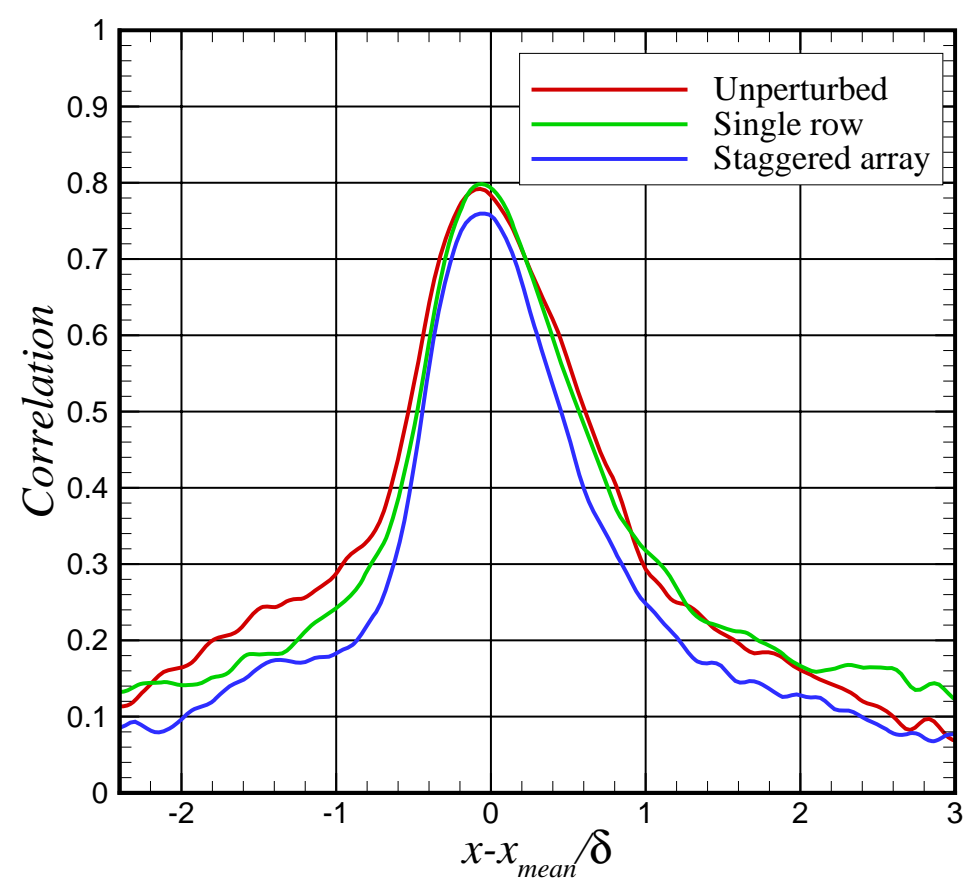

Figure 62: correlation between streamwise velocity fluctuations $u^{\prime} / U_{\infty}$ at $\left(x-x_{\text {mean }}\right) / \delta$ and the location of the reflected shock wave surrogate position; at $y / \delta=0.1$.

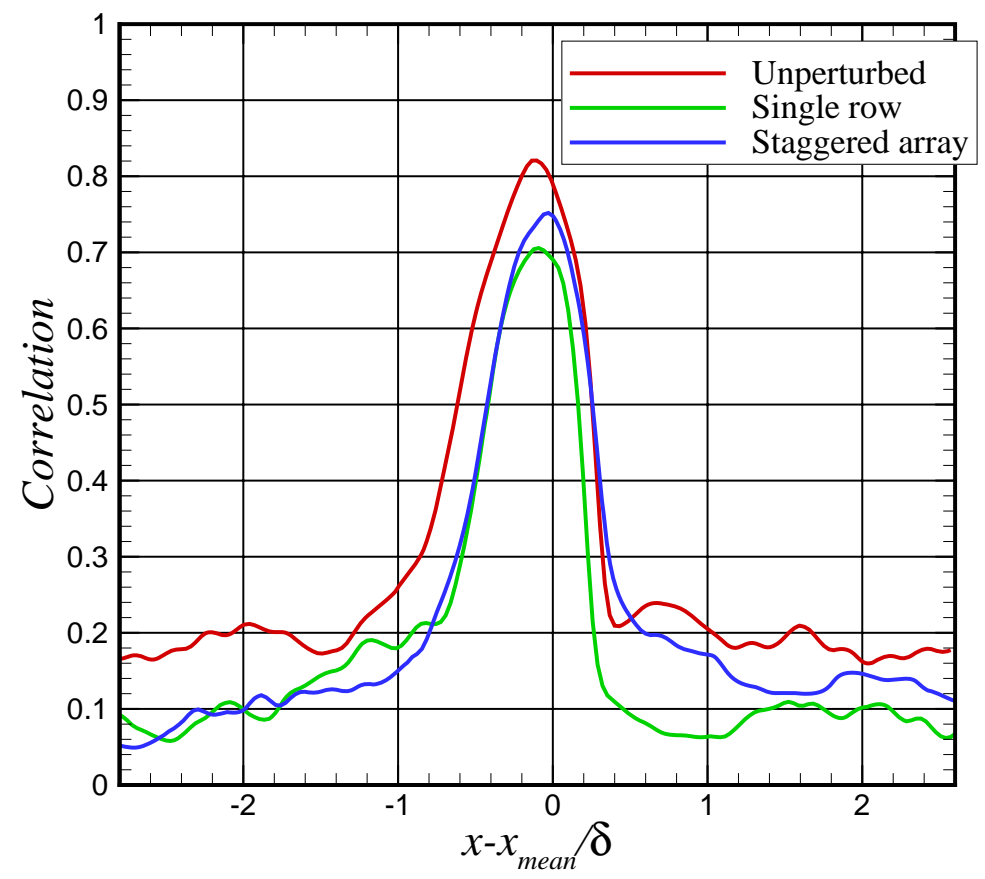

Figure 63: correlation between streamwise velocity fluctuations $u^{\prime} / \mathbf{U}_{\infty}$ at $\left(x-x_{\text {mean }}\right) / \delta$ and the location of the reflected shock wave surrogate position; at $y / \delta=0.6$. 



\section{Chapter 6: Conceptual Model}

We now consolidate the major observations in this thesis into a plausible conceptual model of the effects of micro-ramps on the SWTBLI. We first describe the effects of the micro-ramps on the incoming boundary, followed by the interaction itself.

\subsection{Incoming boundary layer}

The instantaneous structure of the undisturbed boundary consists of alternating coherent streamwise streaks of low- and high-speed velocity, although this structure is more common at $0.1 \delta$ than at $0.6 \delta$ away from the surface. Vortical structures are present at the interfaces of these streaks. At greater distances from the wall the characteristic width of the streaks increases together with all other characteristic lengths in spanwise direction, while the velocity difference between low- and high-speed streaks decreases. Overall, these results are consistent with the hairpin-packet model of incompressible turbulent boundary layers (Kim and Adrian, 1999; Tomkins and Adrian 2003).

When micro-ramps are placed in the boundary layer, the instantaneous structure of the layer close to the wall remains unchanged $(0.1 \delta)$. However, where in the unperturbed layer the streaks are randomly distributed, their locations are to some extent determined by the spanwise positions of micro-ramps: downstream of vertex locations low-speed streaks are more likely to occur. The opposite is true for spanwise positions away from the vertices.

Father away from the wall $(0.6 \delta)$ the instantaneous structure of the boundary layer changes significantly. The locations of high- and low-speed regions are now almost completely determined by the spanwise placing of the micro-ramps. Additionally, low-speed regions downstream of vertex locations are no longer coherent in streamwise direction, like in the unperturbed boundary layer, but contain individual structures that pass by at regular intervals of about a boundary layer thickness, leading to an increase in turbulence intensity.

These structures consist of pairs of counter-rotating vortices in a wall-parallel plane that also appear to have a streamwise vorticity component and therefore have features in common with hairpin vortices. High-speed regions are located at intermediate spanwise locations. A schematic of this instantaneous organization drawn over an instantaneous vorticity field is shown in figure 64. It should be emphasized that the existence of hairpin-like structures, as drawn in this figure, is subject of tentative speculation. 


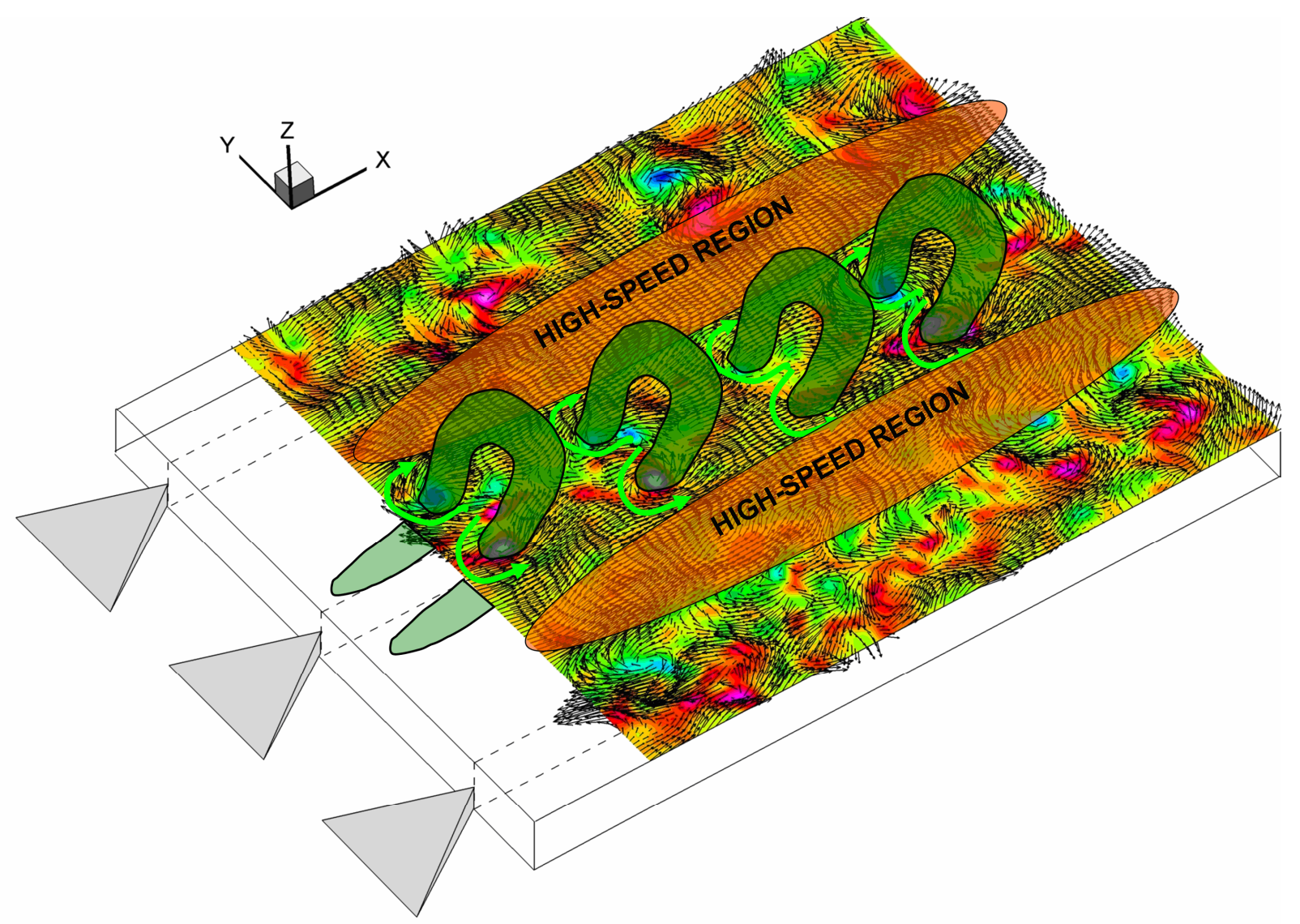

Figure 64: instantaneous vorticity field of the incoming boundary layer with a drawn in conceptual model that incorporates the presence of hairpin vortices inclined to the surface downstream of vertex locations (transparent green) and high-speed regions at intermediate spanwise positions (transparent orange); vectors are shown in a convective reference frame of the local mean streamwise velocity $\left(0.73 U_{\infty}\right)$; the micro-ramps as well as their distance from the field of view are not drawn on scale; height of the hairpin vortices was chosen arbitrarily.

The lowest mean velocities occur downstream of vertex locations and the highest at intermediate spanwise locations. Additionally, mean results put in evidence pairs counterrotating vortices downstream vertex locations in the cross-flow plane, such that in the region between them the induced flow is away from the surface as is illustrated in

figure 65, which shows a conceptual sketch of the mean effect of the micro-ramps on the perturbed incoming boundary layer and SWTBLI. These results together with results from literature (e.g. Holden and Babinsky 2007; Pitt Ford and Babinsky 2007) suggest that longitudinal streamwise vortices are responsible for the distribution of mean streamwise velocity as they induce a mixing of the boundary layer by moving low-velocity fluid away from the wall downstream of the micro-ramp's vertices and high-velocity fluid towards the wall at intermediate spanwise positions. Present instantaneous results however showed that the mean velocity distribution is a time-averaged effect of individual vortex pair packets and not of steady vortical structures.

The staggered configuration of micro-ramps has a greater effect on the flow close to the wall $(0.1 \delta)$ than the single-row configuration. The strength of the effect of micro-ramps is thus, amongst other factors, dependent on the spacing between the pairs of vortices. Based on oil- 
flow visualizations and results from literature, this dependency has been related to migration of vortices away from the wall.

In case of the staggered configuration the most downstream row of micro-ramps has a dominant influence on the flow organization at $0.6 \delta$ away from the wall, while closer to the wall $(0.1 \delta)$ the most upstream row is largest. It was suggested that the most downstream row of micro-ramps creates a downward flow that moves the flow coming from the most upstream ramps closer to the wall.

\subsection{SWTBLI}

When a shock impinges on the boundary layer, the flow starts to decelerate upstream of the location where the shock would impinge on the wall in absence of a boundary layer. This deceleration is accompanied by an increase in turbulence intensity of all three components and in wall-normal vorticity. Downstream of the interaction the fluid accelerates, while turbulence and vorticity levels relax again. In the recovery region spanwise characteristic lengths are larger than in the incoming boundary layer.

Although on the mean this process is relatively two-dimensional, instantaneously significant variations are present due to low- and high-speed streaks in the incoming boundary layer which convect through the interaction. Downstream of a low-speed streak the position of reflected shock, based on a velocity contour, is typically located more upstream, the minimum velocity that is attained in the interaction is lower and the recovery of the flow downstream the interaction slower. The opposite is true downstream of a high-speed streak and the instantaneous shape of the subsonic region therefore usually conforms to the velocity distribution in the incoming boundary layer.

Father away from the wall $(0.6 \delta)$ the spanwise organization of the interaction is less dependent on the presence of low- and high-speed regions in the incoming boundary layer and the streamwise motion of the reflected shock is smaller than closer to the wall $(0.1 \delta)$.

When a shock impinges on boundary layer perturbed by micro-ramps, the interaction is no longer two-dimensional as in the undisturbed interaction. The low- and high-speed regions in the perturbed incoming boundary layer have the same effect as the instantaneous streaks in the unperturbed incoming boundary layer: downstream of vertex locations where the mean velocity in the incoming boundary layer is lowest, the mean position of reflected shock, based on a velocity contour, is located more upstream, the minimum mean velocity that is attained in the interaction is lower, the mean recovery of the flow downstream the interaction slower and vice versa. The mean shape of the subsonic region therefore conforms to the mean velocity distribution in the incoming boundary layer, thereby deflecting the flow in both wallnormal and spanwise direction as shown in

figure 65 .

The spanwise averaged effect of the micro-ramps, although weak, is the same as in the incoming boundary layer: close to the wall $(0.1 \delta)$ the mean velocity increases with respect to unperturbed case, while farther away from the wall $(0.6 \delta)$ it decreases.

The probability of separation which is uniform in spanwise direction in case of the undisturbed interaction shows significant spanwise variation in case of the perturbed interactions, being highest downstream of vertex locations and lower at intermediate spanwise 
positions. The spanwise averaged probability of separation becomes lower than that in the undisturbed interaction.

Overall the streamwise movement of the reflected shock decreases downstream of the microramps in both upstream and downstream direction, although there is a spanwise variation in this reduction. Close to the wall, the largest reductions occur downstream of the vertices of the micro-ramps, while farther away from the wall the reductions are strongest away from the vertices. This trend reversal follows the trend reversal of the effect of the micro-ramps on the streamwise component of the RMS velocity in the incoming boundary layer.

The interaction has a detrimental effect on the instantaneous and mean structure of incoming the boundary layer. Downstream of the interaction the instantaneous vorticity is more randomly distributed with respect to that in the incoming boundary layer. As a result, the mean structure of streamwise vortices is largely lost as well, although the spanwise variation in streamwise velocity induced by the micro-ramps is still partially present.

Like in the incoming boundary layer, the staggered configuration of micro-ramps has a greater effect on the flow close to the wall $(0.1 \delta)$ than the single-row configuration. Also, in case of the staggered configuration the most downstream row of micro-ramps has a dominant influence on the flow organization $0.6 \delta$ away from the wall, while closer to the wall $(0.1 \delta)$ the most upstream row is largest. For speculations about the causes of these observations the reader is referred to the previous section on the incoming boundary layer.

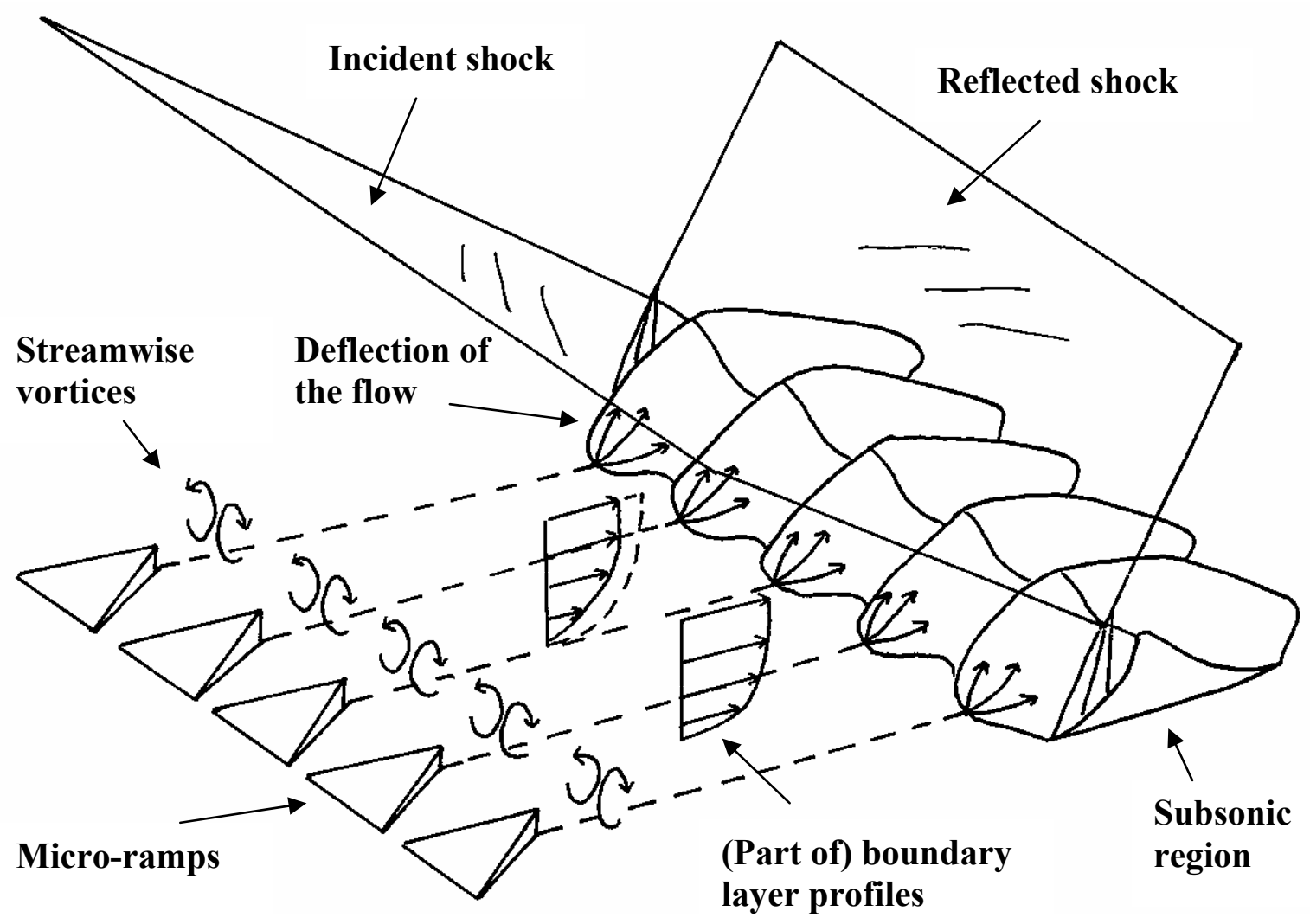

Figure 65: conceptual sketch of the perturbed interactions; drawing is not on scale; depicted parts of boundary layer profiles are meant to illustrate spanwise variation of velocity and should not be regarded as an attempt to estimate their size and shape 


\section{Chapter 7: Conclusions and Recommendations}

\subsection{Conclusions}

Stereo-PIV measurements in wall-parallel planes at $0.1 \delta$ and $0.6 \delta$ away from away from a flat plate surface have been performed to investigate the effect of two configurations of microramps, a single row and a staggered array, on an oblique shock reflection at a freestream Mach number of 1.84. Additionally, oil-flow patterns at the surface were obtained. Results on the mean and instantaneous flow as well as the turbulence statistics distribution for both the perturbed and unperturbed cases were obtained and discussed. Additionally, a statistical analysis was performed to further investigate the effects of micro-ramps on the flow structure in terms of spanwise length scales, reversed flow probability and shock motion. In order to consolidate the major observations, a conceptual model was formulated of the boundary layer's and interaction's topology and the effect of micro-ramps thereon. As a result of this work, several conclusions may be drawn:

PIV is a well-suited flow diagnostic technique in the investigation the effects of micro-ramps on SWTBLIs. The micro-ramps generate individual vortex pair packets downstream of their vertices that on the mean look like longitudinal streamwise vortex pairs. These structures perturb the incoming boundary layer such that on the mean low-speed regions exist downstream of the vertices and high-speed regions at intermediate locations. Downstream of the low-speed regions in the incoming boundary layer, the probability of reversed flow occurrence in the interaction region is higher, and the subsonic region is longer in streamwise direction. Overall, the probability of the occurrence of reversed flow in the interaction region at $0.1 \delta$ away from the surface decreased by $20 \%$ and $30 \%$ downstream of the single row and the staggered configuration respectively. Both configurations of micro-ramps stabilize the shock motion by reducing the length of its motion by about $20 \%$ at a distance of $0.1 \delta$ from the surface. Farther away from the wall $(0.6 \delta)$ only the single row configuration was found to be effective, leading to a spanwise averaged reduction of $30 \%$.

\subsection{Recommendations}

Since it was shown that micro-ramp control is effective in stabilizing the interaction, it offers promising prospects for the control of SWTBLIs. This is particularly true in case of engine applications for which robustness is a warranted characteristic of control devices. It is therefore recommended to further develop micro-ramp control, whereby the conceptual model proposed in the present investigation can provide (part of) an initial framework in which this can be done. The author regards research on the effect of on the shape and size of microramps as well as their spacing with respect to the interaction and each other, as a logical next step in the process of developing micro-ramp control. Also research on the effect of microramp control on structural and thermal loads will become indispensable to assist trade-offs in practical design. Additionally, it is envisaged that research on interaction control in general will help to gain more insight in the dynamics of the uncontrolled interaction. 
EFFECTS OF MICRO-RAMPS ON A SHOCK WAVE BOUNDARY LAYER INTERACTION 


\section{References}

Adamson, T.C. Jr. and Messiter, A.F., Analysis of two-dimensional interactions between shock waves and boundary layers," Annu. Rev. Fluid Mech. 12, pp. 103-138, 1980.

Anderson, B.H., Tineapple, J. and Surber, L., "Optimal Control of Shock Wave Turbulent Boundary Layer Interactions Using Micro-Array Actuation," 3rd AIAA Flow Control Conference, AIAA 2006-3197, San Francisco, CA, June 2006.

Ashill, P.R., Fulker, J.L. and Hacket, K.C., "Research at DERA on Sub Boundary Layer Vortex Generators (SBVGs)", $39^{\text {th }}$ AIAA Aerospace Sciences Meeting and Exhibit Jan, Reno, Nevada, AIAA 2001-0887, Jan. 2001.

Barter, J.W. and Dolling, D.S., "Reduction of Fluctuating Pressure Loads in Shock/Boundary-Layer Interactions Using Vortex", AAIA Journal, Vol. 33, No. 10, pp. 1842-1849, Oct. 1995.

Beresh S.J., Clemens, N.T. and Dolling, D.S., "Relationship Between Upstream Turbulent Boundary Layer Velocity Fluctuations and Separated Shock Unsteadiness", AIAA Journal, Vol. 40, No 12, pp 2412-2422, Dec. 2002.

Bueno, P.C., Wagner, J.L., Searcy, J.A., Ganapathisubramani, B., Clemens, N.T. and Dolling, D.S., "Experiments in Unsteady Forcing of Mach 2 Shock Wave/Boundary Layer Interactions", 44 ${ }^{\text {th }}$ AIAA Aerospace Sciences Meeting and Exhibit Jan, AIAA 2006-878, Reno, Nevada, Jan. 2006.

Bur, R., Benay, R., and Délery, J.M., "Physical Study of Shock-Wave/BoundaryLayer Interaction Control in Transonic Flow", $38^{\text {th }}$ Aerospace Sciences Meeting \& Exhibit, Reno, NV, AIAA 2000-0933, Jan. 2000.

Délery, J.M., "Shock/Wave/Turbulent Boundary Layer Interaction and its Control", In: Prog.Aerospace.Sci., Vol. 22, pp. 209-280, Pergamon Press Ltd., Great Britain, June 1985.

Délery, J.M. and Marvin, J.G., "Shock-Wave Boundary Layer Interactions", AGARDograph No.280, NATO, 1986.

Délery, J.M., "A physical introduction to control techniques applied to turbulent separated flows", Fluids 2000, Denver, Colorado, AIAA-2000-2606, June 2000.

Délery, J.M. and Bur, R.S., "The physics of shock wave/boundary layer interaction control: last lessons learned", ECCOMAS 2000, Barcelona, Sept. 2000.

Doerffer P. and Szwaba, R. "Shock wave-boundary layer interaction control by streamwise vortices”, $21^{\text {th }}$ ICTAM Syposium, Warsaw, Poland, Aug. 2004. 
Dolling, D.S., "Unsteadiness of Shock-Induced Turbulent Separated Flows-Some Key Questions," $31^{\text {th }}$ AIAA Fluid Dynamics Conference \& Exhibit, AIAA 2001-2708, Anaheim, CA, June, 2001.

Dussauge, J.P., Dupont P. and Debiève, J.F.,"Unsteadiness in shock boundary layer interactions with separation", Aerospace Science and Technology, Vol . 10, pp. 85-91, 2006.

Dupont, P., Haddad, C. and Debiève, J.F., "Space and time organization in a shockinduced separated boundary layer", J. Fluid Mech., Vol. 559, pp.255-277, 2006.

Dupont, P. Piponniau, S., Sidorenko, A. and Debiève, J.F, "Investigation of an Oblique Shock Reflection with Separation”, AIAA Journal, Vol. 46, No. 6, pp.1365-1370, June 2008.

Edwards, J.R., "Numerical Simulations of Shock / Boundary Layer Interactions using Time-Dependent Modeling Techniques -a Survey of Recent Results," $46^{\text {th }}$ Aerospace Sciences Meeting \& Exhibit, Reno, NV, AIAA 2008-0525, Jan. 2008.

Elena, M. and Lacharme, J.P., "Experimental study of a supersonic turbulent boundary layer using a laser Doppler anemometer,” J. Theor. Appl. Mech. 7, pp. 175-190, 1988.

Elsinga, G.E., "Tomographic particle image velocimetry and its application to turbulent boundary layers," PhD thesis, Delft Technical University, The Netherlands, 2008.

Erengil, M.E. and Dolling, D.S., "Correlation of Separated Shock Motion with Pressure Fluctuations in the Incoming Boundary Layer, AIAA Journal, Vol. 29, No. 11, pp. 728-735, Apr. 1991.

Fernholz, H.H., Finley, P.J., "A critical compilation of turbulent boundary layer Data," AGARDDograph No. 223, 1977.

Freestone, M.M.,"Vortex Generators for control of shock-induced separation; Part 1: introduction for aerodynamics,"ESDU Data item 93024, 1995.

Ganapathisubramani, B., Longmire, E. K. and Marusic, I.,"Characteristics of vortex packets in turbulent boundary layers," J. Fluid Mech., Vol. 478, pp. 35-46, 2003.

Ganapathisubramani, B. Clemens, N.T. and Dolling D.S., "Large-scale motions in a supersonic boundary layer", J. Fluid mech.., Vol. 556, pp.271-282, 2006.

Ganapathisubramani , B. Clemens, N.T. and Dolling D.S., "Effects of upstream coherent structures on low-frequency motion of shock-induced turbulent separation", J. Fluid mech.., Vol. 585, pp.369-394, 2007.

Gefroh, D, Loth, E., Dutton, C and Mcllwain, S., "Control of an oblique shock/ boundary-layer interaction with airoelastic mesoflaps," AIAA Journal, Vol. 40, No. 12, pp. 2456-2466, Dec. 2002.

Green, J.E., "Interactions between shock waves and turbulent boundary layers. Prog. Aero. Sci., 11, pp. 253-340, 1970. 
Green, J.E., "Reflexion of an oblique shock wave by a turbulent boundary layer," J. Fluid Mech., Vol. 40, part 1, pp 81-95, 1970.

Hutchins, N. and Marusic, I.,"Evidence of very long meandering features in the logarithmic region of turbulent boundary layers," J. Fluid Mech., Vol. 579, pp. 1-28, 2007.

Hunt, D. and Nixon, D., "A Very Large Eddy Simulation of an Unsteady Shock Wave/Turbulent Boundary Layer Interaction,” AIAA-paper 95-2212, 1995.

Holden, H. and Babinsky, H., "Separated shock-boundary-layer interaction control using 3D devices,” AIAA paper 2003-0447, 2003.

Holden, H. and Babinsky, H., "Separated shock-boundary-layer interaction control using streamwise slots,” Journal of Aircraft, Vol. 42, No. 1, pp. 166-171, Jan.-Feb. 2005.

Holden, H. and Babinsky, H., "Effect of microvortex generators on separated normal shock/boundary layer interactions," Journal of Aircraft, Vol. 44, No. 1, pp. 170-174, Jan.-Feb. 2007.

Hou, Y.X., "Particle image velocimetry study of shock-induced turbulent boundary layer separation," $\mathrm{PhD}$ Thesis, Department of Aerospace Engineering and Engineering Mechanics. The University of Texas at Austin, 2003.

Humble, R.A., Scarano, F. and van Oudheusden, B.W., "Particle image velocimetry measurements of a shock wave/turbulent boundary layer interaction," Exp. Fluids, 43, pp. 173-183, Apr. 2007.

Humble, R.A., Elsinga, G.E., Scarano, F. and van Oudheusden, B.W., "Threedimensional unsteady flow organization of a shock wave/turbulent boundary layer interaction," J. Fluid mech., in print, 2008.

Humble, R.A. "Unsteady flow organization of a shock wave boundary layer interaction, "PhD thesis, Delft Technical University, The Netherlands, 2008.

Kim, K.C. and Adrian, R.J.,"Very large-scale motion in the outer layer," Phys. Fluids 11, pp. 417-422, 1999.

Klebanoff, P., "Characteristics of turbulence in a boundary layer with zero pressure gradient,” NACA Rept. 1247, 1955.

Lee, S., Loth, E. and Kim S., "LES of Supersonic Turbulent Boundary Layers with $\mu$ VGs," $25^{\text {th }}$ AIAA Applied Aerodynamics Conference, AIAA 2007-3916, Miami, FL, June, 2007.

Lin J.C., "Review of Research on Low-Profile Vortex Generators to Control Boundary-Layer Separation," Progress in Aerospace Sciences, Vol. 38, No. 4-5, pp. 389-420, May-July 2002. 
McClure, W.B., "An experimental study of the driving mechanism and control of the unsteady shock induced turbulent separation in a Mach 5 compression corner flow," $\mathrm{PhD}$ thesis, Department of Aerospace Engineering and Engineering Mechanics, The University of Texas at Austin, Texas, USA, 1992.

McCormick, D.C., "Shock/Boundary-Layer Interaction Control with Vortex Generators and Passive Cavity”, AAIA Journal, Vol. 31, No. 1, pp. 91-96, Jan. 1993.

Melling, A.,"Seeding gas flows for laser anemometry," In: Proceedings on the conference of advanced instrumentation for aero engine components. AGARD CP-399, 8.1, 1986.

Mounts, J.S. and Barber, T.J.,"Numerical Analysis of Shock-Induced Separation Allevation Using Vortex Generators," AIAA 1992-0751, Jan, 1992.

Peake, D.J., "The use of air injection to prevent separation of the turbulent boundary layer in supersonic flows,"ARC. P.No. 890, 1966.

Pearcey H.H., "Shock-Induced Separation and its Prevention by Boundary Layer Control," In Lachmann G.V. (editor), "Boundary Layer and Flow Control: Its principles and Applications (2 Volumes) Chapter 2 pp. 1170-1135. Pergamon Press. Oxford, 1961.

Pirozzoli, S. and Grasso, F., "Direct numerical simulation of impinging shock wave/turbulent boundary layer interaction at M=2.25,"Phys. Fluids, 18, 065113, 2006.

Pitt Ford, C.W. and Babinsky, H., "Micro-Ramp Control for Oblique Shock Wave/Boundary Layer Interactions," 37th AIAA Fluid Dynamics Conference and Exhibit, AIAA 2007-4115, Miami, FL, June 2007.

Plotkin, K.J., "Shock wave oscillation driven by turbulent boundary layer fluctuations," AIAA Journal, Vol. 13, pp. 1036-1040, 1975.

Poggie, J. and Smits, A.J., "Shock unsteadiness in a reattaching shear layer,"J. Fluid Mech., Vol. 429, pp. 155-185, 2001.

Raffel, M., Willert, C. and Kompenhans, J. ,'Particle image velocimetry: a practical guide," Springer, Heidelberg, 1998.

Raghunathan, S.,"Passive control of shock-boundary layer interaction," Prog. Aero. Sci., Vol. 25, pp 271-296, 1988.

Ringuette, M. J., Wu, M. and Martin, M. P., "Coherent Structures in Direct Numerical Simulation of Turbulent Boundary Layers at Mach 3," J. Fluid Mech., Vol. 594, pp. 59-69, 2008.

Scarano F., and Van Oudheusden B.W.,"Planar velocity measurements of a twodimensional compressible wake flow," Exp. Fluids, 34, pp. 430-441, 2003.

Schrijer, F.F.J., Scarano F., and Van Oudheusden B.W., "Application of PIV in a Mach 7 double-ramp flow," Exp. Fluids, 41, pp. 353-363, 2006. 
Schrijer, F.F.J., Scarano F.,"Particle slip compensation in steady compressible flows," $7^{\text {th }}$ international symposium on particle image velocimetry, Rome, Italy, Sep. 2007.

Selig, M.S., Andreopoulos, J., Muck, K.C., Dussauge, J.P. and Smits, A.J., "Turbulence structure in a shock wave/turbulent boundary-layer interaction," AAIA Journal, Vol. 27, pp. 862-869, 1989.

Simpson, R.L., "A review of some phenomena in turbulent flow separation," Journal of Fluids Engineering, 102, pp. 520-533, 1981.

Smith, F.T., "Theoretical prediction and design for vortex generators in turbulent boundary layers", J. Fluid Mech., Vol. 270, pp.91-131, 1994.

Smith, A. N., Holden, H. A., Babinsky, H., Ashill, P., and Fulker, J., "Control of Normal Shock Wave/Turbulent Boundary Layer InteractionsUsing Streamwise rooves,"AIAA paper, Jan. 2002.

Smits, A.J. and Dussauge, J.P., “Turbulent Shear Layers in Supersonic Flow”, second edition, AIP Press, 2006.

Souverein, L., Van Oudheusden, B.W., Scarano, F. and Dupont, P., "Unsteadiness characterization in a shock-wave turbulent boundary layer interaction through dual-PIV," $38^{\text {th }}$ Fluid Dynamics Conference and Exhibit, AIAA-2008-4169, Seattle, USA, June 2008.

Spina, E.F., "Organized structures in a supersonic turbulent boundary layer," $\mathrm{PhD}$ thesis, Department of Mechanical and Aerospace Engineering, Princeton University, NJ, USA, 1988.

Thomas, F.O., Putman, C.M. and Chu, H.C., "On the mechanism of unsteady shock oscillation in shock wave/turbulent boundary layer interactions", Experiments in Fluids, vol. 18, pp 69-81, Springer-Verlag, July 1994.

Tomkins, C.D. and Adrian, R.J., "Spanwise structure and scale growth in turbulent boundary Layers,” J. Fluid Mech., Vol. 490, pp. 37-74, 2003.

Tuinstra, M., “A PIV Investigation on Shock Wave Boundary Layer Interaction, An oblique shock impinging on a flat plate turbulent boundary layer", MSc. Thesis, Delft University of Technology, Aug. 2005.

Tufo, H.M., Fischer, P.F., Papka, M.E. and Szymansky, M, "Hairpin vortex formation, a case study for unsteady visualization," 41st CUG Conference, 1999.

Ünalmis, Ö.H. and Dolling, D.S., "Experimental Study of Causes of Unsteadiness of Shock-Induced Turbulent Separation," AAIA Journal, Vol. 36, No. 3, pp. 371-378, Mar. 1998.

Wu, M. and Martin, M.P., "Analysis of shock motion in shockwave/turbulent boundary layer interaction using direct numerical simulation data,'J. Fluid. Mech., Vol. 594, pp. 71-83, 2008. 
EFFECTS OF MICRO-RAMPS ON A SHOCK WAVE BOUNDARY LAYER INTERACTION 